\title{
INTERNET-DELIVERED COGNITIVE PROCESSING THERAPY FOR INDIVIDUALS WITH A HISTORY OF BULLYING VICTIMIZATION: A RANDOMIZED CONTROLLED TRIAL
}

\author{
A Thesis \\ Submitted to the Faculty of Graduate Studies and Research in Partial Fulfillment of the \\ Requirements for the Degree of \\ Doctor of Philosophy \\ in \\ Clinical Psychology \\ University of Regina \\ By \\ Audur Sjöfn Thorisdottir \\ Regina, Saskatchewan \\ September, 2019
}

Copyright 2019: A.S. Thorisdottir 


\section{UNIVERSITY OF REGINA}

\section{FACULTY OF GRADUATE STUDIES AND RESEARCH SUPERVISORY AND EXAMINING COMMITTEE}

Audur Sjöfn Thorisdottir, candidate for the degree of Doctor of Philosophy in Clinical Psychology, has presented a thesis titled, Internet-Delivered Cognitive Processing Therapy for Individuals with a History of Bullying Victimization: A Randomized Controlled Trial, in an oral examination held on September 10, 2019. The following committee members have found the thesis acceptable in form and content, and that the candidate demonstrated satisfactory knowledge of the subject material.

External Examiner: $\quad$ Dr. Laura Dixon, University of Mississippi ${ }^{\star}$

Supervisor: $\quad$ Dr. Gordon Asmundson, Department of Psychology

Committee Member: $\quad$ Dr. Heather Hadjistavropoulos, Department of Psychology

Committee Member: $\quad$ Dr. Kristi Wright, Department of Psychology**

Committee Member: $\quad$ Dr. Ronald Martin, Faculty of Education

Chair of Defense: $\quad$ Dr. Nader Mobed, Department of Physics

*Present via Skype

${ }^{* *}$ Not present at defense 


\begin{abstract}
Bullying victimization is directly associated with a wide array of longstanding psychological problems (e.g., Takizawa Maughan, \& Arseneault, 2014; Vaillancourt et al., 2013). Nevertheless, few comprehensive victim-tailored interventions exist that explicitly target the negative impact of bullying. Evidence from both neurobiological and psychological research suggests that victims experience bullying as trauma; therefore, treating the distress associated with bullying victimization using trauma-focused intervention may be beneficial. The purposes of this randomized controlled trial were to: (a) test an internet-delivered, therapist-guided (i.e., therapist monitored client progress through the treatment modules and provided feedback and support through secure e-mail messaging) and content-modified (i.e., to fit the experience of bullying victimization) version of cognitive processing therapy (CPT) for the psychological distress associated with bullying victimization, and (b) to examine risk and protective factors in the pathways from bullying victimization to psychological adjustment. The sample comprised 52 adults who self-identified as victims of bullying. Participants were randomized into internet-delivered CPT, an active control group of internet-delivered stress management $(\mathrm{SM})$, or a waitlist. Treatment outcomes included maladaptive appraisals, symptoms of posttraumatic stress disorder (PTSD), depression, anxiety, anger, and related latent constructs (e.g., distress tolerance). Secondary measures examining risk and protective factors included assessment of coping styles, social support, and resilience. Treatment outcome and secondary measures were administered pre-treatment, posttreatment, and at one-month follow-up. Symptoms of depression, anxiety, and anger were, further, monitored with weekly measures. Hierarchical linear modeling and multiple
\end{abstract}


regressions were used to analyse the data. Results indicated that CPT was effective in reducing maladaptive self-appraisals related to bullying victimization and symptoms of PTSD compared to the waitlist and SM, whereas SM outperformed CPT and the waitlist in reducing general psychological distress. Event centrality (i.e., the extent to which an experience has become central to identity and understanding of the world) led to stronger maladaptive appraisals for participants with a tendency to engage in catastrophizing. Number of reported lifetime bullying victimization experiences and anger also predicted stronger maladaptive appraisals. The overall findings of this trial suggest that internetdelivered CPT is effective for the psychological distress and maladaptive appraisals associated with bullying victimization, but adaptions might be needed to target more effectively symptoms of general distress and latent constructs related to anxiety and depression. 


\section{ACKNOWLEDGEMENTS}

I would like to acknowledge and express my sincere gratitude to several

individuals who were instrumental to my success in completing this challenging, yet rewarding project. I thank my supervisor Dr. Gordon Asmundson for his encouragement, guidance, and wisdom through the years. I thank my distinguished committee members, Drs. Heather Hadjistavropoulos, Kristi Wright, and Ronald E. Martin for their insightful and excellent feedback throughout this process. I thank my fellow past and present AIBL members for their support and feedback and for making every day better in the lab. I thank my dear cohort, Michelle, Andreea, Mel, and Jill, for their friendship, advice and support throughout my doctoral studies and this dissertation. I thank my partner, Aaron, for his support in getting me to the finish line. Finally, I sincerely thank the people who participated in this study, who inspired me by their openness and willingness to work with me through their painful bullying victimization experiences. 


\title{
DEDICATION
}

\author{
Ég tileinka pessari doktorsritgerð foreldrum mínum, Jónínu og Póri. \\ Kærar pakkir fyrir stuðningin í gegnum árin og fyrir að kenna mér að trúa á sjálfa \\ mig og fylgja draumum mínum.
}

[I dedicate this dissertation to my parents, Jónína and Pórir. Thank you for your support throughout the years and for teaching me to believe in myself and follow my dreams.] 


\section{TABLE OF CONTENTS}

ABSTRACT. i

ACKNOWLEDGEMENTS .............................................................................................................. iii

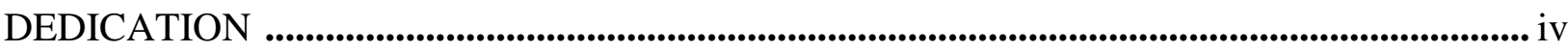

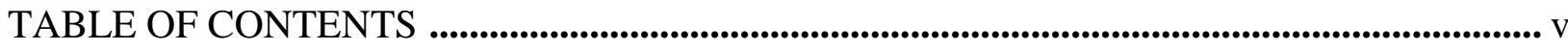

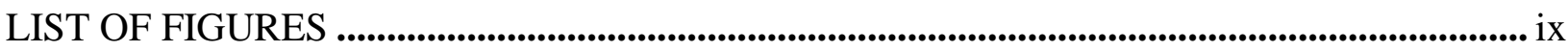

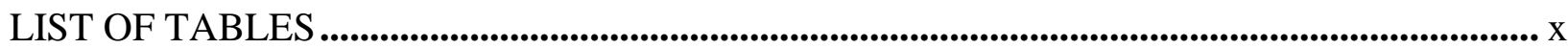

LIST OF APPENDICES ......................................................................................................................................... xii

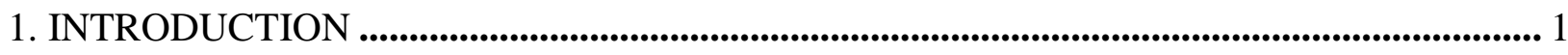

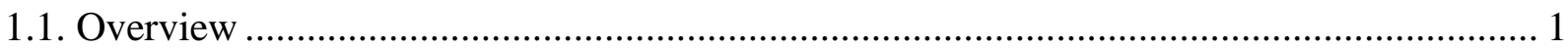

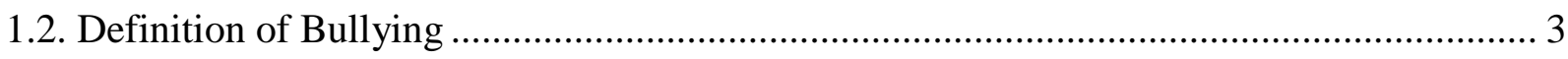

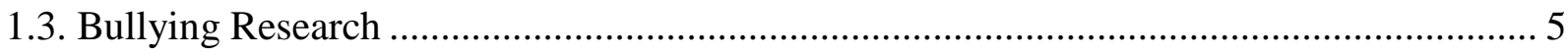

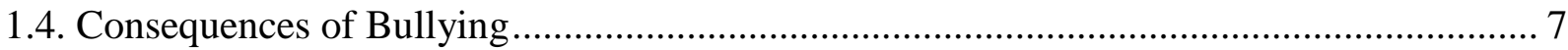

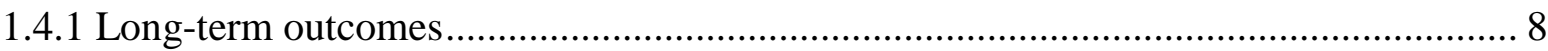

1.5. Bullying Prevention and Intervention Programs....................................................... 10

1.6. Pathways from Bullying to Long-term Outcomes .................................................. 11

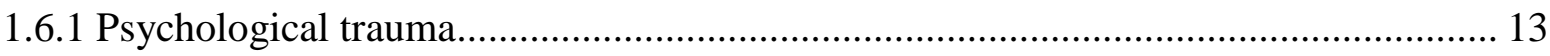

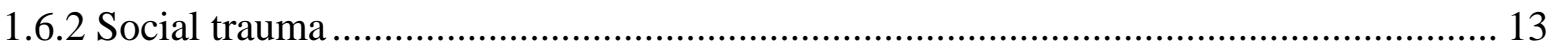

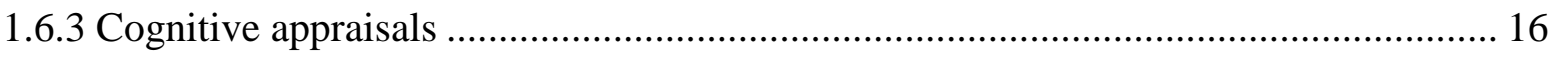

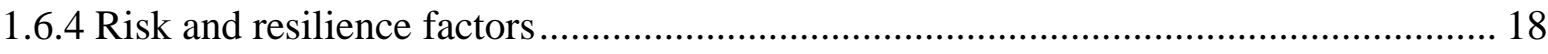

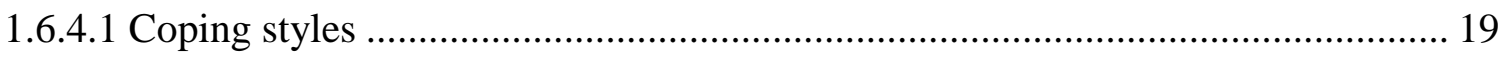

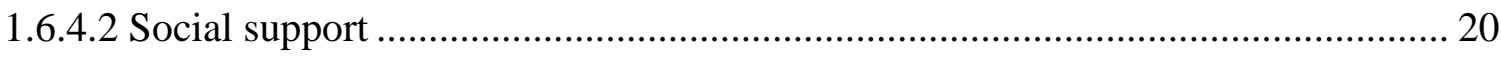

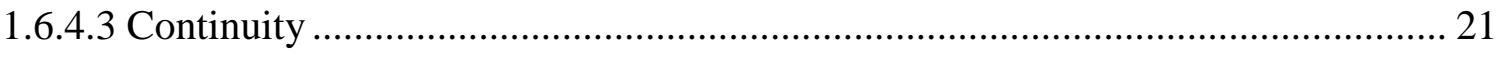

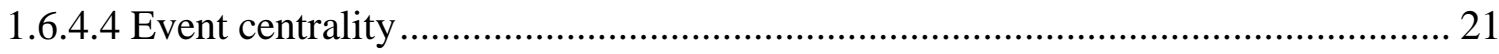

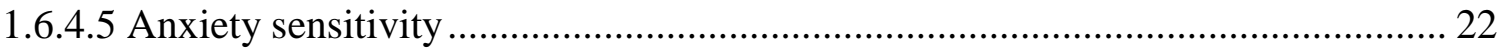

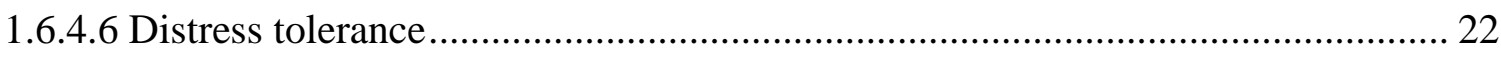

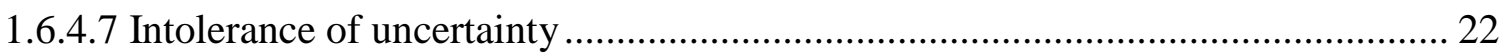




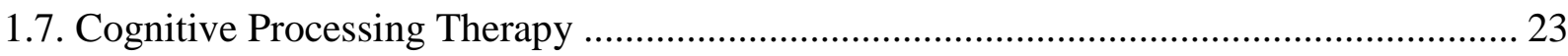

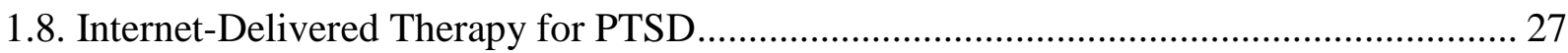

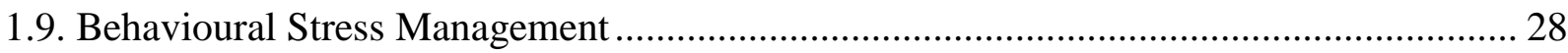

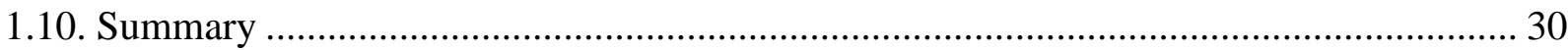

2. CURRENT INVESTIGATION AND METHODS ........................................................................... 32

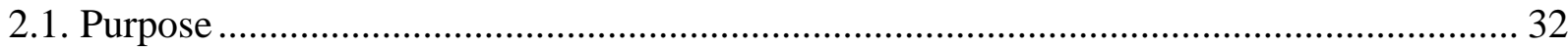

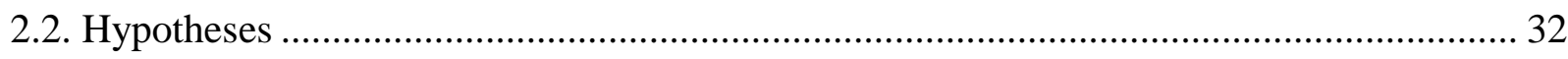

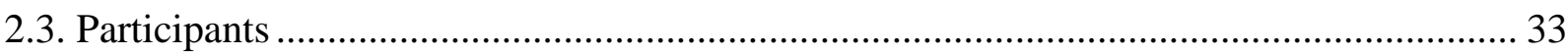

2.4. Acceptability Testing ................................................................................................... 34

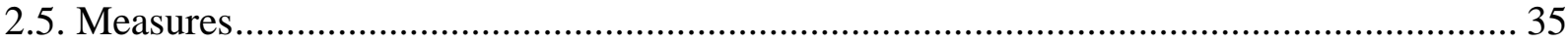

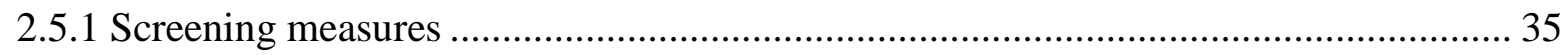

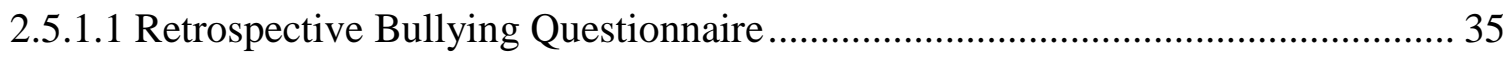

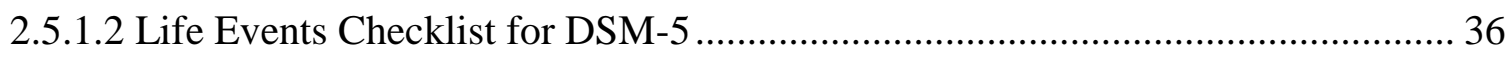

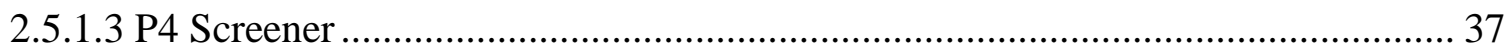

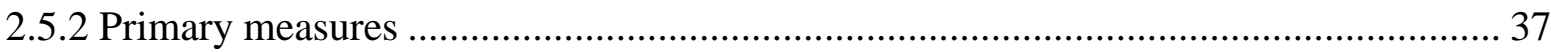

2.5.2.1 The Posttraumatic Cognitions Inventory .............................................................. 37

2.5.2.2 Posttraumatic Stress Disorder Checklist for DSM-5 ............................................ 38

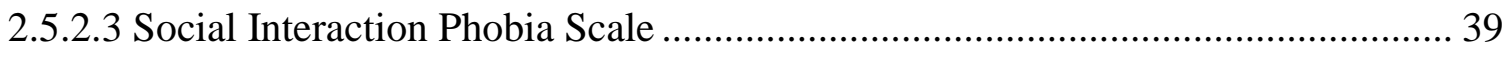

2.5.2.4 Depression Anxiety Stress Scale-21 …………………………...................... 39

2.5.2.5 Dimensions of Anger Reactions-5 Scale .............................................................. 40

2.5.2.6 Distress Tolerance Scale................................................................................... 40

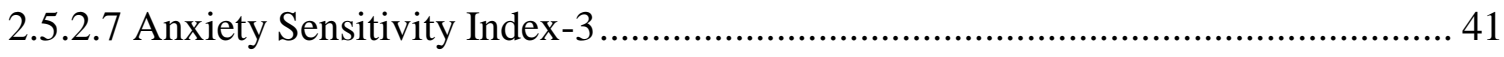

2.5.2.8 Intolerance of Uncertainty Scale-short form ..................................................... 41

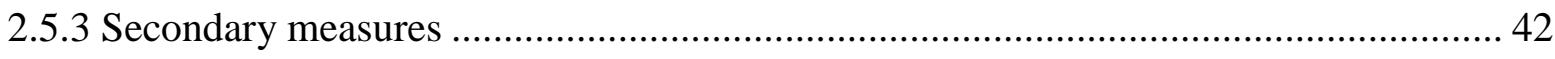

2.5.2.1 The Centrality of Event Scale-short ………….................................................. 42

2.5.3.2 Cognitive Emotion Regulation Questionnaire-short ............................................ 42

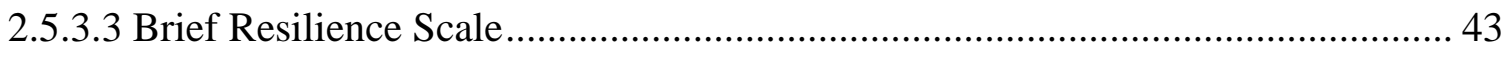


2.5.3.4 Multidimensional Scale of Perceived Social Support .......................................... 44

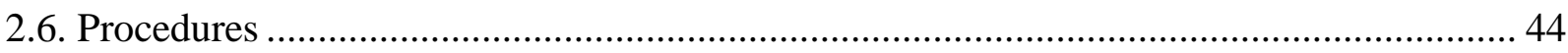

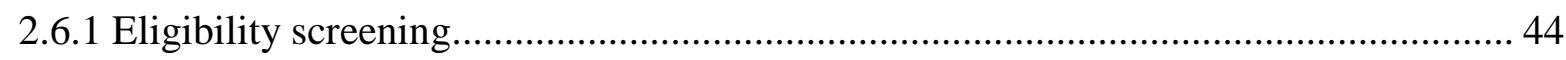

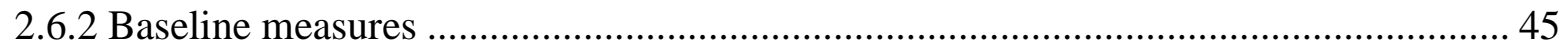

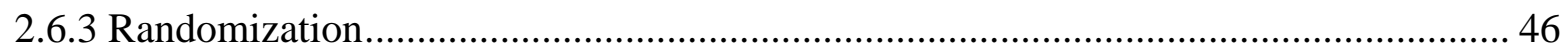

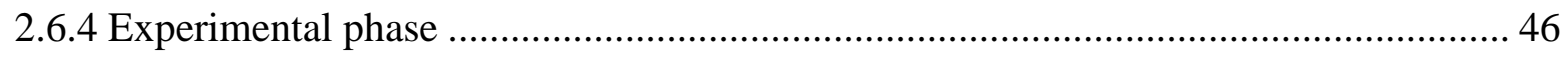

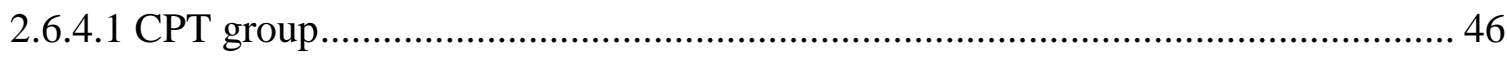

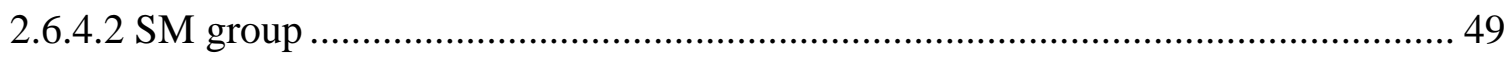

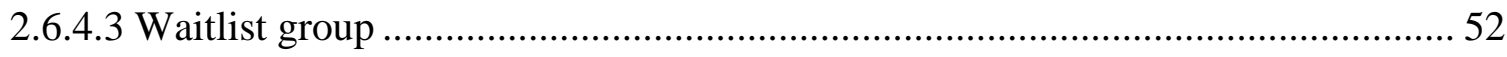

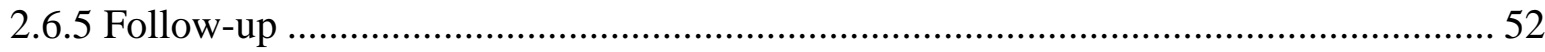

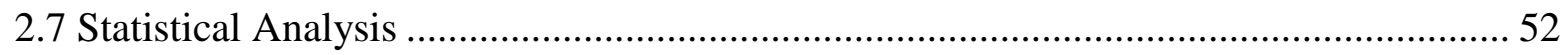

3. RESULTS ............................................................................................................................... 56

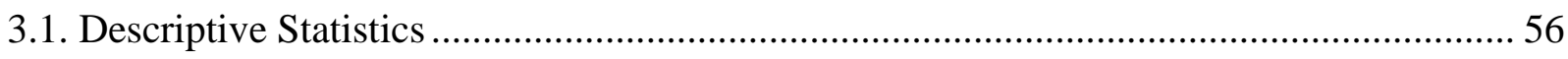

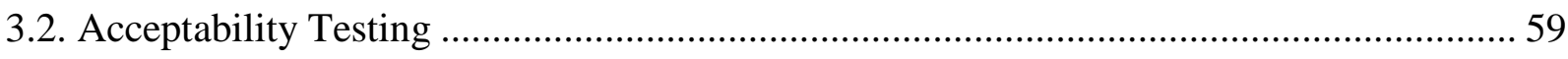

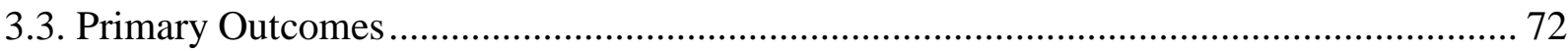

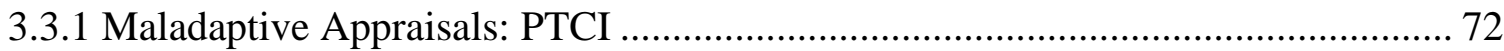

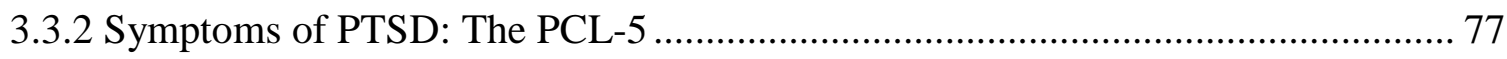

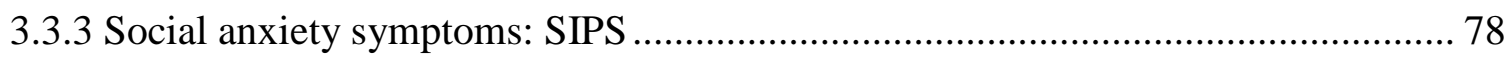

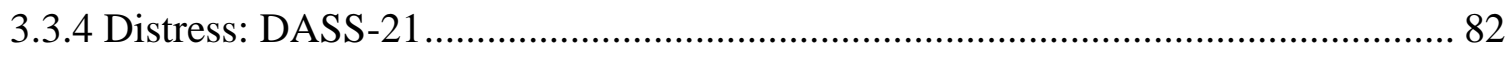

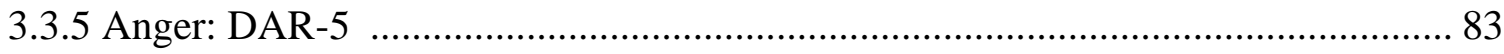

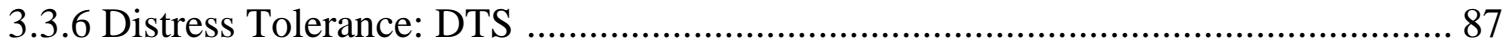

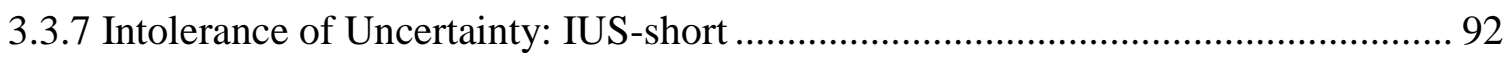

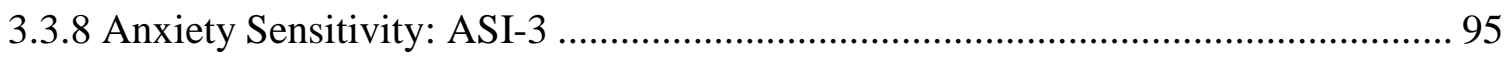

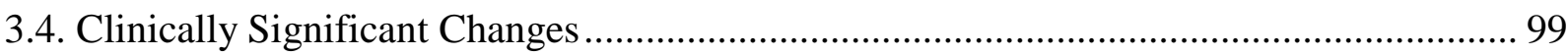

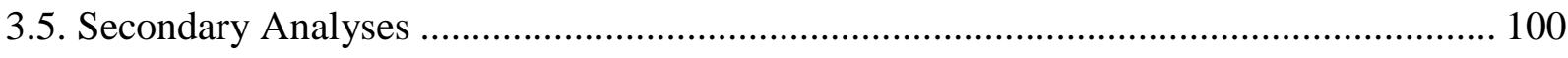

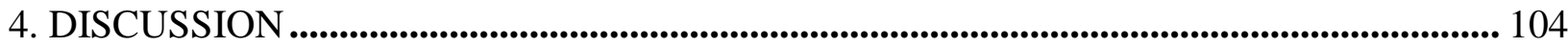

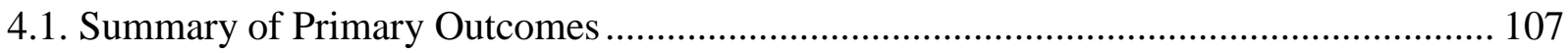




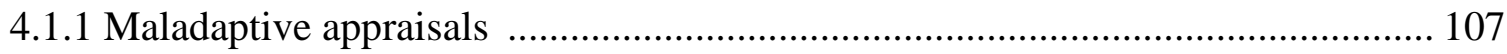

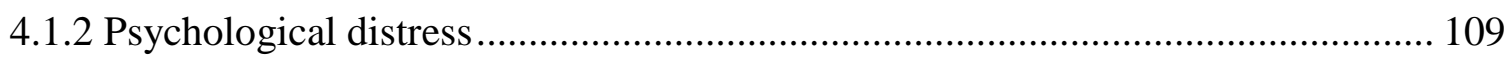

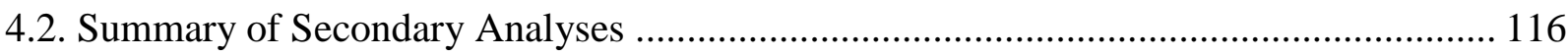

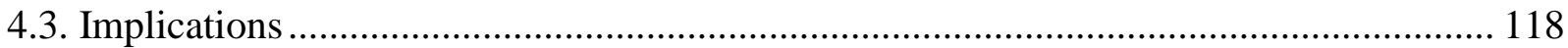

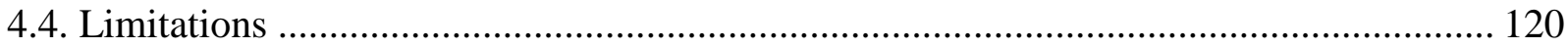

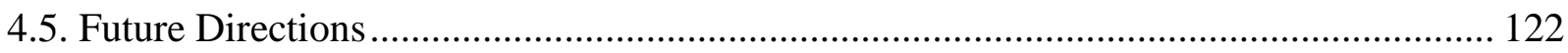

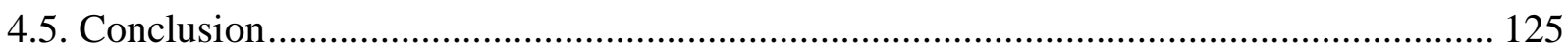

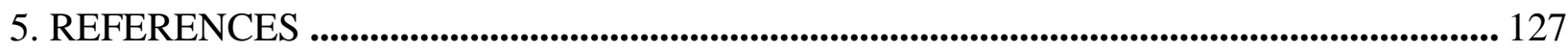

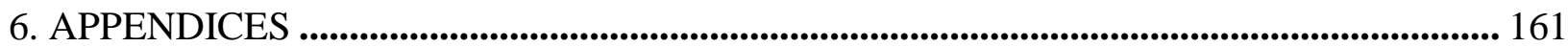




\section{LIST OF FIGURES}

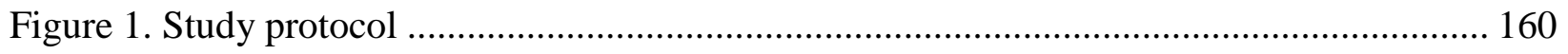

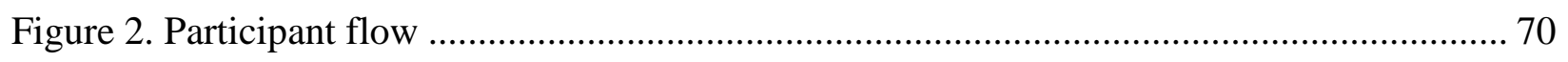

Figure 3. Session-by-session changes in SIPS, DASS-21, and DAR-5 scores

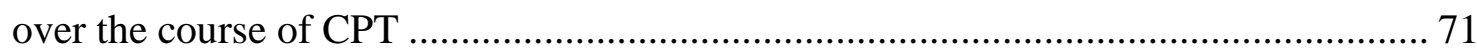

Figure 4. Session-by-session changes in SIPS, DASS-21, and DAR-5 scores

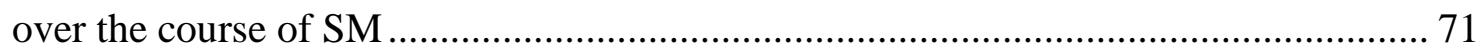

Figure 5. Mediation effect of catastrophizing on the relationship between event

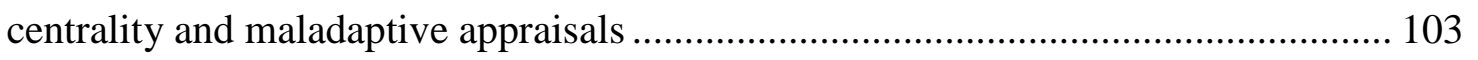

Figure 6. Moderation effect of anger on the relationship between number of bullying victimization experiences and maladaptive appraisals ........................................... 103 


\section{LIST OF TABLES}

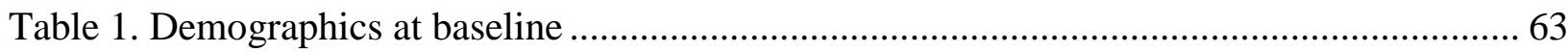

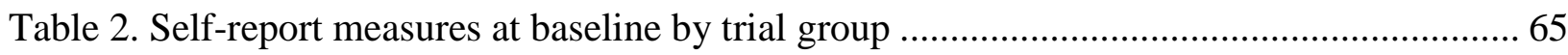

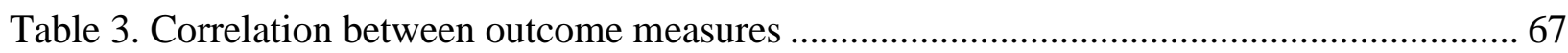

Table 4. Overview of bullying victimization experiences

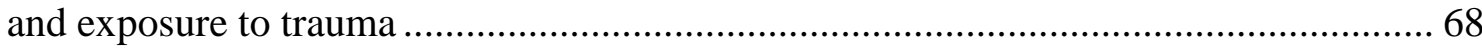

Table 5. Multilevel model of total PTCI scores over time ………………………………............ 75

Table 6. Multilevel model of PTCI Negative Self-Beliefs over time ………………………..... 75

Table 7. Multilevel model of PTCI Negative Other-Beliefs over time ……………………........ 76

Table 8. Multilevel model of PTCI Self-Blame Beliefs over time .................................................. 76

Table 9. Multilevel model of PCL-5 scores over time............................................................... 77

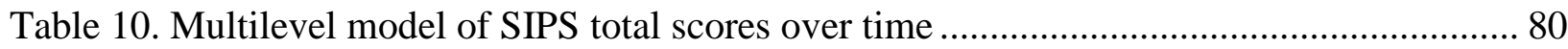

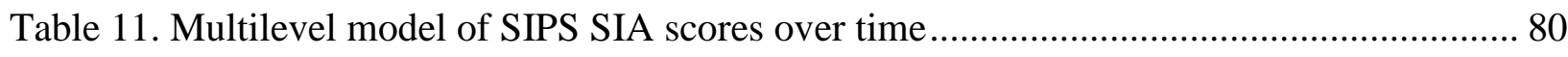

Table 12. Multilevel model of SIPS FOE scores over time..................................................... 81

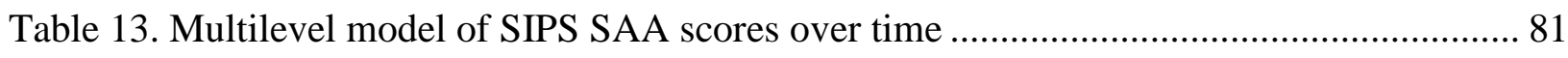

Table 14. Multilevel model of DASS-21 total scores over time..................................................... 84

Table 15. Multilevel model of DASS-21 Depression scores over time.......................................... 84

Table 16. Multilevel model of DASS-21 Anxiety scores over time............................................ 85

Table 17. Multilevel model of DASS-21 Stress scores over time …………………………........ 85

Table 18. Multilevel model of DAR-5 scores over time ............................................................ 86

Table 19. Multilevel model of DTS total scores over time........................................................... 89

Table 20. Multilevel model of DTS Absorption scores over time .................................................. 89

Table 21. Multilevel model of DTS Appraisal scores over time ……………………….............. 90

Table 22. Multilevel model of DTS Regulation scores over time …………………................... 90

Table 23. Multilevel model of DTS Tolerance scores over time .................................................. 91

Table 24. Multilevel model of IUS-12 total scores over time ……............................................... 93

Table 25. Multilevel model of IUS-12 Inhibitory scores over time ............................................. 93 
Table 26. Multilevel model of IUS-12 Prospective scores over time 94

Table 27. Multilevel model of ASI-3 total scores over time .................................................. 97

Table 28. Multilevel model of ASI-3 Physical scores over time .......................................... 97

Table 29. Multilevel model of ASI-3 Cognitive scores over time........................................... 98

Table 30. Multilevel model of ASI-3 Social scores over time ............................................. 98 


\section{LIST OF APPENDICES}

Appendix A: Certificate of Research Approval _..................................................................... 162

Appendix B: Renewal of Research Approval Certificate ………………………………........ 162

Appendix C: Certificate of Approved Amendment to Research Approval .................................. 163

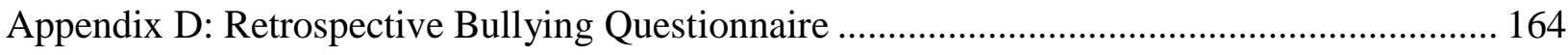

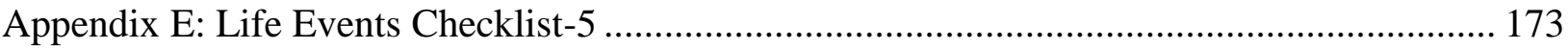

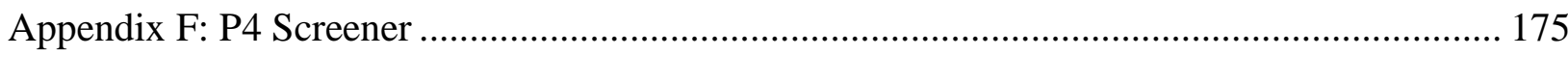

Appendix G: Posttraumatic Stress Disorder Checklist for DSM-5 …….................................. 176

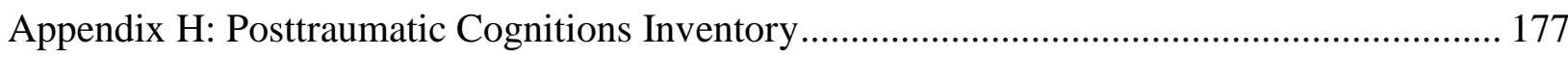

Appendix I: Social Interaction Phobia Scale ........................................................................ 179

Appendix J: Depression Anxiety Stress Scale-21 ................................................................. 180

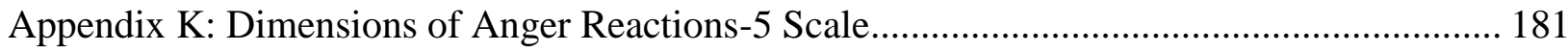

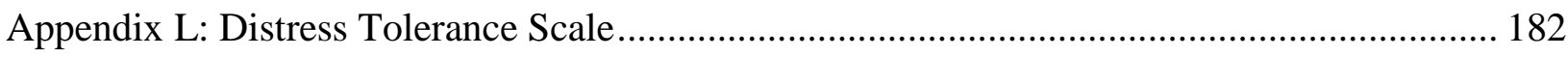

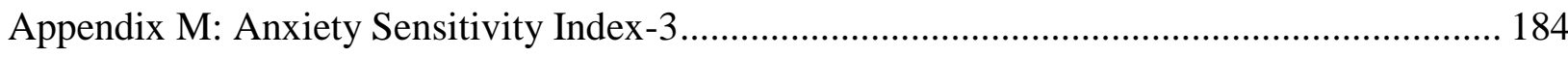

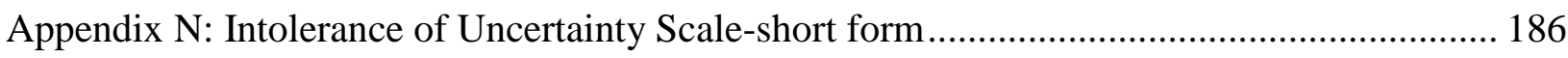

Appendix O: Centrality of Event Scale ………………….................................................... 187

Appendix P: Cognitive Emotion Regulation Questionnaire-short............................................... 188

Appendix Q: Brief Resilience Scale ...................................................................................... 189

Appendix R: Multidimensional Scale of Perceived Social Support ........................................... 190

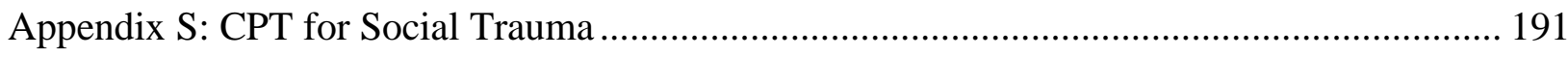

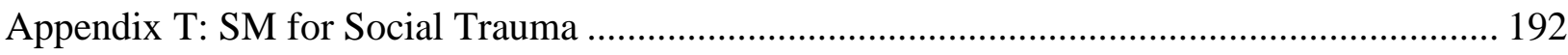

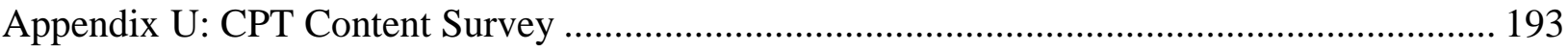

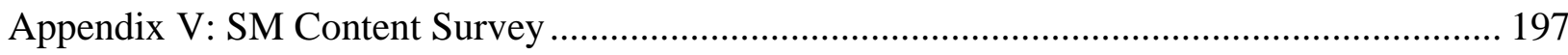




\section{INTRODUCTION}

\subsection{Overview}

A large body of research on the consequences of bullying demonstrates the pervasive negative effects of victimization (e.g., Gibb, Horwood \& Fergusson, 2011; Nansel, Craig, Overpeck, Saluja, \& Ruan, 2004). Victims of bullying are at higher risk for various health and adjustment difficulties (e.g., symptoms of anxiety and depression, unstable interpersonal relationships) that may persist long after the aggressions have ceased (Klomek et al., 2009; Kumpulainen \& Räsänen, 2000; Takizawa, Maughan, \& Arseneault, 2014). Nevertheless, few specific and comprehensive victim-tailored interventions exist that systematically focus on reducing the negative psychological impact of bullying victimization (Ttofi \& Farrington, 2011).

Bullying victimization is in many ways analogous to trauma. Trauma refers to psychological responses to experiences that pose a risk of serious injury, sexual violence, or actual or threatened death (American Psychiatric Association, 2013). Although threats of serious injury and death may be insinuated by physical and verbal bullying behaviours, other more subtle and non-life threatening forms of bullying may be equally traumatizing (see for example, Carleton, Peluso, Collimore, \& Asmundson, 2011; Mol et al., 2005). Indeed, both direct (e.g., physical aggressions) and indirect (e.g., social manipulation) forms of bullying victimization can lead to symptoms of posttraumatic stress (Erwin, Heimberg, Marx, \& Franklin, 2006; Nielsen, Tangen, Idsoe, Matthiesen, \& Magerøy, 2015). The parallels between bullying victimization and trauma are further supported by findings from neurobiological studies suggesting that bullying leads to the same longlasting dysregulations in stress hormones (e.g., cortisol) as various forms of life- 
threatening childhood trauma (Vaillancourt, Hymel, \& McDougall, 2013). By framing bullying victimization as trauma, it follows that trauma-focused treatments may be beneficial for alleviating the psychological distress stemming from exposure to such victimization.

Cognitive processing therapy (CPT) may be a viable option for treating the negative psychological impact of bullying victimization. CPT is based on socialcognitive theories which emphasize the role of maladaptive trauma appraisals in the onset and maintenance of posttraumatic stress disorder (PTSD). CPT focuses on identifying and updating dysfunctional beliefs about the causes and consequences of trauma that hinder recovery from posttraumatic stress (Resick et al., 2002; Resick, Monson, \& Chard, 2014). Maladaptive appraisals about bullying may be an important target in treatment. Numerous studies suggest that bullying victimization leads to negative self-perceptions in terms of worth, competency, and esteem (Boulton, Smith, \& Cowie, 2010; McDougall \& Vaillancourt, 2015). Negative interpretations about the bullying experience have also been found to predict the persistence of psychological distress following victimization (Taylor, Sullivan, \& Kliewer, 2013). Therefore, a treatment focused on identifying and updating maladaptive bullying appraisals is warranted. Developing a treatment for bullying victimization that can be delivered through the internet may be advantageous for increased client accessibility (e.g., clients living in rural areas that have to travel long distances to access mental health services, clients with restricted mobility, flexibility of accessing treatment content), affordability, and acceptance (e.g., fear of stigmatization; Sijbrandij, Kunovski, \& Cuijpers, 2016). 
This dissertation is structured as follows. First, an overview of bullying research is provided. This overview includes details on prevalence rates, outcomes associated with victimization, and bullying prevention and intervention efforts. Second, research evidence linking bullying victimization to trauma, cognitive theories of PTSD, research evidence for CPT, internet-delivered psychotherapies and stress management techniques for PTSD, as well as risk and resilience factors in the relationship between trauma and psychological distress are discussed. Third, an overview of the methodology of the trial is presented. Finally, the results, implications, and limitations of the trial are discussed.

\subsection{Definition of Bullying}

Bullying, which comprises repeated acts performed by one or more individuals that are intended to harm or intimidate others less powerful (Hoertel, Strat, Lavaud, \& Limosin, 2012), is a prevalent social issue that negatively affects youth and adults worldwide (Carney \& Merell, 2001). It is a heterogeneous concept that describes a wide array of behaviours, including physical aggression (e.g., beating, destroying things), verbal aggression (e.g., teasing, insulting), and social manipulation (e.g., deliberately ignoring; Berger, 2007; Sourander, 2007). Bullying behaviours can be further conceptualized as direct or indirect. Direct bullying involves confrontation (e.g., physical aggression) whereas indirect bullying behaviours are covert and often involve a third party (e.g., spreading rumours; Espelage \& Swearer, 2003). Although the manifestation of bullying can include one or more types of behaviour, certain behaviours are usually more prevalent during different developmental periods. Specifically, physical aggressions are more prevalent among younger children and decline with age, whereas behaviours of verbal aggression and social manipulation increase with age (Berger, 2007). Furthermore, 
the genders differ in the type of bullying behavior they engage in; specifically, boys tend to engage in more physical aggressions than girls, and girls tend to engage in social manipulation behaviours at younger ages than boys (Graham, 2016).

Over the past decade, a new form of bullying that occurs through cyber space has emerged. Cyber-bullying differs from other types of bullying as it is not confined to social settings, such as schools, but can occur anytime and anywhere with electronic devices. Furthermore, through the virtual environment of cyber space, the communication is faster, more widespread, and often anonymous. These conditions may create disinhibition, as there is limited accountability for an individual's actions, which can lead to more severe aggressions (Antoniadou \& Kokkinos, 2015; Sari \& Camadan, 2016).

Despite its heterogeneous nature, consensus exists in the research literature on three conditions that differentiate bullying from other aggressive behaviours. First, there is a power differential between the victim and bullies that make it difficult for the victim to defend themselves. Second, the behaviours (i.e., verbal, physical, or social manipulations) are harmful to the victim in some way. Third, the behaviors occur repeatedly over extended time (Berger, 2007; Olweus, 2001; Salin et al., 2019). For example, spreading rumors for months about a new student in the class to make others dislike him or her would be considered bullying. Conflict at work that escalates over time to humiliation in front of co-workers and isolation would also be considered bullying. In contrast, gentle teasing on a few isolated occasions would typically not be considered bullying (Olweus, 2001; Notalaers et al., 2018; Salin et al., 2019). 


\subsection{Bullying Research}

Empirical research on bullying began in the late 1970s with the pioneering work of Olweus on school aggression in Scandinavia. Olweus's research gained attention when the Norwegian government sought his expertise in a national campaign against school bullying that was launched in 1982, following the tragic suicides of three adolescent victims of bullying (Allanson, Lester, \& Notar, 2015; Olweus, 1994). Academic interest in the subject began to arise soon after, and researchers around the world started studying bullying among children and adolescents (Berger, 2007).

Studies on school bullying for the past few decades demonstrate that it is a common problem; however, the prevalence of reported school bullying varies greatly by country. Nansel and colleagues (2004) found in a cross-national comparison that involvement in bullying for children and adolescents ranged from 9\% in Sweden to 54\% in Lithuania. Similarly, Due et al. (2005) found that across 28 countries, the prevalence of adolescent involvement in bullying was lowest in Sweden at $6.3 \%$ and highest in Lithuania at $41.4 \%$. However, the large difference in prevalence rates of bullying across countries may be a function of different cultural conceptualizations and understanding of the phenomena (Cook, Williams, Guerra, Kim, \& Sadek, 2010; Salin et al., 2018), as well as differences in the research methodology and sampling methods (Nielsen, Matthiesen, \& Einarsen, 2010). When it comes to prevalence across age, school bullying behaviours have been found to peak during middle-childhood and then decline with age; but, bullying is not confined to children and youth (Eslea \& Rees, 2001).

Bullying among adults in the workplace became a topic of research in the 1980s. Early efforts at studying the phenomena, which were inspired by the research on bullying 
in schools, confirmed that the same hallmark features of repetitiveness and unwanted negative behaviours applied to bullying in the workplace (Einarsen, Hoel, Zapf, \& Cooper, 2011). Since then, accumulating research suggests that the prevalence rates of bullying in adult samples at workplaces are high, varying from nine to $40 \%$ (Einarsen $\&$ Skogstad, 1996; Vega \& Comer, 2005).

Bullying perpetrators and victims have traditionally been categorized dichotomously in the research literature (Espelage et al., 2003). Perpetrators were the individuals responsible for the aggressive acts and victims the ones subjected to them. With more research, however, these binary categories have been challenged and scholars now stress looking at the social context in which the bullying occurs (Veenstra, Lindenberg, Oldehinkel, De Winter, \& Verhulst, 2003). Although one individual can usually be identified as the bully (i.e., that initiates and perpetrates the aggressive acts against the victim), research suggests that the peer group and colleagues at work are also partly responsible for the maintenance and escalation of bullying (Howard, Landau, \& Pryor, 2014; Olweus, 2001). Peers and colleagues are present during most bullying occurrences and may reinforce the bully's aggressions against the victim by actively participating in their behaviour or passively observing it (Einarsen et al., 2011; Hawkins, Pepler \& Craig, 2001). Furthermore, a subgroup of bullying victims also engages in bully perpetration (i.e., proactive victims/bully-victims; Veenstra et al., 2005). Research suggests that bully-victims are more disliked and rejected by their peers than bullies and passive victims (i.e., victims that do not bully others), and are at higher risk for more persistent and severe bullying than passive victims (Salmivalli \& Nieminen, 2002). 


\subsection{Consequences of Bullying}

Research over the past few decades has consistently found that bullying has detrimental consequences (e.g., Kumpulainen \& Räsänen, 2000; Sourander et al., 2009). Victims of bullying, whether passive victims or bully-victims, are at greater risk for various emotional problems, including anger, fear, anxiety, and depression (McDougall \& Vaillancourt, 2015), and for self-harm behaviors, suicidal ideation, and attempted suicide (Lereya et al., 2013). Victims often report feeling lonely and may avoid school or work, or have difficulties concentrating, which has associated detrimental consequences for performance and productivity (Einarsen et al., 2011; McDougall \& Vaillancourt, 2015; Nansel et al., 2004). Victimization is further linked to poorer physical health and health-risk behaviours, including somatic complaints, higher levels of sedentary behaviours, alcohol use, and smoking (Hertz, Jones, Barrios, David-Ferdon, \& Holt, 2015; Katapally, Thorisdottir, Laxer, Quian, \& Leatherdale, 2018; McDougall \& Vaillancourt, 2015). Furthermore, bullying perpetrators are themselves at increased risk of mental health and adjustment difficulties, including subsequent antisocial behaviors, substance abuse disorder, depression, and anxiety (McMillan, Thorisdottir, \& Asmundson, 2017; Shireen, Janapana, Rehmatullah, Temuri, \& Azim, 2014; Sourander et al., 2007).

Cross-national comparisons demonstrate that involvement in bullying is associated with negative health outcomes and maladjustment worldwide. Due et al. (2005) examined the association between bullying and mental and physical health in 123,227 adolescents $(11,13$, and 15 years old) in 28 countries. Despite varying levels of reported bullying and substantial cultural differences between the participating countries 
(e.g., participants from an Inuit culture in Greenland and participants from Israel), a consistent pattern was found. Victims of bullying had more negative psychological concerns (e.g., feeling low, irritable, helpless) and physical symptoms (e.g., stomach pains, dizziness) than their peers. Similarly, Nansel et al. (2004) found that adolescent bullies, victims, and bully-victims in 25 countries consistently reported higher levels of health problems and psychosocial and academic adjustment than uninvolved peers. Finally, Verkuil, Atasayi and Molendijk (2015) conducted a meta-analysis on the association between workplace bullying and mental health outcomes using crosssectional and longitudinal data from 63 studies in 19 countries. They found that workplace bullying predicted the development of depression, anxiety, symptoms of PTSD, and various stress-related psychological complaints, with effect sizes ranging from 0.15 - 0.51 (i.e., compared to non-involved workers). The combined findings of these studies underscore that bullying victimization universally leads to negative outcomes across all age groups.

1.4.1. Long-term outcomes of bullying. Prospective longitudinal studies suggest that the detrimental consequences of bullying may persist for years after the aggressions have ceased (Klomek et al., 2012; Mebane, 2010). Sourander et al. (2009) and Klomek et al. (2009) examined long-term mental health outcomes associated with bullying using data from a large Finnish birth-cohort study. Information about bullying and mental health was gathered when the participants were eight years old using self-report questionnaires, as well as teacher and parent-rated questionnaires. Mental health was subsequently re-evaluated using hospital records of prescribed psychiatric medications and psychiatric hospitalizations when the participants were 13 and 24 years old. Whereas 
psychiatric symptoms, attempted suicides, and completed suicides in boys that had been victims of bullying were dependent upon the baseline psychiatric disorder symptom measures, bullying victimization of girls predicted later psychiatric problems, suicide attempts, and completed suicides irrespective of the baseline measures. Male participants who had been victims as children were at a greater risk for a wide array of psychiatric outcomes (e.g., use of antidepressants and antipsychotic medication).

To address some of the limitations involved with relying on hospital records for psychiatric symptoms (e.g., underreporting), Copeland, Wolke, Angold, and Costello (2013) used self-report questionnaires to assess the long-term psychological outcomes of bullying involvement. They found that participants who had been bullied between nine and 16 years of age were more likely to meet diagnostic criteria for depression and anxiety disorders at 19, 21, or 24-26 years compared to participants who had not experienced bullying. Additionally, participants who had bullied others were more likely to meet diagnostic criteria for antisocial personality disorder.

To elucidate further the adult health variables associated with childhood bullying, Takizawa and colleagues (2014) assessed the psychiatric correlates of 7,771 individuals four decades after bullying victimization exposure and compared the outcome measures to those of non-victimized participants. Bullying and psychiatric symptoms were assessed with parental interviews and teacher rated scales when the participants were seven and 11 years old and symptoms of psychological disorders and psychological distress were reassessed with interviews and self-report questionnaires at ages 23, 45, and 50. Bullying victimization was associated with higher levels of psychological distress at all ages compared to non-victimized participants. Furthermore, participants who had been bullied 
frequently (i.e., identified by subjective parental assessment) when they were children were 1.95 times more likely to receive a diagnosis of depression, 1.65 times more likely to meet diagnostic criteria for an anxiety disorder, and 2.21 times more likely to report suicidality (i.e., suicidal ideation, suicide plans and attempts) as adults, independent of baseline measures of psychiatric symptoms and exposure to other forms of adversity early in life.

\subsection{Bullying prevention and intervention programs}

Bullying prevention and intervention programs at schools typically focus on bullies, victims, or both, or take a multifaceted approach that involves teachers, staff, peers, and in some instances the home environment of the involved youth. Anti-bullying efforts at workplaces usually fall into levels of policy (e.g., "no tolerance" policy), organization specific efforts (e.g., educated management about conflict resolution), and employer focused intervention (Vartia \& Leka, 2011). At each level, interventions can further be differentiated into stages, including prevention efforts (i.e., primary stage), intervention if bullying has occurred (i.e., secondary stage), and rehabilitation to reduce the negative impact of bullying on the individuals involved (i.e., tertiary stage; Leka et al., 2008). Programs at both schools and workplaces commonly teach bullying awareness, how to identify accumulating aggressions, and some form of social skills training (e.g., how to stop bullying, how to support the victim, improving peer relationships and workplace culture; Farrington \& Ttofi, 2009, Vartia \& Leka, 2011).

Despite bullying interventions being in place at schools and workplaces, problems with implementation frequently arise (Merrell, Gueldner, Ross, \& Isava, 2008; Ttofi \& Farrington, 2011). The effectiveness of bullying intervention at workplaces is directly 
related to the commitment of supervisors to successfully implement and follow through with the intervention procedures; however, in many cases the perpetrator of the bullying victimization experience in the workplace is the supervisor (Einarsen, Zapf, \& Cooper, 2011; Leka et al., 2008). Many victims of bullying, especially chronic victims, also do not report their experience and the interventions are, therefore, not implemented (Ladd \& Ladd, 2001; Smith, Shu, \& Madsen, 2001; Unnever \& Cornell, 2004). Numerous studies suggest that victims tend to blame themselves for the bullying, which can lead to feelings of shame and low self-worth, further decreasing the likelihood of reporting (Berger, 2007; Smith, Talamelli, Cowie, Naylor, \& Chauhan, 2004). For example, Smith et al. (2004) interviewed youth who were former or current victims of bullying and inquired about causal attributions regarding their victimization experiences. The majority of victims blamed themselves for what had happened, referring to their own characteristics (e.g., appearance) or behaviour (e.g., annoyed the bully) as reasons for the bullying. Despite victims frequently blaming themselves for the bullying, few bullying intervention programs focus on individual work with victims (e.g., Smokowski \& Kopasz, 2005; Ttofi \& Farrington, 2012).

\subsection{Pathways from Bullying to Long-term Outcome}

Recent research suggests that the enduring psychological distress many people report from bullying victimization early in life has neurobiological underpinnings (Vaillancourt et al., 2013). Bullying victimization in both youth and adult samples has been linked to dysregulations in the stress response system of the body (e.g., in the hypothalamic-pituitary-adrenal axis), leading to lower than normal secretion levels of the stress-hormone cortisol (Monteleone et al., 2009; Vaillancourt et al., 2013). Bullying 
victimization occurring in childhood may also leave lasting biological marks in the physiology of the developing youth, which increase the risk for subsequent health and adjustment problems (McDougall \& Vaillancourt, 2015).

The patterns of neurobiological dysfunctions that have been identified in victims of bullying are the same as those seen in childhood survivors of maltreatment (e.g., sexual and physical abuse; Bremner \& Vermetten, 2001) and adult victims of severe violence (e.g., repeated rape, survivors of the holocaust; Vaillancourt et al. 2013). Furthermore, neuroimaging studies show that social pain (i.e., pain associated with negative social interactions and situations, such as bullying) affects people in a similar biologically manner as does physical pain (i.e., pain stemming from bodily tissue damage and affected by various psychological and social factors). Social pain may, however, be relived more easily and be experienced as more distressing than physical pain which, in turn, can lead to the life-long suffering reported by people who have had extended exposures to social pain (Chen, Williams, Fitness, \& Newton, 2008). Nevertheless, not all victims of bullying experience long-term negative outcomes, which implies there exist important individual differences in the susceptibility to victimization, and that some individuals recover from social pain (Baldry and Farrington, 2005; Bowes, Maughan, Caspi, Moffitt, \& Arseneault, 2010; Hempill, Tollit, \& Herrenkohl, 2014).

Findings from psychological research are in line with those reported from neurobiology. Victims of bullying frequently report experiencing bullying as repeated and prolonged trauma and may develop symptoms of posttraumatic stress from victimization (e.g., Mebane, 2010; Nielsen et al., 2015). Using a theoretical trauma framework for bullying may, therefore, shed light on how victimization leads to 
differences in adjustment outcomes, with important implications for helping victims recover from the detrimental effects of bullying.

1.6.1. Psychological trauma. Trauma has been defined as psychological responses to significantly distressing experiences (American Psychiatric Association, 2013) and has been intrinsic to the study and diagnosis of PTSD. According to the Diagnostic and Statistical Manual of Mental Disorders ( $5^{\text {th }}$ Ed.; DSM-5; American Psychiatric Association, 2013), trauma is defined as responses to direct or indirect exposure to actual or threatened death, serious injury, or sexual violence. Subsequently, people may relive the trauma (e.g., intrusive memories, nightmares), avoid trauma reminders, and experience hyperarousal symptoms (e.g., feeling on edge, difficulties concentrating and sleeping), and negative changes in cognitions and mood. For most individuals, these symptoms decline with time, but for others they persist and are associated with marked distress and impairment across multiple important areas of life (e.g., work, family). PTSD has been described as disrupting the normal recovery from a traumatic experience (American Psychiatric Association, 2013; Resick et al., 2014). Cognitive appraisals of the traumatic experience and various risk and resilience factors, such as social support and coping strategies (e.g., Ozer, Best, Lipsey, \& Weiss, 2008), have been found to influence susceptibility to PTSD and may, thus, also be instrumental in the persistence of psychological distress following bullying victimization and social trauma.

1.6.2. Social trauma. There has been a longstanding debate in the research literature on what events constitute trauma; specifically, what events are significantly stressful to qualify as traumatic (McNally, 2003; van der Kolk, 2007). In quantifying this 
relationship, researchers have examined PTSD-like symptoms in relation to a number of stressful but non life-threatening events. McNally (2003) stated that defining ordinary stressors as traumatic created a "conceptual bracket creep" (p. 231) that undermines the validity of the PTSD diagnoses. Others have argued, however, that the diagnostic criteria for PTSD should be based on symptomatology and distress like other DSM criteria, not on a distinct set of events (Mulder, Fergusson, \& Horwood, 2013). Mol et al. (2005), for example, compared symptoms of PTSD associated with endorsing either a traumatic event (e.g., sexual abuse) or a stressful life event (e.g., divorce, illness of a loved one) as worst event experienced, and found that stressful life events were on average associated with higher levels of PTSD symptoms than traumatic events. PTSD symptoms have also been found following significant negative social experiences (e.g., harsh criticism, rejection, exclusion) especially in the context of social anxiety disorder (SAD; American Psychiatric Association, 2013).

Erwin et al. (2006) compared symptoms of posttraumatic stress resulting from social trauma and life-threatening trauma. Participants with SAD reported hyperarousal and avoidance symptoms to memories of past social trauma whereas participants without SAD did not. Of the individuals with SAD, more than one third met diagnostic criteria for PTSD in relation to the social trauma. Furthermore, many of these individuals rated the social trauma as more distressing than the life-threatening traumatic experiences, even events such as repeated sexual abuse. Similarly, Carleton et al. (2011) examined differences in experiences of social trauma and life-threatening trauma. One in three participants who had experienced both types of events rated the social trauma as more distressing than the life-threatening trauma. Furthermore, posttraumatic stress predicted 
SAD symptoms, suggesting that traumatic responses to social trauma may contribute to the development of SAD. Although social trauma is a broader category of events than bullying and may not involve repetition, symptoms of social anxiety are very common in victims of bullying and bullying victimization has been found to be both a predictor and a consequence of SAD in adolescents (Siegel, La Greca, \& Harrison; 2008).

Research on the role of social trauma in generating social anxiety followed from investigations into recurrent mental self-representations in SAD (Wild, Hackmann, \& Clark, 2007), which Rapee and Heimberg (1997) have suggested to play a role in maintaining SAD symptoms. They argue that when individuals with SAD enter feared social situations they form a mental image of how they believe they appear to others. These images are negatively distorted by the cognitive biases prevalent in SAD (e.g., negative beliefs about themselves, beliefs about high standards for performance in social situations), leading to increased anxiety and distress that elicits avoidance and safety behaviors and further maintains the disorder (e.g., avoid making eye contact with people and thus failing to notice a smile; Clark, 2001). Further investigation into negative selfimages in SAD has revealed that individuals can often link their images to a particular memory of an aversive social experience that occurred closely to the onset of the disorder or to an increase in SAD symptoms, both in terms of themes in the images and sensory details (Hackmann et al., 2000).

The research available on the role of social trauma in SAD onset suggest that it is not the events per se that increase SAD risk but, rather, how people perceive the experience (Hackmann et al., 2000; Levinson, Langer ,\& Rodebaugh, 2013; Rachman, Grüter-Andrew, \& Sharfran, 2000). Individuals with SAD tend to overestimate the cost 
of negative social events, such that having these experiences is indicative of negative self-characteristics (e.g., that there is something wrong with them, that others will always judge them negatively). Furthermore, there is commonly a biased assumption that aversive social events will have negative long-term consequences (e.g., they will never be able to have meaningful interpersonal relationships because of this experience; Stopa \& Clark, 2000; Wilson \& Rapee, 2005). Updating maladaptive interpretations of social trauma is important for treatment effectiveness in cognitive-behavioural therapy (Clark, 2001; Wild \& Clark, 2011). For example, Wilson and Rapee (2005) found that changing maladaptive beliefs about self-characteristics and the probability and cost of negative social experiences led to improvements in SAD symptoms at posttreatment and at 3month follow-up. Similarly, Wild, Hackmann, and Clark (2007) found that imagery rescripting, a technique used to update the meaning of faulty memories, decreased the vividness of negative self-images in patients with $\mathrm{SAD}$ and led to significant symptom improvements. Finally, Nilsson, Lundh, and Viborg (2012) randomized participants with SAD into two groups, one receiving one session of imagery rescripting and the other a reading task. A week later the imagery rescripting group reported more reductions in distress and fears of negative evaluation compared to the reading group, underscoring the effectiveness of focusing treatment on cognitive appraisals.

1.6.3. Cognitive appraisals. Cognitive theories of PTSD maintain that maladaptive appraisals disrupt normal recovery from traumatic experiences (Ehlers \& Clark, 2000). Traumatic experiences are common; but, only about $10 \%$ of the general population develops PTSD following exposure to a traumatic event (McNally, 2003: Resick et al., 2014). Posttraumatic symptoms (e.g., intrusive images) emerge through the 
formation of fear networks in memory that include trauma stimuli (e.g., sound of tires screeching) and responses (e.g., feelings of helplessness) and interpretation of the trauma (e.g., "The accident happened because I could not stop the car"). When the fear networks are activated through trauma reminders, distressing symptoms, such as intrusive thoughts and emotions, emerge. These distressing symptoms elicit avoidance behaviours that aim to reduce the discomfort. With time, however, these avoidance behaviours prevent processing of the traumatic experience in a way that is adaptive for normal recovery (e.g., being able to recognize that fear elicited by a trauma reminder does not represent actual, current threat). Maladaptive cognitive appraisals of the traumatic experience hinder recovery by strengthening the avoidance behaviours and by leading to secondary emotional responses, such as guilt and shame, which further increase the distress associated with the trauma memory (Ehlers \& Clark, 2000; Resick et al., 2014).

Maladaptive trauma appraisals develop from attempts to reconcile the traumatic experience with pre-existing cognitive schemata (Resick et al., 2014). People commonly use one of three techniques: assimilation, accommodation, or over-accommodation. Assimilation refers to adapting the memory and interpretation of the traumatic experience so that it matches prior beliefs, such as blaming oneself for the experience based on the belief that this kind of events only happens to people that have done something wrong. Accommodation represents a more balanced way of reconciliation and refers to accepting that the trauma happened and adapting pre-existing beliefs sufficiently to account for the new trauma-related information. Over-accommodating is radically changing pre-existing beliefs to match the new information, such as thinking that the world is not a safe place anymore because the traumatic experience happened. Importantly, traumatic experiences 
frequently do not fit with pre-existing cognitive schemata, yet they may also confirm previously held negative beliefs. For example, if an individual believes that people are generally dishonest, experiencing an interpersonal trauma may confirm this belief (Ehlers \& Clark, 2000; Resick et al., 2014).

Cognitive appraisals of bullying victims, similar to those seen in PTSD and SAD, are influential for adjustment subsequent to bullying (Ladd \& Ladd, 2001). For example, Troop-Gordon and Ladd (2005) followed 399 children from the ages of nine to11 years, and found that changes in social perception (e.g., how one is perceived by others and how one perceives peers) mediated the association between increase in bullying victimization and increase in depression symptoms. Additionally, a recent longitudinal study (Taylor et al., 2013) examined the development of threat appraisals (i.e., negative self-evaluations and negative evaluations by others) following physical and indirect bullying. Type of threat appraisal was associated with psychological distress two years later; specifically, negative self-evaluations were associated with symptoms of anxiety and depression and being negatively evaluated by others predicted increased aggression.

1.6.4. Risk and resilience factors. A complex interplay between various risk and resilience factors may further influence the trajectories from bullying victimization to long-term adjustment. Resilience is a dynamic construct that refers to abilities and skills to overcome or resist adversity and stress (Fleming \& Ledogar, 2008). Resilient victims of bullying function better than would be expected given the adversities they are faced with; therefore, promoting resilience may mitigate the negative effects of bullying victimization (Bowes et al., 2010). In a systematic review of eight prospective longitudinal studies, Ttofi, Farrington, and Lösel (2014) identified several factors that 
promote resilience against internalizing (e.g., depression) and externalizing (e.g., aggression) problems in victims and bullies later in life. Resilience factors included individual characteristics, such as high academic performance, having strong social and coping skills, and factors related to family and social support, such as having positive family relationships, stability in family structure, and helpful friends.

1.6.4.1. Coping styles. Coping refers to cognitive and behavioural strategies that people use in response to stress. Multiple dimensional frameworks have been proposed to describe coping, but most build upon Lazarus and Folkman (1984)'s Transactional Model of Stress and Coping. According to this model, coping serves two major broad function, including to regulate negative and stressful emotions (i.e., emotion-focused coping) and to alter the stressor (i.e., problem-focused coping). Emotion-focused coping refers to strategies such as avoidance (e.g., substance use) and acceptance, whereas problemfocused coping refers to strategies such as planning and taking steps to resolve the problem. Although Lazarus and Folkman (1984) maintained that coping is a dynamic concept that depends upon the nature of the stressor and individual threat appraisal, others have proposed that people tend to display habitual coping styles that are linked to personality traits and situational outcomes (Litman, 2006).

Garnefski and colleagues (2001) distinguished between nine different coping styles in relation to general stress. These include: (a) self-blame, (b) blaming-others, (c) acceptance (i.e., accepting that the experience occurred), (d) rumination (i.e., repeatedly thinking about the experience, consequences, and associated emotions), (e) catastrophizing (i.e., focusing on the negative aspects of the experience), (f) refocusing on planning and strategizing, (g) putting the stressful experience into perspective, (h) 
positive reappraisal (i.e., positive meaning to the event in terms of personal growth), and (i) positive refocusing (i.e., thinking about joyful and pleasant things instead of the distressing event). Garnefski and Kraaij (2014) examined these coping styles in victims of bullying and found that rumination strengthened the relationship between bullying victimization and symptoms of depression, whereas positive refocusing reduced the impact of bullying victimization on symptoms of anxiety and depression. Similarly, catastrophizing strengthened the relationship between bullying victimization and symptoms of anxiety, whereas positive reappraisal reduced the effect of victimization on anxiety symptoms.

1.6.4.2. Social support. Social support is among the strongest predictors of resilience in the aftermath of trauma (Ozer et al., 2008). Research also suggests that supportive and caring family environment fosters resilience in children who are being bullied (Bowes et al., 2010). Social support from peers is another important resilience factor that buffers against the negative effects of bullying victimization. Being bullied in school during middle childhood and adolescence is predictive of peer dislike and a decrease in popularity (Smith, Talamelli, Cowie, Naylor, \& Chauhan, 2004); but, having one close and supportive friend minimizes the risk of maladjustment (Cook et al., 2010; Hodges, Boivin, Vitaro, \& Bukowski, 1999). Social support from co-workers has, similarly, been found to mitigate the harmful effects of workplace bullying (Cassidy, McLaughlin, \& McDowell, 2014). Social support has also been shown to buffer against the harmful impact of negative childhood experiences on long-term adjustment (Hyman, Gold, \& Cott, 2003). 
1.6.4.3. Continuity. Trauma continuity is associated with greater risk of maladaptive outcomes. Prior trauma history increases the risk of developing PTSD subsequent to a novel traumatic experience, especially for interpersonal trauma (e.g., rape; Ozer et al., 2008), and duration of exposure to traumatic stressors is predictive of PTSD severity (e.g., domestic violence; American Psychiatric Association, 2013; Williams et al., 2007). Chronic bullying victimization is similarly associated with worse outcomes than bullying that is limited to a particular context or time (Ladd \& Ladd, 2001; Smith et al., 2003), as the cumulative effect of repeated or chronic victimization may exhaust the individual's coping resources. Furthermore, failure to control or stop aggressions is likely to have a negative impact on self-appraisals (Ladd \& Ladd 2001).

1.6.4.4. Event centrality. The salience of maladaptive appraisals of a traumatic experience may rest on the extent to which the individual perceives that the traumatic experience has become central to their personal identity and understanding of the world and may, therefore, also be influential on distress following bully victimization (Groleau, Calhoun, Cann, \& Tedeschi, 2013). Traumatic experiences that become too prominent and central, and a reference for one's autobiographical memory, increase the risk of developing PTSD (Berntsen \& Rubin, 2006; McNally, 2003). Similar to negative selfimages in SAD, highly rehearsed and accessible self-defining trauma memories are associated with distress, leading to avoidance, and maintaining and strengthening maladaptive appraisals and PTSD symptoms (Bernsten \& Rubin, 2006). Conversely, perception of trauma centrality can also lead to posttraumatic growth (PTG) and positive appraisals, which are associated with greater well-being (Boals \& Schuttler, 2011; 
Roland, Currier, Rojas-Flores, Herrera, 2014). This inverse relationship may be explained by individual differences in resilience and coping styles (Schuettler \& Boals, 2011).

1.6.4.5 Anxiety sensitivity. Anxiety sensitivity (AS) refers to a cognitive vulnerability characterized by somatic, cognitive, and social fears of anxiety sensations based on the premise that they have negative consequences (i.e., "fear of fear"; Reiss \& McNally, 1985). AS is a risk and maintenance factor for various anxiety-related disorders, particularly panic disorder and PTSD (Asmundson \& Stapelon, 2008; Deacon \& Ambramowitz, 2006; Taylor et al., 2001). Marshall, Miles, and Stewart (2010) found a reciprocal relationship between levels of AS and PTSD symptoms; specifically, higher levels of AS predicted PTSD symptom severity and greater PTSD symptom severity predicted an increase in AS over time. Furthermore, evidence suggests that traumafocused treatment leads to reductions in AS, which, in turn, is associated with reductions in PTSD symptoms (Gutner, Nillni, Suvak, Wiltsey-Stirman, \& Resick, 2013).

1.6.4.6 Distress tolerance. Distress tolerance is the perceived or actual capacity to withstand aversive emotional states (Brown et al., 2009). Individuals with low distress tolerance may respond to stressful experiences in maladaptive ways (e.g., avoidance) and be more prone to develop PTSD and various other psychological concerns, such as substance use disorder (Zvolensky, Vujanovic, Bernstein, \& Leyro, 2010). Lower distress tolerance has also been linked to greater severity of PTSD symptoms (Marshall-Berenz, Vujanovic, Bonn-Miller, Bernstein, \& Zvolensky, 2010) and may be a mediator in the relationship between AS and PTSD (Berenz, Vujanovic, Coffey, \& Zvolensky, 2012).

1.6.4.7. Intolerance of uncertainty. Distress tolerance encompasses another related cognitive construct, intolerance of uncertainty (IU). IU is the tendency to fear 
ambiguous or uncertain situations. High levels of IU are a risk and maintenance factor for various emotional problems, including PTSD (Barlow, 2000). Oglesby, Boffa, Short, Raines, and Schmidt (2016) found that pre-trauma levels of IU predicted the development of posttraumatic stress following a traumatic experience, even after controlling for pretrauma levels of AS. Furthermore, reductions in IU are associated with improvement in anxiety and depression symptoms (Boswell, Thompson-Hollands, Farchione, \& Barlow, 2013).

\subsection{Cognitive Processing Therapy}

CPT focuses on identifying and updating dysfunctional trauma interpretations, based on the premise that maladaptive appraisals represent "stuck-points" between prior beliefs and post-trauma beliefs (i.e., assimilation or over-accommodation) that affect mood and behaviour in negative ways (Resick et al., 2014). The goals of treatment are to: (a) address secondary emotions construed by maladaptive appraisals, such as shame, guilt, and sadness, (b) address the content of the trauma appraisals, and (c) examine level of accommodation of the trauma memory in relation to prior cognitive schema. These goals are achieved through psychoeducation, exposure exercises through written accounts of the traumatic experience, and cognitive restructuring (e.g., Socratic questioning; Shipherd, Street, \& Resick, 2006).

Manualized CPT is typically conducted over 12 60-90 minute sessions once or twice per week; however, adjustments can be made to how the treatment is conducted in terms of session length and time spent on treatment modules (Galovski, Blain, Mott, Elwood, \& Houle, 2012; Resick et al., 2014). One study found that CPT delivered through teleconferencing was equally effective to in-person CPT in reducing symptoms 
of PTSD (Morland et al., 2015; Olthuis et al., 2016). Research confirms that CPT is superior to inactive control conditions, such as waitlists, in reducing PTSD symptoms and comorbid depression, and equally effective to prolonged exposure therapy for PTSD (Asmundson et al., 2019; Zappert \& Westrup, 2008). Compared to prolonged exposure therapy, CPT may, however, be superior in reducing trauma-related guilt (Nishith, Nixon \& Resick, 2005; Resick et al., 2002). Research further provides support for the emphasis in CPT on targeting maladaptive trauma appraisals. Resick et al. (2008) dismantled CPT into components comprised of cognitive restructuring of maladaptive appraisals and written exposures to the traumatic memory. They compared three groups of female victims of sexual and physical assault that received 12 sessions of either cognitive restructuring (CPT-C) only, written exposures (WA) only, or manualized CPT that included both components. Participants in all groups improved from baseline to posttreatment in symptoms of PTSD and depression; but, symptom changes were significantly greater in the CPT-C and CPT groups compared to the WA group. The authors concluded from these findings that cognitive restructuring of maladaptive trauma appraisals represented the active mechanisms of change in CPT. A subsequent study by Gallagher and Resick (2012) supported this conclusion, finding that cognitive changes in hopelessness moderated PTSD symptom improvement in female victims of sexual assault but not habituation to distress associated with the trauma memory. Finally, a qualitative study by Price, MacDonald, Adair, Koerner, and Monson (2014) with male and female military service members found that trauma appraisals shifted from negative to more positive following the course of treatment, supporting the proposed cognitive mechanisms of CPT. 
CPT has been implemented successfully to treat PTSD (i.e., participants no longer meet diagnostic criteria for PTSD) in diverse samples, including victims of rape, military service members, individuals with comorbid conditions, including depression, substance use problems, and personality disorders, and adults with complex and persistent distress associated with childhood trauma (Chard, Ricksecker, Healy, Karlin, \& Resick, 2012; Chard, 2005; Forbes et al., 2012; Resick et al., 2014; Walter, Bolte, Owens, \& Chard, 2012). Effect sizes, such as Hedges g, are typically large, and vary from one to 1.3 between studies. Similar to other active treatments for PTSD, such as prolonged exposure therapy, treatment dropout rates in CPT (i.e., completing less than nine of 12 , or $75 \%$ of treatment sessions) are typically high and vary from approximately 15-30\% (Forbes et al., 2012; Imel, Laska, Jakupcak, \& Simpson, 2015; Rizvi, Vogt, \& Resick, 2009). Few studies have examined factors associated with treatment response and dropout in CPT. Rizvi, Vogt, and Resick (2009) examined cognitive and affective predictors of treatment efficacy and dropout in a sample of female victims of sexual assault diagnosed with PTSD that received group CPT. Approximately $22 \%$ of the women dropped out of treatment after completion of a median of four of 12 sessions. Higher treatment dropout was associated with lower intelligence, education, and age. Higher levels of guilt and depression at baseline were, however, associated with more improvement in PTSD symptoms at the end of treatment for treatment completers (i.e., completed more than $75 \%$ of the treatment). Similarly, Galovski et al. (2016) examined patterns and predictors of symptom change in male and female victims of interpersonal violence with PTSD and comorbid symptoms of depression that received four to 18 sessions of individual CPT, dependent upon recovery (i.e., whether met treatment goals and no longer met diagnostic 
criteria for PTSD). Recovery fell into one of two patterns for most participantsconsistent responders reported steady, linear decrease in symptoms throughout treatment, and initial responders had lower symptom levels at the beginning of treatment and reported decelerating symptom change as treatment progressed. Symptoms of PTSD and depression declined simultaneously for most participants; but, higher levels of both symptoms at baseline were associated with slower recovery. A small subset of participants (i.e., approximately $7.2 \%$ of the sample) reported partial recovery from PTSD and depression. Participants in this group were on average older than other participants, had the most severe levels of PTSD and depression symptoms at baseline, and received more CPT sessions than other participants but still experienced less overall change. Approximately 27\% (19 of 69) of participants dropped out of treatment. All of the dropouts came from the group of participants that reported consistent decrease in PTSD and depression symptoms. Logistic issues (e.g., childcare, employment), rather than treatment specific issues, were stated as reasons for leaving treatment early.

Taken together, evidence from the aforementioned studies demonstrates that CPT is an effective treatment that can successfully be applied in both manualized and modified versions to treat PTSD and comorbid conditions, such as depression. Similar to other active treatments for PTSD, dropout rates in CPT are rather high, which underscores the importance of carefully monitoring client progress throughout treatment to optimize treatment effectiveness. Among variables associated with higher dropout in CPT are younger age and logistic issues. Delivering CPT through the internet may solve some of these issues. Internet-delivered treatment may be more appealing for younger 
people and the increased flexibility of completing treatment modules at home through the internet may reduce treatment attrition.

\subsection{Internet-Delivered Treatment for PTSD}

Internet-delivered cognitive behavioural therapy (iCBT) protocols for PTSD typically include cognitive restructuring, de-arousal strategies (e.g., breathing retraining), and in-vivo, imaginal, or narrative exposure exercises. Although clients complete treatment modules on their own, iCBT often includes regular contact with a mental health care provider through email, telephone, or other media that offers client support and guidance (Ivarsson et al., 2014). Internet-delivered treatments for PTSD are increasingly promoted as a cost-effective way to improve access to psychological treatment (Andersson, Rozental, Shafran, \& Carlbring, 2018; Sibjbranij et al., 2016). Delivering treatment through the internet provides more accessibility to individuals living in rural areas and individuals with restricted mobility, and more acceptability for those who may not seek mental health services because of fear of stigmatization (Sijbrandij et al., 2016). Internet-delivered treatments are also more flexible than in-person treatments, as session content can be accessed and completed outside of regular office hours (Sijbrandij et al., 2016).

Several randomized controlled trials have examined the effectiveness of iCBT for PTSD. A recent meta-analysis (Sijbrandij et al., 2016) summarized the results of 12 randomized controlled trials that compared iCBT that consisted of psychoeducation, cognitive restructuring, and some sort of exposures (e.g., written exposures, audiotapes to record imaginal exposures), to active and inactive control conditions, including waitlist group, treatment as usual, and online supportive counselling. iCBT was found to be 
superior to waitlist and treatment as usual in reducing PTSD symptoms and comorbid symptoms of depression $(g=0.71)$, and was somewhat more effective than supportive counselling and psychoeducation $(g=0.28)$. The effects of iCBT were greater when participants completed eight or more iCBT sessions and received treatment that was therapist-guided rather than self-guided. The relative low number of studies included in the meta-analysis, significant heterogeneity in the design and type of treatment in the studies included, and that no comparisons were made with in-person CBT undermines the findings of this meta-analysis. However, The Canadian Agency for Drugs and Technologies in Health (CADTH) conducted a peer-reviewed summary of the research evidence on the effectiveness of iCBT for PTSD (Young \& Campbell, 2018). The summary, which included three systematic reviews with meta-analyses and two additional randomized controlled trials, concluded that iCBT was more effective for reducing symptoms of PTSD than inactive control conditions, and internet-delivered supportive counselling, and produced comparable effects to CBT for PTSD delivered inperson.

\subsection{Behavioural Stress Management}

Behavioural stress management refers to various relaxation techniques that aim at reducing psychological distress. Stressors are threats to psychological, biological, and physiological stability (or homeostasis) which have various negative effects on health and wellbeing, including increased risk of depression and various physical health problems. Several empirically-supported behavioural stress management techniques exist that aim at reducing the physical arousal associated with stress (Taylor, 2017). Progressive muscle relaxation (PMR) reduces stress and anxiety through repeated alterations of muscle 
tensing and relaxing. The individual intentionally tenses his or her muscles for few seconds while focusing on the different experiences of tension and relaxation. Diaphragmatic breathing is another stress management technique that involves manipulation of breath. Diaphragmatic breathing is characterized by expansion of the abdomen through slower and deeper inhalation and exhalation. Guided imagery (GI) is yet another effective stress management technique that induces relaxation through symbolic, calming images, such as of a landscape. Attention is brought to the chosen mental image by engaging in imagery exploration of the sensory elements (e.g., auditory, visual, olfactory) involved with the image (Varvogli \& Darviri, 2011). Daily practices of behavioural stress management techniques have been found effective for various psychological and physical issues, including pain management, generalized anxiety disorder, and repetitive and intrusive thoughts (Baird, Carol, Sands \& Laura, 2004; Feldman, Greeson, \& Senville, 2010; Pawlol \& Jones, 2002). Internet-delivered behavioural stress management treatment has also been found to be effective for reducing symptoms of severe health anxiety and panic disorder (Carlbring, Ekselius, \& Andersson, 2003; Hedman et al., 2014).

Relaxation techniques are frequently incorporated into treatment protocols for PTSD, such as breathing retraining in prolonged exposure therapy; but, relaxation techniques are also effective in reducing posttraumatic distress in and of themselves (Taylor et al., 2003). Marks, Lovell, Noshirvani, Livanou and Trasher (1998) found that 10 weeks of relaxation training, which included daily relaxation exercises using taped recordings, reduced global severity of PTSD symptoms as measures by the ClinicianAdministered PTSD Scale (CAPS-2). Relaxation training produced lower effects on 
PTSD symptoms, however, than exposure therapy, cognitive therapy, and combined cognitive and exposure therapy. Similarly, Taylor et al. (2003) compared relaxation training consisting of 60-90 minute scripted relaxation exercises for eight weeks to exposure treatment and eye movement desensitization and reprocessing (EMDR). They found that relaxation training produced clinically significant reductions in PTSD symptoms comparable to EMDR from pre-treatment to three month follow-up $\left(\eta^{2}=.47\right)$; however, exposure therapy produced greater levels of clinically significant reductions at post-treatment and three month follow-up than relaxation training and EMDR $\left(\eta^{2}=.59\right)$. All treatments had similar dropout rates and were rated equally credible by participants. The authors suggested that relaxation training might be effective in reducing hyperarousal symptoms, which, in turn, facilitates approaching avoided situations (i.e., in-vivo exposures) that lead to reductions in PTSD symptoms and distress.

\subsection{Summary}

Victims frequently suffer pervasive and long-term negative consequences from bullying experiences. While focusing psychological treatment for victims of bullying on specific symptoms of mental health difficulties (e.g., depression) may be helpful, the onset of problems can often be linked directly to the bullying. Thus, conceptualizing bullying victimization as trauma and the negative psychological impact of victimization as a form of posttraumatic stress could be a viable option for advancing treatment. Using a CPT framework, bullying victimization leads to formation of fear networks in memory that become highly accessible with time and promote prolonged distress. The fear networks get activated through various reminders of the bullying victimization experience which leads to avoidance behaviours, such as social withdrawal and 
distraction from distressing thoughts and emotions. Maladaptive interpretations of the meaning and implications of the bullying (e.g., "I can't trust people") may disrupt recovery from victimization through generation of secondary emotions, such as shame and hopelessness, that increase avoidance behaviours and hinder reappraisals and processing of the victimization experience. Various risk and resilience factors, such as trauma centrality, coping styles, and social support may further influence recuperation from bullying victimization (Takisawa et al., 2014 ).

CPT is an effective treatment for PTSD and comorbid depression that has been applied successfully in both the manualized and modified versions (Asmundson et al., 2019). Adapting CPT to an internet-delivered format could, furthermore, be beneficial as internet-delivered treatments improve accessibility to therapy (e.g., to people in rural areas, can be provided at a lower cost) and acceptability for those that may avoid seeking mental health services due to fear of stigmatization (Sijbrandij et al., 2016). To establish that CPT is effective for updating maladaptive appraisals from bullying victimization experiences and associated symptoms of psychological distress, rigorous testing comparing the efficacy of the treatment to no treatment and an active control treatment is needed. Stress management techniques, including relaxation, are one option for an active control comparison that have been found helpful to reduce the global severity of PTSD symptoms, although to a lesser extent than exposure therapy, (Taylor, 2017; Taylor et al., 2003).

Research underscores that victims of bullying tend to blame themselves, attributing their own personal characteristics and failure to stop the bullying for their negative experiences, (Smith et al., 2004), which leads to shame and decreases the 
likelihood of reporting (Berger, 2007). Problems with implementation of bullying interventions frequently arise at schools and workplaces (Ttofi \& Farrington, 2011), and few intervention programs focus on individual work with the victims. Effective, victimtailored and accessible interventions are, therefore, needed.

\section{CURRENT INVESTIGATION AND METHODS}

\subsection{Purpose}

This investigation was a randomized controlled trial involving a therapist-guided internet-delivered CPT for maladaptive appraisals associated with social trauma and bullying victimization compared against a therapist-guided stress management (SM) and a waitlist group in an adult sample of participants that self-identified as victims of bullying. The CPT followed the manual of Resick et al. (2014) in terms of the organization of session content; but, it was modified to fit an internet-delivered format and the context of social trauma and bullying victimization. The purpose of the study was to test whether CPT could be used to effectively update maladaptive appraisals and psychological distress resulting from bullying victimization, and to examine various individual and environmental risk and resilience factors that might influence the relationship between bullying victimization and long-term outcomes.

\subsection{Hypotheses}

1. Participants in the CPT group will report greater reductions in self-reported maladaptive appraisals from exposure to bullying victimization over time compared to participants in the SM group and the waitlist. 
2. Participants in the CPT group will report greater reductions in psychological distress (i.e., symptoms of posttraumatic stress, social anxiety, depression, general stress, and anxiety, anger, distress tolerance, anxiety sensitivity, and intolerance of uncertainty) over time compared to participants in the SM group and the waitlist.

3. Level of resilience, perceived social support, and coping styles will mediate the relationship between perceived event centrality and strength of maladaptive appraisals from bullying victimization at baseline.

4. Strength of maladaptive appraisals at baseline will predict psychological distress, and this relationship will be moderated by number of bullying victimization experiences.

\subsection{Participants}

Ethical approval was obtained from the University of Regina Research Ethics Board (REB\#2017-063, see Appendices A-C). Participants were recruited from Saskatchewan through internet social media platforms (e.g., Facebook), local media, and advertisement posters. Eligible participants were residents of Saskatchewan between the ages of 18-65 years who reported being exposed to bullying (i.e., either as victims only or bully-victims) and endorsed distress associated with this experience, had access to a computer and Internet, and provided an emergency contact (e.g., a family physician). They could not be currently engaged in psychotherapy or talk therapy that focused specifically on their bullying experiences. If on a waitlist for psychotherapy or talk therapy, participants were asked to refrain from entering therapy for the duration of the study. Participants could not be hospitalized or taking antipsychotic medication, and had 
to be at low risk for suicide. If participants were engaged in pharmacological treatment for psychological concerns, they were asked to remain on the same dosage level for the duration of the trial. The procedure for assessing eligibility is described below.

Eligible participants were randomized into one of the two experimental groups, which received either internet-delivered CPT or internet-delivered SM, or to the waitlist group. Participants in CPT and SM could choose to complete the treatment modules in six to 12 weeks. The waitlist group received either CPT or SM after six weeks, and this data was included in the analyses. Number of participants for the trial was based on the sample sizes of previously published studies of CPT (Resick et al., 2002; Resick et al., 2008), and recommendation about sufficient sample sizes when using hierarchical linear modeling (HLM; which was the statistical method selected to analyse the data and is described below; Maas \& Hox, 2005), because the statistical power for HLM cannot be easily predicted as it depends on various factors and assumptions at each level of the analysis (Kreft \& DeLeeuw, 1998). Predicting an attrition rate of approximately $20 \%$ (i.e., Resick et al., 2002), with 30 treatment completers per group, the recruitment target for the trial was approximately 100 individuals.

\subsection{Acceptability testing}

Acceptability testing on the CPT and SM protocols was conducted to inform participant recruitment, make adjustments to the treatment modules, and inquire about participant experiences completing the protocols. Prior to participant recruitment, three graduate student volunteers completed the 12 internet-delivered therapy modules in each treatment group and provided feedback on language and acceptability and adjustments were made accordingly. All eligible participants, whether they completed their assigned 
treatment protocol or not, were emailed a short survey about the content of their assigned treatment (see Appendices U and V). Participants that dropped out of the trial before completing at least one session of CPT or SM were queried about their reasons for discontinuing participation. Participants who completed the majority of the CPT or SM sessions were asked about treatment satisfaction, module content, and therapist support.

\subsection{Measures}

\subsubsection{Screening measures.}

2.5.1.1. Retrospective Bullying Questionnaire (RBQ; Schäfer et al., 2004; see Appendix D). The RBQ is a comprehensive assessment tool with 46 multiple-choice and open-ended questions that evaluate history and impact of bullying experiences using selfreport. The RBQ has four sections. Sections one and two consist of questions of recalled bullying experiences in primary and secondary school (i.e., ages 4-11 years and ages 1118 years). Section three includes questions about general reactions and distress associated with bullying experiences (e.g., "Which were the main ways you used to cope with the bullying?"; "Do you ever have sudden vivid recollections or "flashbacks" to the bullying event(s)?"). Section four consists of questions about bullying experiences in the past six months.

Under each section, three types of bullying behaviours (i.e., physical aggressions, verbal aggressions, and indirect bullying/social manipulations) are evaluated in terms of frequency, duration, and seriousness of the recalled experience. In line with Blood and Blood (2016), and with permission from the authors, the present trial also evaluated experiences of cyber-bullying using the same questions about frequency and duration. Furthermore, a question about perceived social support from friends, parents, or 
teacher/school environment during the bullying experiences was also added to section three (i.e., question 36).

Based on item endorsement, participants are classified into victims if they: (a) report being bullied in one or more ways (i.e., physical, verbal, indirect, or cyber), (b) endorse that bullying victimization occurred "sometimes", "frequently", or "constantly", and (c) indicate that the intensity of the victimization was "quite serious" or "extremely serious". Responders are classified as bullies if they endorse that they: (a) bullied others “sometimes", "frequently," or "constantly", and (b) were never victims of bullying. Bully-victims are classified by responses endorsing: (a) being bullied "sometimes", "frequently", or "constantly", (b) perceiving the victimization to be "quite serious" or “extremely serious", and (c) also bullying others. Classifications of bystanders and uninvolved (i.e., responses indicating no direct involvement in bullying at all) can also be obtained from the RBQ. The test-retest reliability of the RBQ over a two-month period has been reported as good, $r=.88$ for section 1 and $r=.87$ for section 2 (Shäfer et al., 2004).

2.5.1.2. Life Events Checklist for DSM-5 (LEC-5; Weathers et al., 2013; see Appendix E). The LEC-5 assesses trauma history with 17 items that list various traumatic events (e.g., natural disaster, serious accident). For the purpose of this trial, bullying victimization was added as the $18^{\text {th }}$ item on the list of traumatic events. Responders to LEC-5 items are asked to indicate whether they experienced each event directly (i.e., happened to them or they witnessed it happen to someone else) or indirectly (i.e., learned about it happening to a close family member or friend). From this list of events, participants are asked to identify the worst event and to rate subsequent questionnaire 
items based on this experience. To be eligible to participate in the trial, participants had to endorse that they experienced bullying victimization and that this experience had a continued and significant negative impact on their lives. They were asked to respond to symptom questionnaires based on their bullying victimization experience.

2.5.1.3. P4 screener (Dube, Kroenke, Bair, Theobald, \& Williams, 2010; see Appendix F). The P4 screener is a short assessment tool for suicide risk that is administered to individuals who endorse thoughts of hurting themselves. It has four items that ask about past suicide attempts, plans for suicide, probability of completing suicide, and preventive factors. Based on individual's responses to the items, suicide risk is classified as minimal, lower, and higher. Individuals whose responses indicated higher risk were ineligible to participate in this trial and received a phone call from the primary investigator to inquiry about risk to self and current available supports, and to provide them with contact information for community services and crisis resources. Suicide risk of eligible participants was monitored throughout the trial with weekly depression symptom measure and internet therapist contact.

\subsubsection{Primary outcomes.}

2.5.2.1. Posttraumatic Stress Disorder Checklist for DSM-5 (PCL-5; Weathers et al., 2013; see Appendix G). The PCL-5 measures PTSD symptoms in the past month with 20 items rated on a five point Likert scale ranging from 0 (not at all) to 4 (extremely).

Total scores are derived from the sum of item scores and vary from 0 to 80 , with clinically significant symptoms of PTSD identified by total scores of approximately 30 or higher. For a provisional DSM-5 diagnosis of PTSD, individuals must endorse items from each category of symptoms; namely, at least one re-experiencing symptom, one 
avoidance symptom, two hyperarousal symptoms, and two negative changes in cognition and mood. For the purpose of the present trial, participants were asked to evaluate their PTSD symptoms based on their worst bullying victimization experience. The PCL-5 has sound psychometric properties, including high internal consistency $(\alpha=.94)$ and good test-retest reliability ( $r=.82$; Blevins, Weathers, Davis, Witte, \& Domino, 2015). In the present sample, the internal consistency of the PCL-5 was excellent $(\alpha=.96)$,

2.5.2.2. Posttraumatic Cognitions Inventory (PTCI; Foa et al., 1999; see Appendix H). The PTCI is comprised of 33 items that measure appraisals of traumatic experiences. Items are rated on a seven point Likert-scale ranging from 1 (totally disagree) to 7 (totally agree). Items are summed to obtain a total score (0-231). The PTCI has three subscales that assess appraisals related to self-blame with five items (e.g., "The event happened because of the way I acted”), negative self-beliefs with 21 items (e.g., The event happened to me because of the sort of person I am"), and negative beliefs about the world with seven items (e.g., "People can't be trusted"). Foa et al. (1999) found that the median total score for non-traumatized individuals was 45.5 , for traumatized individuals without PTSD was 49.0, and for traumatized individuals with PTSD it was 133.0. Participants in this study were asked to evaluate the appraisals from their worst bullying victimization experience using PTCI items. The PTCI has sound psychometric properties, including high test-retest reliability ( $p$ s from .74 - .89) and internal consistency ( $\alpha s$ for subscales and total scale from .86 - .97; Foa et al., 1999). Internal consistency in the present sample ranged from good to excellent for the total scale $(\alpha$ $=.96)$ and the three subscales $(\alpha s=.76-.95)$. 
2.5.2.3. Social Interaction Phobia Scale (SIPS; Carleton et al., 2009; see Appendix I). The SIPS is a self-report measure of social anxiety symptoms (e.g., fear of negative evaluation in social interaction and performance situations). The SIPS has 14 items that are rated on a five point Likert scale that ranges from 0 (not at all) to 4 (extremely). The items are summed to yield a total, and scores on three subscales, including Social Interaction Anxiety (e.g., "I find myself worrying that I won't know what to say in social situations"), Fear of Overt Evaluations (e.g., "I feel self-conscious if I have to enter a room where others are already seated"), and Fear of Attracting Attention (e.g., "I am worried people will think my behaviour odd"). The clinical cut-off score for significant symptoms is 21 or higher. The SIPS has good psychometric properties, including high internal reliability ( $\alpha s=.89-.91$ for the total scale and subscales in a clinical sample, and $\alpha s=.77-.87$ in a non-clinical sample) and test-retest reliability ( $r s$ >.79; Menatti et al., 2015). Internal consistency in the present sample ranged from good to excellent for the total and subscales $(\alpha s=.80-.97)$.

2.5.2.4. Depression Anxiety Stress Scales-21 (DASS-21; Lovibond \& Lovibond, 1995; see Appendix J). The DASS-21 measures symptoms indicative of depression, anxiety, and stress using 21 items that are rated on a four point Likert scale ranging from 0 (did not apply to me at all) to 3 (applied to me very much or most of the time). Items are summed to obtain a total (i.e., general distress) and relevant subscale (i.e., depression, anxiety, and stress) scores. For severity scores, which are based on the longer version of the scale (i.e., DASS; Lovibond \& Lovibond, 1995), the subscale total scores are multiplied by two. For depression, scores from 14-20 indicate moderate symptom severity, 21-27 severe symptoms, and 28 and higher extremely severe symptoms. For 
anxiety, scores from 10-14 suggest moderate anxiety, 15-19 severe anxiety, and 20 and higher extremely severe anxiety. On the stress subscale, scores between 19 and 25 indicate moderate stress, 26-33 severe stress, and 34 and higher extremely severe stress. The DASS-21 has good internal consistency $(\alpha s=.82-.93)$ and convergent validity (e.g., $r=.78$; Henry \& Crawford, 2005). Internal consistency in the current sample ranged from good to excellent $(\alpha s=.84-.95)$ for the total scale and three subscales.

2.5.2.5. Dimensions of Anger Reactions-5 Scale (DAR-5; Forbes et al., 2014a, 2014b see Appendix K). The DAR-5 measures affective and behavioural expressions of anger and associated interference of anger with functioning. The scale has five items rated on a five point Likert scale ranging from 1 (none of the time) to 5 (all of the time). A total score is obtained by summing item scores. Total scores range from five to 25 . Scores of 12 and higher are indicative of more frequent, intense, and long lasting anger and aggression responses that interfere with functioning and might need clinical attention. The DAR-5 has sounds psychometric properties, including high internal consistency ( $\alpha s$ $=.86-.90)$ and high concurrent validity ( $r s=.41-.72$; Forbes et al., 2014a, 2014b). The internal consistency in the current sample was good $(\alpha=.87)$.

2.5.2.6. Distress Tolerance Scale (DTS; Simons \& Gaher, 2005; see Appendix L). The DTS measures tolerance of emotional distress with 15 items rated on a five- point Likert scale ranging from 1 (strongly agree) to 5 (strongly disagree). The DTS has four subscales, including Tolerance (e.g., "Feeling distressed or upset is unbearable to me"), Absorption (e.g., "My feelings of distress are so intense that they completely take over"), Appraisal (e.g., "My feelings of distress or being upset are not acceptable”), and Regulation (e.g., "I'll do anything to avoid feeling distressed or upset"). Items are 
summed and averaged to arrive at a total score and subscale scores, with higher scores indicating a higher tolerance for distress. The DTS has good psychometric properties, including good internal consistency $(\alpha=.89)$ and adequate discriminant and convergent validity (Simons \& Gaher, 2005). Internal consistency in the current sample ranged from acceptable to good $(\alpha s=.71-.89)$ for the total scale and four subscales.

2.5.2.7. Anxiety Sensitivity Index-3 (ASI-3; Taylor et al., 2007; see Appendix M). The ASI-3 is a measure of AS comprised of 18 items that are rated on a five point Likert scale ranging from 0 (agree very little) to 4 (agree very much). The ASI-3 has three subscales, including Cognitive Concerns (items 2, 5, 10, 14, 16, 18; e.g., "It scares me when I am unable to keep my mind on a task"), Social Concerns (items 1, 6, 9, 11, 13, 17; e.g., "It is important to me not to appear nervous"), and Physical Concerns (items 3, 4, 7, 8, 12, 15; e.g., "When I feel pain in my chest, I worry that I'm going to have a heart attack"). Items are summed to arrive at a total and subscale scores. Total scores of 23 and above indicate high AS and 17 and above moderate levels of AS (Allan et al., 2014). The ASI-3 has sound psychometric properties, including good internal consistency $(\alpha s>.70)$ and convergent validity (e.g., $r$ s ranging from .59 - .92 in a clinical sample; Taylor et al., 2007). Internal consistency in the current sample ranged from good to excellent ( $\alpha s=.86$ - .92) for the total scale and three subscales.

2.5.2.8. Intolerance of Uncertainty Scale, Short Form (IUS-12; Carleton, Norton, \& Asmundson, 2007; see Appendix N). The IUS-12 measures IU with 12 items that are rated on a five point Likert scale ranging from 1 (not at all characteristic of me) to 5 (entirely characteristic of me). Items are summed to arrive at a total score, with higher scores indicating higher levels of IU. The IUS-12 has two subscales that measure 
prospective (items 1, 2, 4, 5, 8, 9, 11; e.g., "I always want to know what the future has in store for me") and inhibitory (items 3, 6, 7, 10, 12; e.g., "The smallest doubt can stop me from acting") IU. The measure has good psychometric properties, including high internal consistency $(\alpha s=.85-.91)$ and discriminant validity (Carleton et al., 2007; McEvoy \& Mahoney, 2011). The internal consistency in the current sample was excellent for the total scale and subscales $(\alpha s=.94-.96)$.

\subsubsection{Secondary measures}

2.5.3.1. Centrality of Event Scale-short (CES-short; Berntsen \& Rubin, 2006. See Appendix O). The CES-short measures event centrality (i.e., the extent to which an event has become central to an individual's identity and life story) with seven items (e.g., "I feel that this event has become part of my identity") that are rated on a five point Likertscale ranging from 1 (totally disagree) to 5 (totally agree). Items are summed to obtain a total score, with higher scores indicating higher levels of perceived event centrality. The CES-short has sound psychometric properties, including high internal consistency ( $\alpha$ $=.92$; Bernsten \& Rubin, 2006; Boals \& Schuettler, 2011). Internal consistency in the current sample was excellent $(\alpha=.93)$.

\subsubsection{Cognitive Emotion Regulation Questionnaire-short (CERQ-short;}

Garnefski \& Kraaij, 2006; See appendix P). The CERQ-short measures cognition and emotion regulation strategies with 18 items that are rated on a five point Likert scale ranging from 1 (almost never) to 5 (almost always). The CERQ-short has nine subscales, each with two items: (a) self-blame (e.g., "I feel that I am the one who is responsible for what has happened"), (b) acceptance (e.g., "I think that I have to accept that this has happened"), (c) rumination (e.g., "I am preoccupied with what I think and feel about what 
I have experienced"), (d) positive refocusing (e.g., "I think of pleasant things that have nothing to do with it"), (e) planning (e.g., "I think about how to change the situation"), (f) positive reappraisal (e.g., "I think I can learn something from the situation"), (g) putting into perspective (e.g., "I tell myself there are worse things in life"), (h) catastrophizing (e.g., "I keep thinking about how terrible it is what I have experienced"), and (i) otherblame (e.g., "I feel that basically the cause lies with others"). Items on the subscales are summed to arrive at a total subscale score, with higher scores indicating stronger tendency to engage in the cognitive or emotion regulation strategy. The CERQ-short has adequate to good internal reliability $(\alpha s=.67-.81)$ and convergent validity (Garnefski \& Kraaij, 2006). Internal consistency in the current sample ranged from good to excellent $(\alpha s=.71-.94)$.

2.5.3.3. Brief Resilience Scale (BRS; Smith et al., 2008; see Appendix Q). The BRS measures the ability to bounce back and recover from stress. The BRS has six items that are rated on a five point Likert scale ranging from 1 (strongly disagree) to 5 (strongly agree). Three of the six items on the BRS are positively worded (e.g., "I tend to bounce back quickly after hard times") and three are negatively worded (e.g., "It is hard for me to snap back when something bad happens"). The negatively worded items are reverse coded in the scoring. Items are summed up and divided by six to arrive at a total score, ranging from 1 to 5. Scores ranging from 1.0 - 2.9 are indicative of low resilience, $3.0-$ 4.3 of medium resilience, and $4.31-5$ of high resilience. The BRS has good psychometric properties, including high reliability ( $\alpha$ s from .80-.91) and good convergent and discriminant validity (Smith et al., 2008). The internal consistency in the current sample was excellent $(\alpha=.94)$. 


\subsubsection{Multidimensional Scale of Perceived Social Support (MSPSS; Zimet,}

Dahlem, Zimet, \& Farley, 1988; see Appendix R). The MSPSS was used to assess how participants perceive their current social support. The MSPSS has 12 items rated on a seven point Likert scale ranging from 1 (very strongly disagree) to 7 (very strongly agree). Items fall into one of three subscales that pertain to source of perceived social support, including Family (four items), Friends (four items), and Significant Other (four items). Items are summed and divided by four to arrive at subscale scores, with higher scores indicating more perceived support. Mean total scores ranging from 1 to 2.9 are considered low perceived social support, 3-5 moderated support, and 5.1-7 high support. Items on the MSPSS can also be summed across subscales and divided by 12 for a total score that represents global satisfaction with social support. The MSPSS has sound psychometric properties, including high internal reliability ( $\alpha$ s from .84-.92) and good construct validity in both clinical and non-clinical samples (Zimet et al., 1988; Clara, Cox, Enns, Murray, \& Torgrudc, 2003). The internal consistency in the current sample was excellent for the total scale and three subscales $(\alpha s=.95-.96)$.

\subsection{Procedures}

The procedures of the trial involved five steps: (a) eligibility screening, (b) completion of baseline measures, (c) randomization, (d) participation in the experimental phase, and (e) follow-up. Description of each step is provided below and an overview of the procedures is presented in Figure 1.

2.6.1. Eligibility screening. Individuals interested in participating in the trial were directed to a secure website where they provided electronic informed consent for participation. Participants were then asked questions about demographics, including sex, 
age, ethnicity, occupation, marital status, current psychopharmacological or psychological treatment, and involvement in current litigation. Next, they were asked to complete the RBQ, P4, and LEC-5. To be eligible for the study, and in line with previous research on retrospective bullying experiences (Blood \& Blood, 2016; Schäfer et al., 2004), participants had to endorse a history of bullying victimization on the RBQ, either during their primary or secondary education or more recently in a work or educational setting. They also had to endorse psychological distress directly related to the bullying victimization experience as indicated by items 32,33 , and $35-40$ in section 3 on the RBQ (see Appendix A). Individuals were ineligible to participate if the indicated that they: (a) were currently engaged in psychotherapy for exposure to bullying, (b) under 18 years of age, (c) had changes in their pharmacological treatment in the past six weeks or were going to have changes during the course of the trial, (d) were currently hospitalized or would be hospitalized during the course of the trial, (e) were taking antipsychotic medication, (f) had substance related problems, (g) were involved in litigation related to their bullying victimization, (h) were at higher risk for suicide (determined by scores on the P4 screener), or (i) were unwilling to provide emergency contact information. All interested individuals, whether eligible or ineligible for participation, were provided with contact information for emergencies (e.g., suicide ideation), including a phone number for the Mobile Crisis Services in Saskatchewan. Ineligible participants were provided with contact information for mental health care providers in the province or for national mental health organizations if they were not located in Saskatchewan.

2.6.2. Baseline measurement. Participants who meet the eligibility criteria for participation in the trial were directed to complete the self-report questionnaires 
described above. Participants in all groups were asked to complete the SIPS, DASS-21, and DAR-5 weekly for the duration of the trial.

2.6.3. Randomization. Following completion of the screening measures, participants were randomized into one of three study conditions. Group assignment was determined using a randomization plan generator (http://www.randomization.com), in line with the Consolidated Standards of Reporting Trials guidelines (CONSORT; Schulz, Altman, \& Moher, 2010). Blocked randomization with randomly selected block sizes was used to reduce the risk of bias in group allocation (Efird, 2010). Participants were notified, prior to the randomization, that they might be assigned to the waitlist and that, upon completion of the waitlist period, they would be eligible to partake in the CPT or SM protocols. Once randomized into a group, participants were emailed a link to the baseline measures.

\subsubsection{Experimental phase}

2.6.4.1. CPT group. The participants in the CPT group had access to 12 textbased modules of therapy (see Appendix S). Two sessions became available each week. The reading level of text was tested using the readability statistics option in Microsoft Word, which determined that the level was approximately eighth grade (i.e., 12 -14 years old). The treatment was therapist-guided and conducted on the internet through the secure web server of the University of Regina Online Therapy Unit (https://www.onlinetherapyuser.ca/). The therapist was the primary investigator, a senior doctoral student in Clinical Psychology with comprehensive training in CPT for PTSD and other trauma-focused treatments, supervised by a Registered Doctoral Psychologist with extensive experience in clinical supervision, research and practice. Supervision was 
scheduled on approximately weekly basis and provided as needed between these scheduled meetings. Therapist contact involved once a week approximately 30-50 minute long review of client progress (e.g., progress through modules, psychodeducation, encouragement to continue practicing the skills covered in treatment), feedback on the homework assignments (e.g., task reinforcement, “well done!”), and alliance building (e.g., empathetic statements, "I can only image how difficult this must have been for you”) using secure email (Schneider, Hadjistavropoulos, \& Faller, 2016). Participants were also encouraged to ask questions regarding the content of the treatment modules and homework assignments as needed using the secure email or regular email, which the therapist answered with brief messages approximately twice per week. Participants who missed a session and had not logged into their account on the web server were sent a reminder through regular email. Participants who missed two and three sessions were sent reminders that included psychoeducation about the counterintuitive association between avoidance behaviours and psychological distress. Participants who missed three sessions or more, and did not respond to emails, were called on the phone and voice mail messages left if needed. Every second session (i.e., once per week) participants were asked to complete three disorder-specific symptom measures (i.e., SIPS, DASS-21, and DAR-5). Treatment followed the protocol of Resick et al. (2014), with adjustments in wording to fit the social trauma and bullying victimization framework.

Session one focused on psychoeducation about the relationship between cognitions, behaviours, and emotions as well as social trauma, PTSD symptoms, stuck points (i.e., beliefs interfering with recovery from the bullying victimization), and treatment rationale. For homework, participants were asked to write an impact statement 
that outlined the meaning of the bullying victimization experience and why they believed the victimization occurred. This statement was reviewed by the therapist and used as a reference for identifying stuck points in following sessions. Session two centered around the meaning of the bullying victimization. Participants read about stuck points (i.e., assimilation, over-accommodation) and attempted to identify them in their impact statement. Participants also learned to fill out Antecedent, Behaviour, and Consequence (ABC) sheets that addressed dysfunctional relationships between thoughts, emotions, and behaviours. For homework, participants were asked to complete ABC sheets. Session three focused on further differentiating between thoughts and feelings. The participants reviewed therapist feedback and learned about thought challenging and the use of Socratic questions (e.g., "What else could we assume?"). Homework assignments included writing a trauma account of a specific bullying victimization experience identified as most distressing. For homework, participants were instructed to read their trauma account daily and complete ABC sheets. During Session four, participants rewrote their trauma account with emphasis on incorporating emotions into the description. Participants were then asked to read the trauma account aloud and instructed to experience the emotions without distraction or avoidance. Analogies such as the "shaken soda bottle" analogy (i.e., "Natural emotions can be viewed like shaken soda bottles. When the cap comes off the bottle, there is a rush, but it is temporary and eventually the soda flattens"; Resick et al., 2014, p. 72) were provided for encouragement. Participants also begun to consider contextual factors in the bullying victimization (e.g., "I could not defend myself because was I so young"). For homework, participants rewrote their trauma account and completed daily ABC sheets. Session five centered around writing 
another trauma account and identifying stuck points. Participants received feedback about their homework from Session four and were instructed to read their trauma accounts aloud and write down how they perceived this time from the previous time. As homework, they were provided with a Challenging Questions worksheet and asked to challenge at least one stuck point per day. In Session six, participants continued working on challenging stuck points, and faulty thinking patterns (i.e., habitual patterns of maladaptive thoughts, such as jumping to conclusions, and oversimplifying) were introduced. For homework, participants were asked to record faulty thinking patterns. Sessions seven to 11 focused on identifying common dysfunctional trauma-related themes in cognition. Homework for these sessions involved completing the Challenging Questions worksheets. According to Resick et al. (2014), the common dysfunctional themes associated with traumatization include issues with: (a) safety (e.g., "All people are dangerous"), which may lead to social isolation and loneliness, (b) trust (e.g., "I have bad judgment about people"), which may lead to self-doubt, (c) power/control (e.g., "I am helpless"), which may lead to self-destructive behaviours and hopelessness, (d) intimacy (e.g., "I am incapable of being close to someone"), which may lead to emptiness and failure to experience connectedness, and (e) esteem (e.g., "I am basically flawed"), which may lead to depression and shame. Session 12 focused on treatment termination, including brief review of skills, reflection of progress in the treatment, and relapse prevention.

2.6.4.2. SM group. Participants randomized into the SM group had access to 12 text-based modules that covered behavioural stress reduction strategies (see Appendix T; e.g., Gardner, Rose, Mason, Tyler, \& Cushway, 2005; Varvogli \& Darviri, 2011). In 
order not to confound with CPT, cognitive strategies for stress relief were omitted from the SM protocol. The reading level of text was tested using the readability statistics option in Microsoft Word, which determined that the level was approximately eighth grade (i.e., 12 -14 years old). The intervention was therapist-guided and conducted on the internet through the secure web server of the University of Regina Online Therapy Unit. The therapist was the primary investigator, a senior doctoral student in Clinical Psychology with comprehensive training in CPT and other trauma-focused treatments, supervised by a Registered Doctoral Psychologist with extensive experience in clinical supervision, research, and treatment. Supervision was scheduled on approximately weekly basis and provided as needed between these scheduled meetings. Therapist contact involved once a week approximately 30-50 minute long review of client progress (e.g., progress through modules, psychodeducation, encouragement to continue practicing the skills covered in treatment), feedback on the homework assignments (e.g., task reinforcement, “well done!”), and alliance building (e.g., empathetic statements, “I can only image how difficult this must have been for you") using secure email (Schneider, Hadjistavropoulos, \& Faller, 2016). Participants were also encouraged to ask questions regarding the content of the treatment modules and homework assignments as needed using the secure email or regular email, which the therapist answered with brief messages approximately twice per week. Participants who missed a session and had not logged into their account on the web server were sent a reminder through regular email. Participants who missed two and three sessions were sent reminders that included psychoeducation about the counterintuitive association between avoidance behaviours and psychological distress. Participants who missed three sessions or more, and did not respond to emails, 
were called on the phone and voice mail messages left if needed. Every second session (i.e., once per week) participants were asked to complete three disorder-specific symptom measures (i.e., SIPS, DASS-21, and DAR-5). The SM protocol was structured for the purpose of this trial.

The two major goals of the SM intervention were to facilitate effective coping with stress and increase general well-being. Session one provided an overview of the SM intervention and psychoeducation about bullying, the physiological stress system, and impact of stress on health and daily functioning. For homework, participants were asked to monitor and record their stressors (e.g., situation) and physical stress responses. Session two introduced diaphragmatic breathing (i.e., using breathing to relax and reduce stress). For homework, participants were asked to engage in daily breathing exercises. Session three introduced guided imagery for relaxation. Homework included engaging in daily breathing exercises while imagining a relaxing scenery. Session four provided psychoeducation about soothing activities involving the five senses (i.e., seeing, hearing, smelling, touching, tasting). Participants were provided with a list of soothing activities and were asked to complete one activity using each of the senses per day for homework. Additionally, participants were encouraged to continue their daily breathing exercises between sessions. Sessions five and six taught progressive muscle relaxation (PMR) and participants practiced daily PMR exercises for homework. Session seven introduced emotion-focused and problem-focused coping for stress management. For homework, participants were asked to record their stress coping strategies. Sessions 8-11 focused on psychoeducation about stress reduction in everyday life (e.g., engaging in pleasant activities, spending time in nature, social contact). Participants were asked to identify 
goals for stress reduction in their day-to-day activities (e.g., exercise, eat healthy, socialise) and continue daily PMR exercises for homework. Session 12 centered around treatment termination, and included a brief review of the skills covered in program, reflection of client progress, and relapse prevention.

2.6.4.3. Waitlist group. Participants randomized into the waitlist group were sent a Qualtrics link through email to complete the weekly measures (i.e., SIPS, DASS-21, and DAR-5) for six consecutive weeks. No therapist contact was provided during the waitlist period. Following completion of the waitlist, the participants were invited to be re-randomized into either CPT or SM following the same protocols described above.

2.6.5. Follow-up. Participants were emailed a Qualtrics link to complete the follow-up measures one month after completion of therapy. Each follow-up assessment consisted of the primary and secondary measures administered at baseline and posttreatment.

\subsection{Statistical analysis}

Primary analyses were conducted using HLM. HLM analyzes outcomes at each time point (level 1) nested within individuals (level 2) and group outcomes as a function of both characteristics of the group and group members. The advantages of using hierarchical analyses over liner regression with data from randomized controlled trials include more accuracy in the estimation and that the analysis can handle missing data (Anderson, 2012; Woltman, Feldstain, MacKay, \& Rocchi, 2012). The current trial employed intention-to-treat approach to the analyses in line with the CONSORT guidelines for randomized controlled trials (Schulz, Altman \& Moher, 2010). Participants had to have completed at least one treatment session in CPT and SM and at least one 
measurement occasion in the waitlist to be included in the analyses. Proportion of trial participants that moved from the clinically significant range on the symptom measures (i.e., total scores indicating symptom levels of clinically significant difficulties) from baseline to post-treatment and one month follow-up were calculated using complete cases. The percentage of missing data at post-treatment and follow-up was approximately 50$70 \%$ due to participant drop out and the intent-to-treat approached used in the trial. Using multiple imputation to calculate the missing values was, therefore, not appropriate (Jacobsen, Gluud, Wetterslev, \& Winkel, 2017). The disadvantages of using complete cases to calculate the percentage of participants that no longer had clinically significant symptoms on the self-report measures include that the small data available severely undermines the conclusion and generalizability of the calculation. However, missing data did not affect the HLM outcome testing (Anderson, 2012). HLM handles missing data in outcome measures by using several unfixed data points for each participant (i.e., does not rely on an assumption that measurements occasions are fixed and equal in number) and with weighted estimates that combine both measurements from participants and the sample (Bauer, Lambert, \& Nielsen, 2004).

Hypotheses 1 and 2 were tested using HLM of the effects of group, time, number of treatment sessions completed, time of bullying victimization experience (i.e., remote or recent), and number of bullying victimization experiences (i.e., one or more), and group-by-time on each of the dependent variables. Effects of group-by-time and number of treatment sessions completed were expected to be significant, indicating changes in the dependent variables over time as a function of group assignment. Time of bullying victimization experiences was expected to be a significant predictor, with recent bullying 
victimization experiences associated with stronger maladaptive appraisals and higher psychological distress compared to remote bullying victimization. Similarly, number of bullying victimization experiences was expected to be significant predictor, with more than one bullying victimization experience associated with stronger maladaptive appraisals and higher psychological distress compared to a single bullying victimization experience.

Hypotheses were tested using two-tailed tests with $\alpha=.05$. Models were computed using maximum likelihood estimation and included both fixed and random effects to account for possible baseline differences in measurement scores. Time was treated as a repeated effect and a covariant to account for within-subject correlations in residual errors arising from each participant completing the same questionnaires at each measurement occasion. Models were rerun when time was a significant covariant with a categorical dummy time variable to test differences in the dependent variable scores between measurement occasions. All models were tested using both variance components and unstructured variance-covariance-matrix and the difference in model fit based on using each covariance structure was examined using the likelihood ratio chi-square test. Model fit was further assessed by examining the Akaike Information Criterion, and the Bayesian Information Criterion, with lower values indicating an improvement in fit (Tabachnick \& Fidell, 2013). Effect sizes for mean changes in outcome variables were calculated using standardized mean differences (Cohen's $d$ ).

Secondary analyses were conducted using multiple regressions. Hypothesis 3 was tested in line with the four steps of mediation proposed by Baron and Kenny (1986). First, a simple regression analysis was conducted to test the direct effect of the independent 
variable (i.e., level of perceived event centrality) on the dependent variable (i.e., strength of maladaptive appraisals). Second, a simple regression tested the effect of the independent variable (i.e., level of perceived event centrality) on each of the mediator variables (i.e., level of resilience, perceived social support, and coping styles). Third, simple regressions tested the effect of each of the mediator variables on the dependent variable. Fourth, a multiple regression was conducted where the independent variable and mediator variables predicted the dependent variable. The interaction effect of perceived event centrality and each of the mediators on strength of maladaptive appraisals was expected to be larger than the direct effect of trauma centrality alone on maladaptive appraisals. Hypothesis 4 was tested using moderator regression. First, a simple linear regression examined whether the independent variable (i.e., strength of maladaptive appraisals) significantly predicted the dependent variable (i.e., psychological distress measured by PCL-5, SIPS, DAR-5, and DASS-21 scores). Second, a bivariate correlation analysis was used to examine the strength of correlation between the moderator variable (i.e., number of bullying victimization experiences) and independent variable (i.e., maladaptive appraisals). Third multiple regression was conducted to test the effect of the moderator variable on the relationship between the independent and dependent variables. The combined effect of strength of maladaptive appraisals and number of bullying victimization experiences on psychological distress was expected to be stronger than the effect of maladaptive appraisals on psychological distress alone. 


\section{RESULTS}

\subsection{Descriptive Statistics}

Of the 202 individuals who accessed the pre-screening questionnaires, 38 did not meet the eligibility criteria. Seventeen individuals did not reside in Saskatchewan, seven endorsed a change in their psychotropic medication and were invited to participate in the trial once six weeks had passed from the medication change, three indicated that they were already in psychological treatment focusing on the consequences of exposure to bullying, five reported that they were involved in a litigation directly related to their bullying experience at work, one endorsed not feeling bothered by their bullying experience, and one endorsed not having regular access to a computer or internet. Additionally, four individuals were deemed ineligible because they reported a current episode of mania or psychosis and high suicidal ideation. Phone calls were placed to these four individuals, as per the informed consent which indicated that current risk of harm to self and others would be evaluate through telephone if high risk was indicated on the screening measures (see above). All ineligible individuals were provided with contact information for mental health and crisis services either in Saskatchewan or their respective Canadian province or, in one case, internationally.

A total of 105 participants were eligible for participation in the trial, 35 were randomized into each of the study groups (Figure 2). Of the 30 participants randomized into CPT, 20 completed the treatment, resulting in an attrition rate of $33 \%$. The attrition rate in the SM group was 53\%, with 30 participants randomized into SM and 14 completing the treatment program. The majority of participants in CPT and SM who dropped out of treatment did so before before session three. The attrition rate in the 
waitlist was $40 \%$, with 30 participants randomized and 18 completing the waiting period. Demographics were similar across study groups, and similar for treatment completers and non-completers. A greater number of participants in the trial identified as women (67.80\%), compared to other genders, and majority of participants were Caucasian, married or common law, and were employed full-time. The mean age of participants was 43.71 years $(S D=11.32)$. No differences were found between completers and noncompleters' age $\left(\chi^{2}(34, N=87)=31.78, p=.588\right)$. An overview of the demographics in the sample at baseline is presented in Table 1.

Bullying victimization experiences early in life that left a lasting negative impact were more common in the sample $(75.9 \%)$ than recent bullying victimization experiences. Slightly more participants reported experiencing a single instance of bullying victimization $(56.9 \%)$ in their life-time compared to multiple instances. The majority of participants indicated that their bullying experiences had been victimization only (76.90\%), as opposed to involvement in bullying as both a perpetrator and a victim. All participants in the current trial reported exposure to trauma as measured by the LEC-5. Table 4 presents an overview of the types of bullying experiences endorsed by participants and accumulated information about trauma exposure.

Clinically significant elevations were found on all symptoms measures at baseline. Majority of participants (56.1\%) endorsed clinically significant PTSD symptoms in relation to their bullying victimization experience on the PCL-5 (i.e., a total score of 30 or higher). Maladaptive appraisals associated with perceived trauma from bullying victimization and symptoms of PTSD (i.e., a total score of 133 or higher on the PTCI) were also prevalent in the sample (59.6\%). The majority of participants $(61.0 \%)$ reported 
clinically significant symptoms of social anxiety as measured by the SIPS (i.e., a cut-off total score of 21). On the DASS-21 Depression subscale, $22.1 \%$ of the sample reported experiencing symptoms of depression of moderate severity at baseline, $13.1 \%$ severe symptoms, and $15.3 \%$ extremely severe symptoms. Similarly, on the DASS-21 Anxiety subscale, $23.8 \%$ of participants reported moderate anxiety, $6.8 \%$ severe anxiety, and $20.4 \%$ extremely severe anxiety. On the DASS-21 Stress subscale $20.4 \%$ participants reported moderate stress at baseline, $17.0 \%$ severe stress, and $8.5 \%$ extremely severe stress. On the DAR-5, 39.1\% of participants reported experiencing frequent, intense and long lasting anger, and aggressive responses interfering with functioning (i.e., a total score of 12 or higher). On the DTS, mean total scores across all groups were in the lower to moderate range (i.e., range of total scores from 1-5), indicating low to moderate ability to tolerate distress. Mean total and subscale scores on the ASI-3 indicated moderate anxiety sensitivity (i.e., in a range of total scores from 0-72, and subscale scores from 024) across the all groups. On the IUS-12, total mean scores indicated high levels of IU (i.e., in a range of scores from 12-60) and moderate to high levels of Prospective IU (i.e., in a range of total scores from 7-35) and Inhibitory IU (i.e., in a range of total scores from 5-25). Correlation between the outcome measures varied from moderate to high (see Table 3). Mean total scores on the CES suggested that event centrality of the bullying victimization experience was at the higher end in the range of total scores (i.e., in a range from 7-35) across all group. On the CERQ, total mean scores indicated there were higher tendencies in the sample for engaging in acceptance, rumination, positive refocusing, and planning to regulate emotions (i.e., range of total scores from 2-10) compared to the other cognitive emotion regulation styles. On the BRS, total mean scores suggested that the 
level of resilience in the sample was low (i.e., total scores from 1-2.99). Finally, on the MSPSS, the level of perceived social support was moderate across all groups; but, total scores were slightly higher for perceived social support from significant others (i.e., range of total scores from 1-7). The mean total score on the MSPSS also indicated that global satisfaction with social support in the sample was in the moderate range. Total mean scores at baseline on all of self-report questionnaires included in the trial are presented in Table 2 .

Session-by-session changes in mean SIPS, DASS-21, and DAR-5 scores for treatment completers in CPT and SM are depicted in Figures 3 and 4. Total scores were standardized to account for differences in scaling and for easier interpretation of the graphs. The linear graphs show that symptoms of social anxiety, depression, anxiety and stress, and anger gradually decrease over time in both treatment groups. Decreases in symptoms were, however, steeper and more linear in SM over time, compared to CPT, where changes occurred at a slower rate and symptoms increased before beginning to decrease.

\subsection{Acceptability Testing}

The content surveys were emailed to the 90 treatment completers and noncompleters who had been randomized to either CPT or SM. Twenty-two participants completed the survey. Thereof, 11 participants had been randomized to CPT. Five responders indicated that they had completed 12 internet-delivered sessions of CPT, one had completed 11 sessions, one two sessions, one a single session, and three reported they had dropped-out of the trial before completing at least one sessions. The three participants who dropped out of the trial before completing at least one session were 
identified as non-completers, in line with the intention-to-treat criteria for this study. The reasons provided for discontinuing participation included being too occupied at work, and preferring in-person treatment.

Of the six participants who completed 11 and 12 sessions of CPT and were able to provide feedback about the content of treatment, three $(50.0 \%)$ indicated they strongly agreed that the treatment was helpful and three $(50.0 \%)$ indicated they agreed that the treatment was helpful. The impact statement covered in the first two sessions, the trauma account in sessions three and four, the ABC worksheets and challenging beliefs worksheets incorporated into most sessions, and discussion of power/control issues in session nine, were deemed most helpful. Two of the six participants reported finding the length of treatment program adequate $(33.3 \%)$, while four reported finding the program slightly too short (66.7\%), and five participants strongly agreed that therapist communication was helpful (83.3\%) and one somewhat agreed (16.7\%). All six participants reported that they had worked hard in the CPT treatment, practiced the skills covered in treatment (i.e., definitely true $66.7 \%$, probably true $33.3 \%$ ), used the skills in their everyday life (i.e., a great deal $33.30 \%$, a lot 50.0\%, a moderate amount $16.7 \%$ ), and that they would recommend this program (i.e., strongly agree $83.3 \%$, agree $16.7 \%$ ). Five of the six participants identified that their beliefs about their bullying experience changed in helpful ways from participating in the program (i.e., strongly agree $33.3 \%$, agree $50.0 \%$ ) but one participant was unsure (i.e., neither agree or disagree $16.7 \%$ ). All six participants reported that their beliefs about themselves and other people changed in helpful ways from participating in the CPT program (i.e., agree 50\%, somewhat agree $50 \%)$. 
Eleven responders to the content survey had been randomized into the SM condition. Thereof, four reported completing 12 internet-delivered sessions, one four sessions, one three sessions, and one two sessions. Four participants indicated that they dropped out of the trial before completing at least one session of SM (i.e., noncompleters), reasoning that the internet format of the program had not been suitable and that they were too busy with work to participate in the study. The participant who indicated dropping out after two SM sessions, discontinued because they did not find the program helpful. The two participants who discontinued SM after three and four sessions explained they had found the duration of the program slightly too long.

Of the four participants who completed 12 sessions of SM and were able to comment on session content, all indicated finding the treatment helpful (i.e., agree $100.0 \%$ ) and that they would recommend it to others (i.e., agree, $50.0 \%$ and somewhat agree, 50.0\%). Identifying stressors and stress responses and diaphragmatic breathing covered in sessions one and two and applied throughout the program were identified as the most helpful, followed by the five senses practice in session four. Three of the four participants indicated that the program length was adequate (75.0\%), and one that it was too long $(25.0 \%)$. All four participants indicated that they worked hard in the program and practiced the skills covered in sessions (100.0\%), and that communication with the therapist was helpful (i.e., strongly agree, 100.0\%). The four SM participants further reported using the strategies covered in sessions in their everyday life (i.e., a lot 50.0\%, a moderate amount $50.0 \%$ ). One participant indicated that their beliefs about their bullying experience changed in helpful ways as a result of completing SM (i.e., agree, 50.0\%) and two indicated that their beliefs did not change at all (i.e., strongly disagree, 50.0\%, 
somewhat disagree 50.0\%). Three of the four participants indicated that their beliefs about themselves and other people changed in helpful was from participating in the program (i.e., agree, $75.0 \%$ ), and one that their beliefs stayed the same (i.e., disagree, $25.0 \%)$ 
Table 1. Demographics at baseline

\begin{tabular}{|c|c|c|c|}
\hline & $\begin{array}{c}\text { Total Sample } \\
(\mathrm{n}=90)\end{array}$ & $\begin{array}{c}\text { Completers } \\
(\mathrm{n}=52)\end{array}$ & $\begin{array}{c}\text { Non-Completers } \\
(\mathrm{n}=\mathbf{3 8})\end{array}$ \\
\hline & $N(\%)$ or $M(S D)$ & $N(\%)$ or $M(S D)$ & $N(\%)$ or $M(S D)$ \\
\hline \multicolumn{4}{|l|}{ Gender } \\
\hline Male & $27(30.00 \%)$ & $15(28.80 \%)$ & $12(31.60 \%)$ \\
\hline Female & $61(67.80 \%)$ & $36(69.20 \%)$ & $25(65.80 \%)$ \\
\hline Transgender & $2(2.20 \%)$ & $1(1.9 \%)$ & $1(2.60 \%)$ \\
\hline Age (years) & $43.71(11.32)$ & $43.37(12.47)$ & $44.16(9.80)$ \\
\hline \multicolumn{4}{|l|}{ Ethnicity } \\
\hline African & $0(0.00 \%)$ & $0(0.00 \%)$ & $0(0.00 \%)$ \\
\hline Asian & $1(1.10 \%)$ & $1(1.90 \%)$ & $0(0.00 \%)$ \\
\hline Caucasian & $85(94.40 \%)$ & $49(94.20 \%)$ & $36(94.70 \%)$ \\
\hline Indigenous & $1(1.10 \%)$ & $0(0.00 \%)$ & $1(2.6 \%)$ \\
\hline Metis & $2(2.20 \%)$ & $1(1.9 \%)$ & $1(2.6 \%)$ \\
\hline Hispanic & $0(0.00 \%)$ & $0(0.00 \%)$ & $0(0.00 \%)$ \\
\hline Latino & $0(0.00 \%)$ & $0(0.00 \%)$ & $0(0.00 \%)$ \\
\hline Multiracial & $0(0.00 \%)$ & $0(0.00 \%)$ & $0(0.00 \%)$ \\
\hline South-Asian & $0(0.00 \%)$ & $0(0.00 \%)$ & $0(0.00 \%)$ \\
\hline Not listed & $0(0.00 \%)$ & $0(0.00 \%)$ & $0(0.00 \%)$ \\
\hline Prefer not to answer & $1(1.10 \%)$ & $1(1.9 \%)$ & $0(0.00 \%)$ \\
\hline \multicolumn{4}{|l|}{ Relationship Status } \\
\hline Single & $26(28.90 \%)$ & $14(26.90 \%)$ & $12(31.60 \%)$ \\
\hline Committed & $10(11.10 \%)$ & $5(9.60 \%)$ & $5(13.20 \%)$ \\
\hline relationship & $48(53.30 \%)$ & $28(53.80 \%)$ & $20(52.60 \%)$ \\
\hline Married/common law & $4(4.40 \%)$ & $3(5.80 \%)$ & $1(2.60 \%)$ \\
\hline Divorced & $0(0.00 \%)$ & $0(0.00 \%)$ & $0(0.00 \%)$ \\
\hline Not listed & $2(2.20 \%)$ & $2(3.80 \%)$ & $0(0.00 \%)$ \\
\hline Prefer not to answer & & & \\
\hline \multicolumn{4}{|l|}{ Highest Education } \\
\hline High school & $8(8.90 \%)$ & $4(7.70 \%)$ & $4(10.50 \%)$ \\
\hline High school equivalent & $2(2.20 \%)$ & $1(1.90 \%)$ & $1(2.60 \%)$ \\
\hline College diploma & $28(31.10 \%)$ & $12(23.10 \%)$ & $16(42.10 \%)$ \\
\hline Undergraduate degree & $34(37.80 \%)$ & $22(42.30 \%)$ & $12(31.60 \%)$ \\
\hline Master's degree & $13(14.40 \%)$ & $8(15.40 \%)$ & $0(0.00 \%)$ \\
\hline Doctoral degree & $1(1.1 \%)$ & $1(1.9 \%)$ & $0(0.00 \%)$ \\
\hline Not listed & $3(3.3 \%)$ & $3(5.8 \%)$ & $0(0.00 \%)$ \\
\hline Prefer not to answer & $1(1.1 \%)$ & $1(1.9 \%)$ & $0(0.00 \%)$ \\
\hline \multicolumn{4}{|l|}{ Employment } \\
\hline Employed full-time & $56(62.22 \%)$ & $32(61.50 \%)$ & $24(63.20 \%)$ \\
\hline Employed part-time & $6(6.67 \%)$ & $4(7.70 \%)$ & $2(5.30 \%)$ \\
\hline Unemployed & $7(7.78 \%)$ & $4(7.70 \%)$ & $3(7.90 \%)$ \\
\hline Homemaker & $1(1.11 \%)$ & $0(0.00 \%)$ & $1(2.60 \%)$ \\
\hline Student full-time & $4(4.44 \%)$ & $1(1.90 \%)$ & $3(7.90 \%)$ \\
\hline Student part-time & $0(0.00 \%)$ & $0(0.00 \%)$ & $0(0.00 \%)$ \\
\hline Retired & $4(4.44 \%)$ & $3(5.80 \%)$ & $1(2.60 \%)$ \\
\hline On a medical leave & $3(3.33 \%)$ & $2(3.80 \%)$ & $1(2.60 \%)$ \\
\hline
\end{tabular}


On disability

Not listed

Prefer not to answer

\section{Household Income}

Less than $\$ 10,000$

\$10,000-\$19,999

\$20,000-\$29,999

$\$ 30,000-\$ 39,999$

$\$ 40,000-\$ 49,999$

$\$ 50,000-\$ 59,999$

\$60,000-\$69,999

$\$ 70,000-\$ 79,999$

$\$ 80,000-\$ 89,999$

$\$ 90,000-\$ 99,999$

\$100,000-\$149,999

More than $\$ 150,000$

Prefer not to answer
$2(2.22 \%)$

$5(5.56 \%)$

$2(2.22 \%)$

$5(5.60 \%)$

$3(3.30 \%)$

$5(5.60 \%)$

$1(1.10 \%)$

$3(3.30 \%)$

$8(8.90 \%)$

$8(8.90 \%)$

$6(6.70 \%)$

$0(0.00 \%)$

$2(2.20 \%)$

$19(21.10 \%)$

$13(14.40 \%)$

$17(18.90 \%)$

$$
\begin{gathered}
2(3.80 \%) \\
3(5.80 \%) \\
1(1.90 \%)
\end{gathered}
$$

$4(7.70 \%)$

$3(5.80 \%)$

$2(3.80 \%)$

$1(1.90 \%)$

$2(3.80 \%)$

$5(9.60 \%)$

$2(3.80 \%)$

$2(3.80 \%)$

$0(0.00 \%)$

$1(1.90 \%)$

$10(19.20 \%)$

$9(17.30 \%)$

$11(21.20 \%)$
$0(0.00 \%)$

$2(5.30 \%)$

$1(2.60 \%)$

$1(2.60 \%)$

$0(0.00 \%)$

$3(7.90 \%)$

$0(0.00 \%)$

$1(2.60 \%)$

$3(7.90 \%)$

$6(15.80 \%)$

$4(10.50 \%)$

$0(0.00 \%)$

$1(2.60 \%)$

$9(23.70 \%)$

$4(10.50 \%)$

$6(15.80 \%)$ 
Table 2. Self-reports at baseline by trial group

\begin{tabular}{|c|c|c|c|c|c|}
\hline $\begin{array}{l}\text { Self-report at } \\
\text { baseline }\end{array}$ & $\begin{array}{c}\text { Total } \\
\text { Sample } \\
(n=52)\end{array}$ & $\begin{array}{c}\text { CPT } \\
(n=20)\end{array}$ & $\begin{array}{c}\text { SM } \\
(n=14)\end{array}$ & $\begin{array}{c}\text { WL } \\
(n=18)\end{array}$ & $\begin{array}{c}\text { Reliability } \\
\text { Chronbach's } \\
\text { alpa }\end{array}$ \\
\hline \multicolumn{6}{|l|}{ Outcomes } \\
\hline PTCI total & $124.39(32.31)$ & $124.57(36.05)$ & $117.78(31.17)$ & $131.56(27.96)$ & .96 \\
\hline PTCI NSB & $70.37(23.83)$ & $69.70(29.96)$ & $67.00(20.45)$ & $75.13(23.28)$ & .95 \\
\hline PTCI NOB & $34.40(8.13)$ & $35.70(8.01)$ & $31.22(9.23)$ & $36.13(6.16)$ & .93 \\
\hline PTCI SB & $19.62(6.31)$ & $19.17(6.79)$ & $19.56(7.07)$ & $20.31(4.87)$ & .76 \\
\hline PCL-5 total & $33.63(16.58)$ & $32.09(18.00)$ & $29.83(14.11)$ & $40.13(16.09)$ & .96 \\
\hline SIPS total & $21.69(13.57)$ & $20.83(14.36)$ & $19.55(12.77)$ & $25.63(13.35)$ & .96 \\
\hline SIPS SIA & $10.66(6.07)$ & $10.17(6.91)$ & $10.65(5.70)$ & $11.38(5.52)$ & .97 \\
\hline SIPS FOE & $6.97(5.90)$ & $7.04(5.97)$ & $5.20(5.47)$ & $9.06(5.94)$ & .94 \\
\hline SIPS FAA & $4.07(3.12)$ & $3.61(3.17)$ & $3.70(3.03)$ & $5.19(3.06)$ & .80 \\
\hline DASS-21 total & $21.90(13.46)$ & $20.91(13.35)$ & $19.15(14.40)$ & $26.75(11.80)$ & .95 \\
\hline DASS D & $7.59(5.49)$ & $7.48(5.37)$ & $6.25(5.50)$ & $9.44(5.90)$ & .94 \\
\hline DASS A & $5.29(4.67)$ & $4.96(5.20)$ & $4.65(4.70)$ & $6.56(3.76)$ & .84 \\
\hline DASS S & $9.02(5.15)$ & 8.48 (4.79) & $8.25(5.70)$ & $10.75(4.82)$ & .91 \\
\hline DAR-5 total & $10.88(4.42)$ & $15.21(4.86)$ & $10.21(4.55)$ & $12.06(5.25)$ & .87 \\
\hline DTS total & $2.78(1.00)$ & $3.05(1.05)$ & $2.82(.97)$ & $2.35(.85)$ & .93 \\
\hline DTS AB & $2.47(1.29)$ & $2.62(1.44)$ & $2.44(1.07)$ & $2.93(1.33)$ & .88 \\
\hline DTS AP & $2.89(1.15)$ & $3.01(2.40)$ & $3.02(1.08)$ & $2.56(1.16)$ & .89 \\
\hline DTS R & $2.89(1.15)$ & $3.00(1.22)$ & $3.19(1.06)$ & $1.20(1.02)$ & .71 \\
\hline DTS T & $2.78(1.21)$ & $2.74(1.24)$ & $1.36(1.14)$ & $2.92(1.33)$ & .86 \\
\hline ASI-3 total & $40.21(13.67)$ & $37.83(11.63)$ & $39.00(11.68)$ & $45.00(17.60)$ & .92 \\
\hline ASI PHY & $12.25(5.80)$ & $11.35(5.81)$ & $12.00(5.04)$ & $13.81(6.58)$ & .90 \\
\hline ASI COG & $11.19(4.73)$ & $10.13(3.94)$ & $10.61(3.48)$ & $13.38(6.30)$ & .86 \\
\hline ASI SOC & $16.77(6.20)$ & $16.35(5.52)$ & $16.39(5.60)$ & $17.81(7.50)$ & .89 \\
\hline IUS-12 total & $36.44(12.21)$ & $37.13(12.62)$ & $32.72(11.25)$ & $39.63(12.30)$ & .96 \\
\hline IUS P & $22.83(7.39)$ & $23.09(7.63)$ & $20.67(7.02)$ & $24.88(7.23)$ & .94 \\
\hline IUS I & $13.62(5.28)$ & $14.04(5.64)$ & $12.06(4.57)$ & $14.75(5.39)$ & .94 \\
\hline \multicolumn{6}{|l|}{ Secondary } \\
\hline CES total & $28.86(8.75)$ & $27.91(10.11)$ & 27.17 (6.99) & $32.13(8.02)$ & .93 \\
\hline CERQ total & $58.76(8.42)$ & $57.04(9.61)$ & $58.56(8.96)$ & $61.44(5.07)$ & .71 \\
\hline CERQ SB & $5.74(2.34)$ & $5.44(2.41)$ & $5.56(2.75)$ & $6.38(1.67)$ & .94 \\
\hline CERQ AC & $7.60(2.06)$ & $7.22(2.59)$ & 7.78 (1.99) & $7.94(1.06)$ & .91 \\
\hline CERQ RU & $6.95(2.31)$ & $6.52(2.43)$ & $7.06(2.71)$ & $7.44(1.55)$ & .81 \\
\hline CERQ PR & $5.46(2.19)$ & $5.35(2.08)$ & $5.89(2.45)$ & $5.13(2.09)$ & .87 \\
\hline CERQ PL & $7.19(1.87)$ & $7.31(2.16)$ & $6.89(1.64)$ & $7.38(1.71)$ & .72 \\
\hline CERQ POS & $7.47(1.98)$ & $7.00(2.22)$ & $7.50(1.95)$ & $8.13(1.54)$ & .78 \\
\hline CERQ PIP & $5.56(2.30)$ & $5.44(2.56)$ & $5.56(2.28)$ & $5.75(2.05)$ & .82 \\
\hline CERQ CA & $5.98(2.35)$ & $6.04(2.33)$ & $5.56(2.77)$ & $6.38(1.89)$ & .92 \\
\hline CERQ OB & $6.81(2.49)$ & $6.74(2.43)$ & $6.78(2.46)$ & $6.94(2.75)$ & .92 \\
\hline BRS total & $2.77(.92)$ & $2.89(.93)$ & $2.63(.84)$ & $2.75(1.02)$ & .94 \\
\hline MSPSS total & $3.98(1.43)$ & $4.14(1.37)$ & $4.12(1.43)$ & $3.59(1.53)$ & .95 \\
\hline MSPSS SO & $3.96(1.58)$ & $4.20(1.60)$ & $4.17(1.35)$ & 3.49 (1.78) & .96 \\
\hline MSPSS FA & $3.05(1.55)$ & $3.19(1.52)$ & $3.04(1.67)$ & $2.86(1.52)$ & .95 \\
\hline MSPSS FR & $3.49(1.45)$ & $3.61(1.56)$ & $3.60(1.39)$ & $3.19(1.39)$ & .96 \\
\hline
\end{tabular}

Note: PTCI = Posttraumatic Cognitions Inventory; NSB = Negative Self-Beliefs Scale; NOB = Negative Other-Beliefs Scale; SB = Self-Blame Scale; PCL-5 = Posttraumatic Stress Disorder 
Checklist for DSM-5; SIPS = Social Interaction Phobia Scale; SIA = Social Interaction Anxiety Scale $; \mathrm{FOE}=$ Fear of Overt Evaluations Scale; FAA = Fear of Attracting Attention Scale; DASS$21=$ Depression Anxiety Stress Scales 21; D = Depression Scale; A = Anxiety Scale; S = Stress Scale; DAR-5 = Dimensions of Anger Reactions-5 Scale; DTS = Distress Tolerance Scale; AB = Absorption; AP = Appraisal; $\mathrm{R}=$ Regulation; $\mathrm{T}=$ Tolerance; ASI-3 = Anxiety Sensitivity Index3; $\mathrm{PHY}=$ Physical Concerns COG = Cognitive Concerns; $\mathrm{SOC}=$ Social Concerns; IUS = Intolerance of Uncertainty Scale-Short Form 12; P = Prospective; I = Inhibitory; CES = Centrality of Event Scale-short; CERQ = Cognitive Emotion Regulation Questionnaire-short; SB $=$ Self-Blame; $\mathrm{AC}=$ Acceptance $; \mathrm{RU}=$ Rumination $; \mathrm{PR}=$ Positive Refocusing $; \mathrm{PL}=\mathrm{Planning}$; POS = Positive Reappraisal; PIP = Putting Into Perspective; $\mathrm{CA}=$ Catastrophizing; $\mathrm{OB}=$ Other Blame; BRS = Brief Resilience Scale; MSPSS = Multidimensional Scale of Perceived Social Support; $\mathrm{SO}=$ Significant Other; FA = Family; FR = Friends . 
Table 3. Correlation between outcome measures

\begin{tabular}{llllllll}
\hline & PTCI & PCL-5 & SIPS & DASS-21 & DAR-5 & DTS & IUS-12 \\
\hline PCL-5 & $.77 * * *$ & & & & & & \\
SIPS & $.67 * * *$ & $.59 * * *$ & & & & & \\
DASS-21 & $.70^{* * *}$ & $.80^{* * *}$ & $.49 * * *$ & & & & \\
DAR-5 & $.65^{* * *}$ & $.67 * * *$ & $.45^{* * *}$ & $.61 * * *$ & & & \\
DTS & $.80^{* * *}$ & $.61 * * *$ & $.70^{* * *}$ & $.60 * * *$ & $.57 * * *$ & & \\
IUS-12 & $.74 * * *$ & $.56^{* * *}$ & $.77 * * *$ & $.52^{* * *}$ & $.51 * * *$ & $.71 * * *$ & \\
ASI-3 & $.43^{* * *}$ & $.38^{* * *}$ & $.45^{* * *}$ & $.49 * * *$ & $.41 * * *$ & $.58^{* * *}$ & $.45^{* * *}$ \\
\hline
\end{tabular}

Note: PTCI = Posttraumatic Cognitions Inventory; NSB = Negative Self-Beliefs Scale; NOB = Negative Other-Beliefs Scale; SB = Self-Blame Scale; PCL-5 = Posttraumatic Stress Disorder Checklist for DSM-5; SIPS = Social Interaction Phobia Scale; SIA = Social Interaction Anxiety Scale; FOE = Fear of Overt Evaluations Scale; FAA = Fear of Attracting Attention Scale; DASS21 = Depression Anxiety Stress Scales 21; D = Depression Scale; A = Anxiety Scale; S = Stress Scale; DAR-5 = Dimensions of Anger Reactions-5 Scale; DTS = Distress Tolerance Scale; AB = Absorption; $\mathrm{AP}=$ Appraisal $\mathrm{R}=$ Regulation; $\mathrm{T}=$ Tolerance; ASI-3 = Anxiety Sensitivity Index3; $\mathrm{PHY}=$ Physical Concerns; $\mathrm{COG}=$ Cognitive Concerns; $\mathrm{SOC}=$ Social Concerns; IUS = Intolerance of Uncertainty Scale-Short Form 12; P = Prospective; I = Inhibitory; CES = Centrality of Event Scale-short; 
Table 4. Overview of bullying victimization experiences and exposure to trauma

\begin{tabular}{|c|c|c|c|c|c|c|c|c|}
\hline & \multicolumn{2}{|c|}{$\begin{array}{l}\text { Total Sample } \\
\quad(\mathrm{n}=90)\end{array}$} & \multicolumn{2}{|c|}{$\begin{array}{l}\text { Waitlist } \\
(\mathrm{n}=30)\end{array}$} & \multicolumn{2}{|c|}{$\begin{array}{c}\text { CPT } \\
(\mathrm{n}=30)\end{array}$} & \multicolumn{2}{|c|}{$\begin{array}{c}\text { SM } \\
(n=30)\end{array}$} \\
\hline & $\begin{array}{l}\text { Completers } \\
(\mathrm{n}=52)\end{array}$ & $\begin{array}{c}\text { Non- } \\
\text { Completers } \\
(\mathrm{n}=38)\end{array}$ & $\begin{array}{l}\text { Completers } \\
(\mathrm{n}=18)\end{array}$ & $\begin{array}{c}\text { Non- } \\
\text { Completers } \\
(\mathrm{n}=12)\end{array}$ & $\begin{array}{l}\text { Completers } \\
(\mathrm{n}=20)\end{array}$ & $\begin{array}{c}\text { Non- } \\
\text { Completers } \\
(\mathrm{n}=10)\end{array}$ & $\begin{array}{l}\text { Completers } \\
(\mathrm{n}=14)\end{array}$ & $\begin{array}{c}\text { Non- } \\
\text { Completers } \\
(\mathrm{n}=16)\end{array}$ \\
\hline \multicolumn{9}{|l|}{ Time of BVE } \\
\hline Remote & $39(74.00 \%)$ & $27(71.05 \%)$ & $14(77.80 \%)$ & $9(75.00 \%)$ & $15(75.00 \%$ & $6(60.00 \%)$ & $10(71.43 \%)$ & $12(75.00 \%)$ \\
\hline Recent & $13(25.00 \%)$ & $11(28.95 \%)$ & $4(22.22 \%)$ & $3(25.00 \%)$ & $5(25.00 \%)$ & $4(40.00 \%)$ & $4(28.57 \%)$ & $4(25.00 \%)$ \\
\hline \multicolumn{9}{|l|}{ Number of BVE } \\
\hline One & $29(55.77 \%)$ & $15(39.47 \%)$ & $7(38.89 \%)$ & $4(33.33 \%)$ & $11(55.00 \%)$ & $4(40.00 \%)$ & $11(78.57 \%)$ & $7(43.75 \%)$ \\
\hline More than one & $23(44.23 \%)$ & $23(60.53 \%)$ & $11(61.11 \%)$ & $8(66.67 \%)$ & $9(45.00 \%)$ & $6(60.00 \%)$ & $3(21.43 \%)$ & $9(56.25 \%)$ \\
\hline Victim & $40(76.90 \%$ & $30(78.90 \%)$ & $15(83.30 \%)$ & $9(75.00 \%)$ & $15(75.00 \%)$ & $8(80.00 \%)$ & $10(71.40 \%)$ & $13(81.30 \%)$ \\
\hline Bully-Victim & $12(23.10 \%)$ & $8(21.10 \%)$ & $3(16.70 \%)$ & $3(25.00 \%)$ & $5(25.00 \%)$ & $2(20.00 \%)$ & $4(28.60 \%)$ & $3(18.80 \%)$ \\
\hline \multicolumn{9}{|l|}{ Trauma } \\
\hline Natural Disaster & $19(36.50 \%)$ & $14(36.80 \%)$ & $7(38.90 \%)$ & $4(33.30 \%)$ & $7(35.00 \%)$ & $4(40.00 \%)$ & $5(35.70 \%)$ & $6(37.50 \%)$ \\
\hline Fire/explotion & $20(38.50 \%)$ & $15(39.50 \%)$ & $8(44.40 \%)$ & $4(33.30 \%)$ & $7(35.00 \%)$ & $5(50.00 \%)$ & $5(35.70 \%)$ & $6(37.50 \%)$ \\
\hline $\begin{array}{l}\text { Transportation } \\
\text { accident }\end{array}$ & $37(71.20 \%)$ & $28(73.70 \%)$ & $12(66.70 \%)$ & $8(66.70 \%)$ & $15(75.00 \%)$ & $7(70.00 \%)$ & $10(71.40 \%)$ & $13(81.30 \%)$ \\
\hline $\begin{array}{l}\text { Serious accident at } \\
\text { work/home/rec }\end{array}$ & $23(44.20 \%)$ & $22(57.90 \%)$ & $9(50.00 \%)$ & $6(50.00 \%)$ & $8(40.00 \%)$ & $6(60.00 \%)$ & $6(42.90 \%)$ & $10(62.50 \%)$ \\
\hline $\begin{array}{l}\text { Exposure to toxic } \\
\text { substances }\end{array}$ & $13(25.00 \%)$ & $8(21.10 \%)$ & $7(38.90 \%)$ & $2(16.70 \%)$ & $4(20.00 \%)$ & $3(30.00 \%)$ & $2(14.30 \%)$ & $2(12.50 \%)$ \\
\hline Physical assault & $35(67.30 \%)$ & $21(55.30 \%)$ & $15(83.30 \%)$ & $7(58.30 \%)$ & $10(50.00 \%)$ & $4(40.00 \%)$ & $10(71.40 \%)$ & $10(62.50 \%)$ \\
\hline Assault with weapon & $14(26.90 \%)$ & $14(36.80 \%)$ & $4(22.20 \%)$ & $4(33.30 \%)$ & $5(25.00 \%)$ & $2(20.00 \%)$ & $5(35.70 \%)$ & $8(50.00 \%)$ \\
\hline Sexual assault & $24(46.20 \%)$ & $23(60.50 \%)$ & $9(50.00 \%)$ & $7(58.30 \%)$ & $8(40.00 \%)$ & $5(50.00 \%)$ & $7(50.00 \%)$ & $11(68.80 \%)$ \\
\hline $\begin{array}{l}\text { Other unwanted } \\
\text { sexual experience }\end{array}$ & $33(63.50 \%)$ & $25(65.80 \%)$ & $15(83.30 \%)$ & $8(66.70 \%)$ & $10(50.00 \%)$ & $6(60.00 \%)$ & $8(57.10 \%)$ & $11(68.80 \%)$ \\
\hline $\begin{array}{l}\text { Combat/exposure to } \\
\text { war }\end{array}$ & $1(1.90 \%)$ & $4(10.50 \%)$ & $0(00.00 \%)$ & $1(8.30 \%)$ & $0(0.00 \%)$ & $2(20.00 \%)$ & $1(7.10 \%)$ & $1(6.30 \%)$ \\
\hline
\end{tabular}




\begin{tabular}{|c|c|c|c|c|c|c|c|c|}
\hline Captivity & $2(3.80 \%)$ & $5(13.20 \%)$ & $1(5.60 \%)$ & $2(16.70 \%)$ & $0(0.00 \%)$ & $1(10.00 \%)$ & $1(7.10 \%)$ & $2(12.50 \%)$ \\
\hline $\begin{array}{l}\text { Life-threatening } \\
\text { illness/injury }\end{array}$ & $33(63.50 \%)$ & $26(68.40 \%)$ & $11(61.10 \%)$ & $8(66.70 \%)$ & $11(55.00 \%)$ & $5(50.00 \%)$ & $11(78.60 \%)$ & $13(81.30 \%)$ \\
\hline $\begin{array}{l}\text { Severe human } \\
\text { suffering }\end{array}$ & $20(34.60 \%)$ & $20(52.60 \%)$ & $7(38.90 \%)$ & $7(58.30 \%)$ & $7(35.00 \%)$ & $6(60.00 \%)$ & $6(42.90 \%)$ & $9(56.30 \%)$ \\
\hline Sudden violent death & $24(46.20 \%)$ & $16(42.10 \%)$ & $5(38.90 \%)$ & $5(41.70 \%)$ & $10(50.00 \%)$ & $4(40.00 \%)$ & $7(50.00 \%)$ & $7(43.80 \%)$ \\
\hline $\begin{array}{l}\text { Sudden accidental } \\
\text { death }\end{array}$ & $21(40.40 \%)$ & $21(55.30 \%)$ & $10(55.60 \%)$ & $6(50.00 \%)$ & $6(30.00 \%)$ & $5(50.00 \%)$ & $5(35.70 \%)$ & $10(62.50 \%)$ \\
\hline $\begin{array}{l}\text { Serious } \\
\text { injury/harm/death you } \\
\text { caused }\end{array}$ & $3(5.80 \%)$ & $3(7.90 \%)$ & $0(0.00 \%)$ & $0(0.00 \%)$ & $1(5.00 \%)$ & $1(10.00 \%)$ & $2(14.30 \%)$ & $2(12.50 \%)$ \\
\hline
\end{tabular}




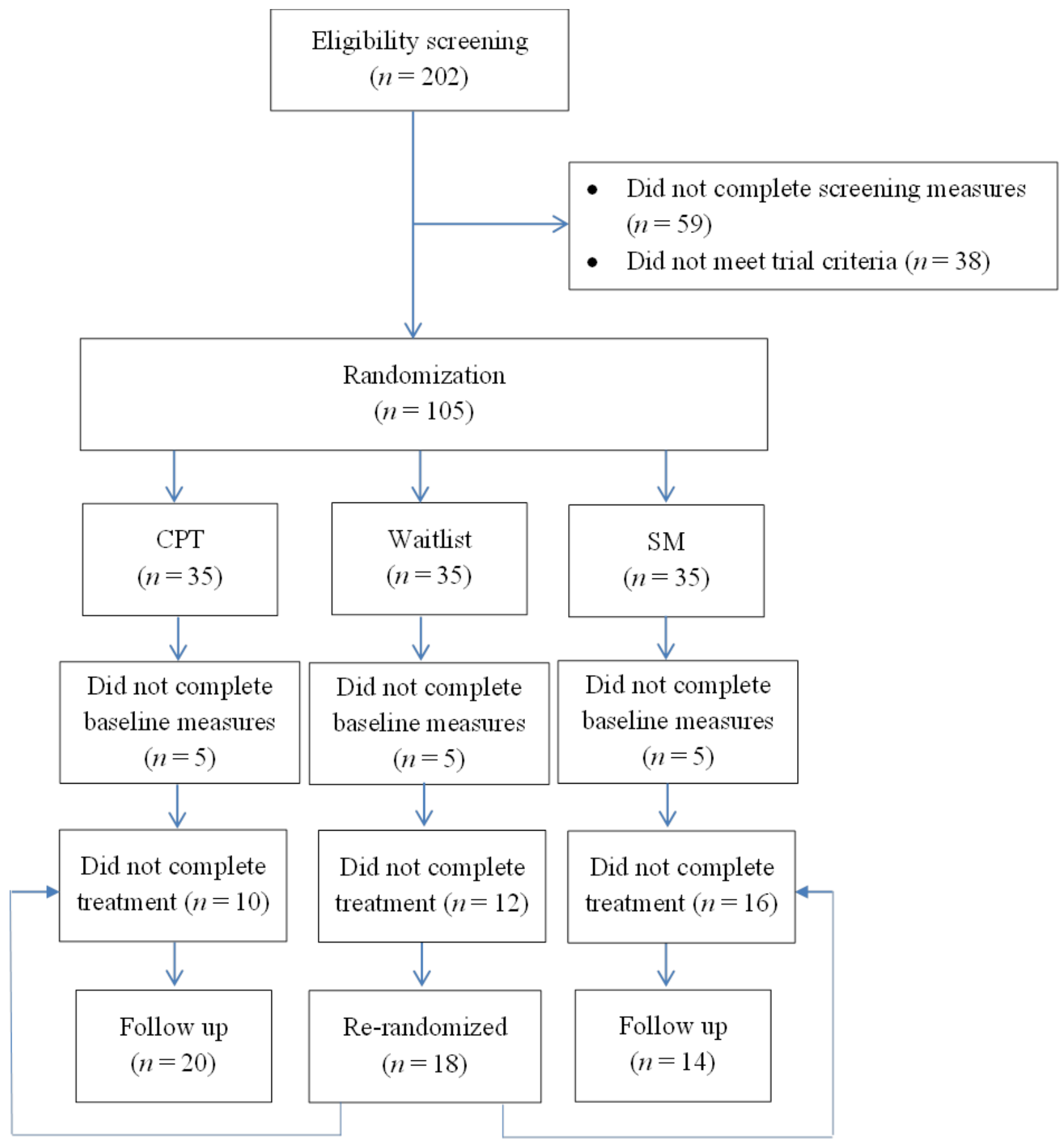

Figure 2. Participant flow. Data from a total of 52 participants was included in the analyses. Sample sizes for SM and CPT include the 18 participants who completed the waitlist and were re-randomized. $\mathrm{CPT}=$ Cognitive Processing Therapy; $\mathrm{SM}=$ Stress Management 
Figure 3. Session-by-session changes in SIPS, DASS-21, and DAR-5 scores in CPT

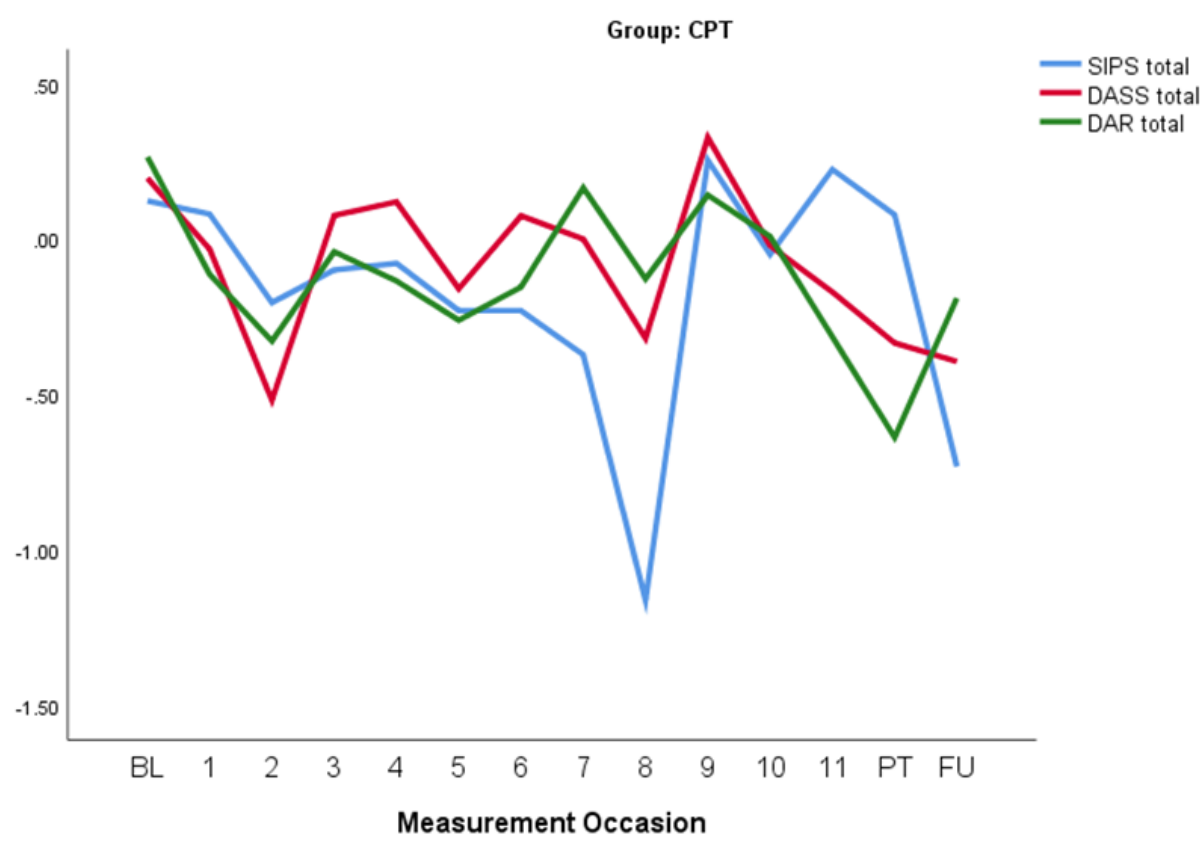

Figure 4. Session-by-session changes in SIPS, DASS-21, and DAR-5 scores in SM

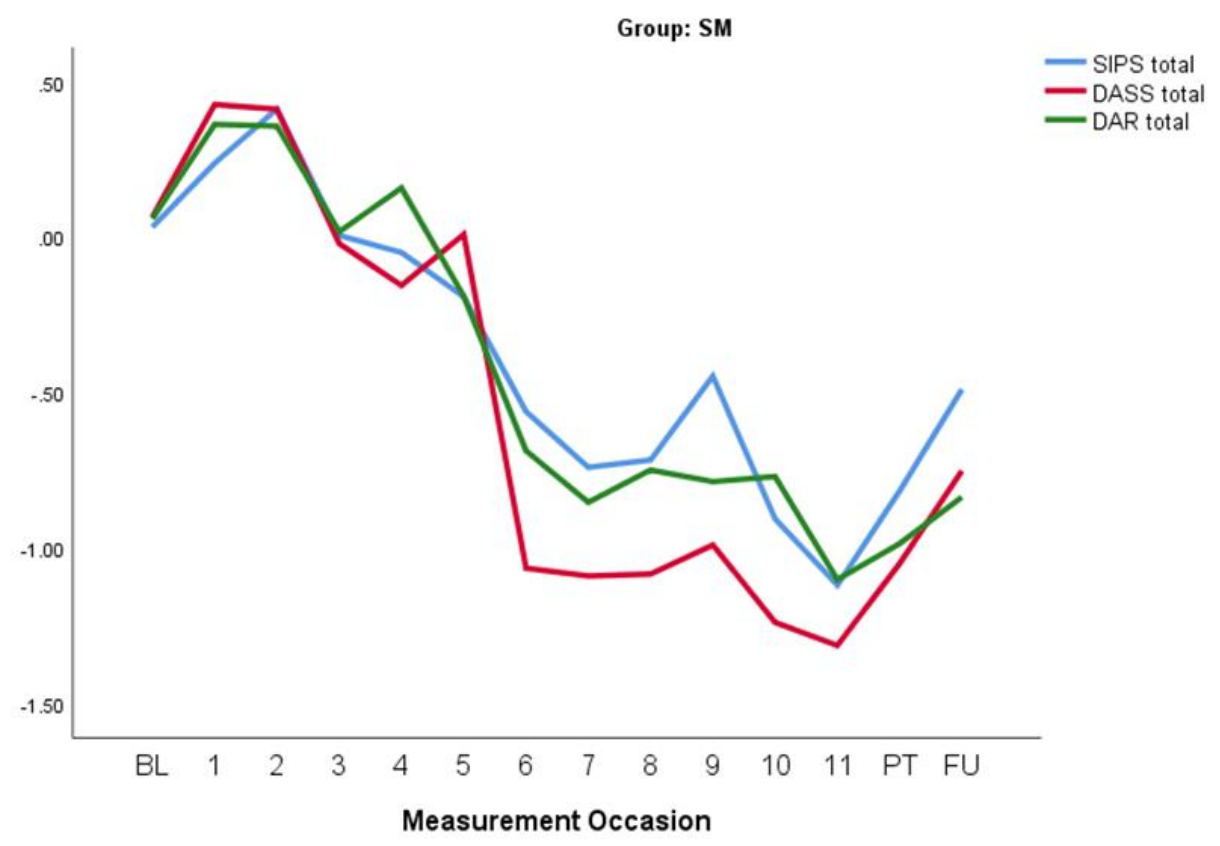




\subsection{Primary Outcomes}

Hypothesis one and two were tested using HLM. A forward step-wise selection was utilized where model fit was compared with increased parameter complexity. For all HLM models, the best fitting models did not include the random effect of time.

\subsubsection{Maladaptive trauma appraisals: PTCI}

Mean total PTCI scores increased over time in both treatment groups compared to the waitlist (see Table 5). CPT was associated with a Cohen's $d$ of 0.69 (95\% CI [.20, 1.19]) while SM was associated with a Cohen's $d$ of 0.84 (95\% CI [.19, 1.48]). However, number of lifetime bullying victimization experiences was a significant predictor in CPT and mean total PTCI scores decreased over time for participants who had a single lifetime exposure to bullying victimization compared to the waitlist and CPT participants with more than one bullying victimization experience. This interaction effect was associated with a Cohen's $d$ of -0.86 (95\% CI [-1.51, -.22]). Number of lifetime bullying victimization experiences was not a significant predictor in the SM group. Number of treatment sessions completed and time of bullying victimization experiences did not produce significant effects or improve fit in any of the models tested.

Mean total scores on the PTCI Negative Self-Beliefs subscale increased over time in both treatment groups compared to the waitlist. CPT was associated with a Cohen's $d$ of $0.71(95 \%$ CI $[.29,1.13])$, while SM was associated with a Cohen's $d$ of 0.54 (95\% CI $[-.01,1.07])$. However, number of bullying victimization experiences was a significant predictor over time in CPT, and mean total PTCI Negative Self-Beliefs scores decreased over time for participants in CPT who had a single lifetime bullying victimization experience compared to the waitlist 
and CPT participants with more than one bullying victimization experience. This effect was associated with a Cohen's $d$ of $-0.84(95 \%$ CI [-1.39, -.28]). Number of lifetime bullying victimization experiences was not a significant predictor in the SM group. Number of treatment sessions completed and time of bullying victimization experiences did not produce significant effects or improved fit in any of the models tested (see Table $6)$.

Mean total scores on the PTCI Negative Other-Beliefs subscale increased over time in SM compared to the waitlist. SM was associated with a Cohen's $d$ of 0.98 (95\% CI $[4.35,1.53])$. No significant differences were found in CPT compared to the waitlist over time (see Table 7). However, number of lifetime bullying victimization experiences was a significant predictor over time in both CPT and SM, and mean total PTCI Negative Other-Beliefs scores decreased significantly for participants that had a single lifetime exposure to bullying victimization experience compared to the waitlist, and participants with more than one bullying victimization experience. This interaction was associated with a Cohen's $d$ of $-0.60(95 \%$ CI $[-1.19,-.01])$ in CPT, and a Cohen's $d$ of $-0.73(95 \%$ CI $[-1.38,-.07])$ in SM. Number of treatment sessions completed and time of bullying victimization experiences did not produce significant effects or improve fit in any of the models tested.

Mean total scores on the PTCI Self-Blame subscale increased over time in SM compared to the waitlist. SM was associated with a Cohen's $d$ of 0.68 (95\% CI [.13, 1.21]). No significant differences were found in PTCI Self-Blame scores in CPT compared to the waitlist (see Table 8). Number of treatment sessions completed, time of 
bullying victimization experiences, and number of lifetime bullying victimization experiences did not produce significant effects or improve fit in any of the models tested. 
Table 5. Multilevel Model of PTCI total scores over time

\begin{tabular}{|c|c|c|c|c|c|}
\hline & Estimate & $S E$ & $d f$ & $t$ & $p$ \\
\hline \multicolumn{6}{|l|}{ Fixed effects } \\
\hline (Intercept) & $139.48[108.25,170.72]$ & 15.57 & 52.87 & 8.96 & $.001 * * *$ \\
\hline Group (CPT) & $-52.89[-97.56,-8.22]$ & 21.65 & 24.27 & -2.44 & $.022 *$ \\
\hline Group (SM) & $-77.53[-140.52,-14.55]$ & 31.10 & 37.71 & -2.49 & $.017 *$ \\
\hline Time & $-6.21[-20.01,7.58]$ & 6.62 & 20.33 & -.94 & .359 \\
\hline NBV & $-26.98[-72.38,18.41]$ & 22.64 & 53.62 & -1.19 & .238 \\
\hline Group $x$ time $(\mathrm{CPT})$ & $26.18[4.03,48.33]$ & 10.61 & 19.78 & 2.47 & $.023^{*}$ \\
\hline Group x time (SM) & $36.99[8.08,65.89]$ & 13.75 & 17.91 & 2.69 & $.015^{*}$ \\
\hline $\begin{array}{l}\text { Group } \times \text { time } x \\
\text { NBV }(\mathrm{CPT})\end{array}$ & $-30.31[-58.90,-1.71]$ & 13.83 & 23.04 & -2.19 & $.039 *$ \\
\hline $\begin{array}{l}\text { Group } \times \text { time } x \\
\text { NBV }(\text { SM })\end{array}$ & $-23.82[-58.17,10.52]$ & 16.34 & 17.82 & -1.46 & .162 \\
\hline \multicolumn{6}{|l|}{ Random effects } \\
\hline Intercept & $1034.66[648.33,1651.22]$ & & & & $.001 * * *$ \\
\hline Baseline & $417.40[107.78,1616.54]$ & & & & .148 \\
\hline Post-treatment & $185.33[44.06,779.53]$ & & & & .172 \\
\hline Follow-up & $233.42[65.21,835.57]$ & & & & .124 \\
\hline
\end{tabular}

Note . $\mathrm{CPT}=$ Cognitive Processing Therapy, $\mathrm{SM}=$ Stress Management, NBV= Number of bullying victimization experiences. Waitlist was used as a comparison group. ${ }^{*} p<.05 .{ }^{*} p p .01$. $* * * p<.001$.

Table 6. Multilevel Model of PTCI Negative Self-Beliefs Scores over time

\begin{tabular}{|c|c|c|c|c|c|}
\hline & Estimate & $S E$ & $d f$ & $t$ & $p$ \\
\hline $\begin{array}{l}\text { Fixed effects } \\
\text { (Intercept) }\end{array}$ & $83.19[65.07,101.30]$ & 9.03 & 52.55 & 9.21 & $.001 * * *$ \\
\hline Group (CPT) & $-39.51[-63.10,-15.92]$ & 11.71 & 44.26 & -3.38 & $.002 * *$ \\
\hline Group (SM) & $-31.10[-65.66,3.60]$ & 17.14 & 53.30 & -1.82 & .077 \\
\hline Time & $-4.73[-11.62,2.16]$ & 3.24 & 15.66 & -1.46 & .165 \\
\hline NBV & $-25.08[-52.22,2.05]$ & 13.53 & 53.28 & -1.85 & .069 \\
\hline Group x time (CPT) & $18.73[7.63,29.84]$ & 5.41 & 26.52 & 3.46 & $.002 * *$ \\
\hline Group x time (SM) & $14.20[.16,28.23]$ & 6.57 & 14.54 & 2.69 & $.048 *$ \\
\hline $\begin{array}{l}\text { Group } \times \text { time } x \text { NBV } \\
(\mathrm{CPT})\end{array}$ & $-22.15[-36.79,-7.50]$ & 7.11 & 25.13 & -3.11 & $.005 * *$ \\
\hline $\begin{array}{l}\text { Group } \times \text { time } \times \text { NBV } \\
(\mathrm{SM})\end{array}$ & $-11.77[-28.45,4.92]$ & 7.83 & 15.10 & -1.50 & .154 \\
\hline \multicolumn{6}{|l|}{ Random effects } \\
\hline Intercept & $503.81[326.55,777.31]$ & & & & $.001 * * *$ \\
\hline Baseline & $39.86[4.34,365.76]$ & & & & .377 \\
\hline Post-treatment & $108.55[38.83,303.49]$ & & & & .057 \\
\hline Follow-up & $112.59[38.82,777.31]$ & & & & .066 \\
\hline
\end{tabular}

Note. CPT $=$ Cognitive Processing Therapy, $\mathrm{SM}=$ Stress Management, NBV= Number of Bullying Victimization experiences. Waitlist was used as a comparison group. ${ }^{*} p<.05$. ${ }^{*} p<<$ $.01 . * * * p<.001$. 
Table 7. Multilevel Model of PTCI Negative Other-Beliefs scores over time

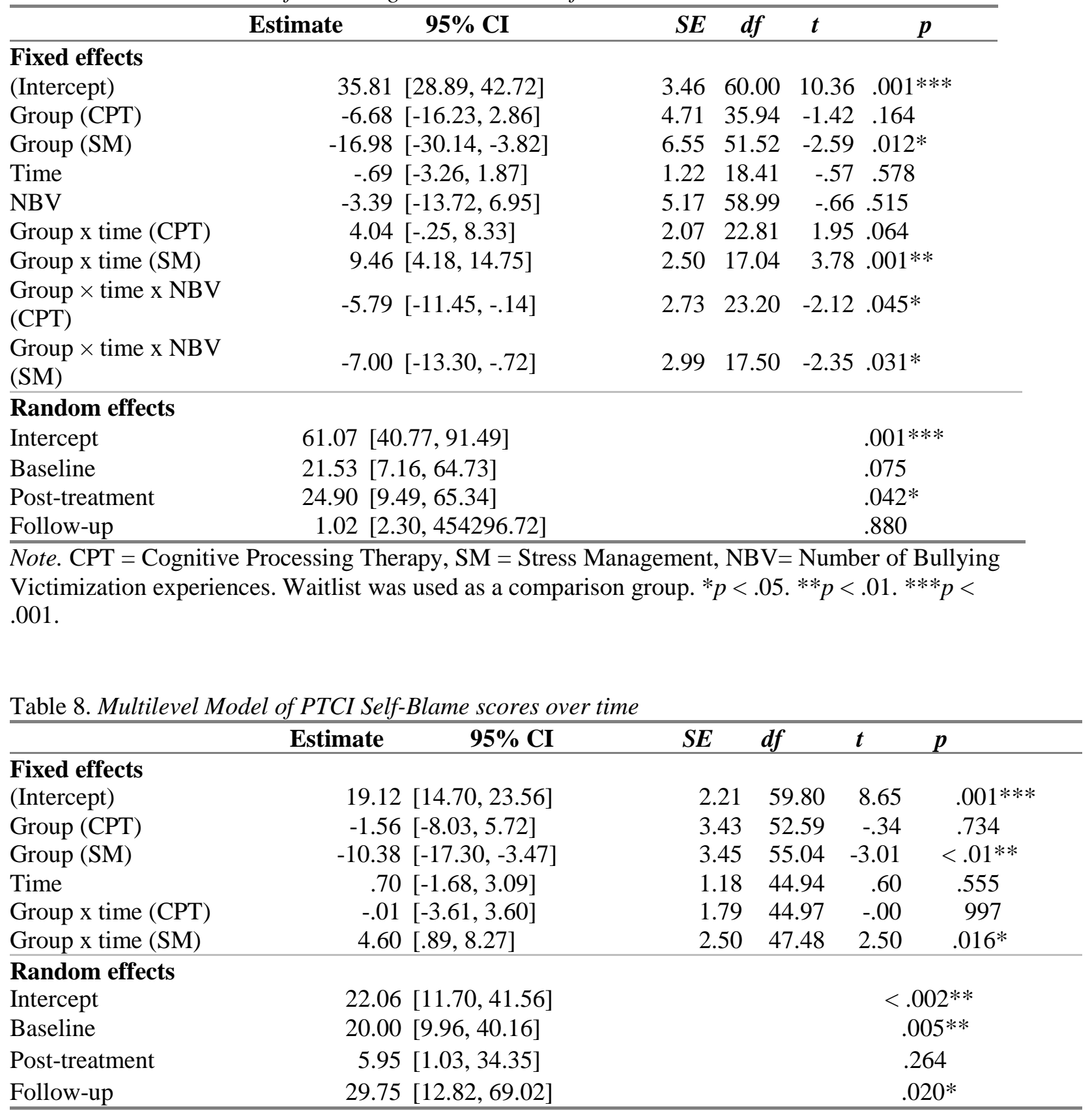

Note. CPT $=$ Cognitive Processing Therapy, $\mathrm{SM}=$ Stress Management. Waitlist was used as a comparison group. $* p<.05 . * * p<.01 . * * * p<.001$. 
Table 9. Multilevel Model of PCL-5 scores over time

\begin{tabular}{|c|c|c|c|c|c|}
\hline & Estimate & $S E$ & $d f$ & $t$ & $p$ \\
\hline \multicolumn{6}{|l|}{ Fixed effects } \\
\hline (Intercept) & $37.33[24.86,49.97]$ & 5.95 & 16.55 & 6.29 & $.001 * * *$ \\
\hline Group (CPT) & $-15.53[-40.11,9.05]$ & 12.30 & 61.84 & -1.26 & .211 \\
\hline Group (SM) & $-14.50[-38.30,9.30]$ & 11.91 & 61.84 & -1.22 & .228 \\
\hline Post-treatment & $1.78 \quad[-12.51,16.07]$ & 6.47 & 10.63 & .28 & .788 \\
\hline Follow-up & $6.36[-7.21,14.77]$ & 7.21 & 14.77 & .88 & .392 \\
\hline TBV & $7.27[-13.27,27.80]$ & 9.71 & 16.55 & -.75 & .465 \\
\hline $\begin{array}{l}\text { Group x post } \\
\text { (CPT) }\end{array}$ & $-15.70[-27.37,-4.03]$ & 9.44 & 23.57 & -2.62 & $.015^{*}$ \\
\hline Group x fu (CPT) & $-25.13[-46.23,-4.03]$ & 10.04 & 17.94 & -2.50 & $.022 *$ \\
\hline $\begin{array}{l}\text { Group x post } \\
\text { (SM) }\end{array}$ & $-14.82[-33.73,4.03]$ & 12.26 & 25.67 & -2.05 & .051 \\
\hline Group x fu (SM) & $-8.00 \quad[-16.83, .85]$ & 4.28 & 23.84 & $\begin{array}{r}- \\
1.87\end{array}$ & .074 \\
\hline TBV $\mathrm{x}$ post & $-9.00[-31.11,13.09]$ & 10.45 & 16.51 & -.86 & .401 \\
\hline TBV $x$ fu & $-19.42[-38.77,-.075]$ & 9.11 & 15.60 & -2.13 & $.049 *$ \\
\hline \multicolumn{6}{|l|}{ Random effects } \\
\hline Intercept & $188.67[88.84,419.56]$ & \multicolumn{4}{|c|}{$<.014 * *$} \\
\hline Baseline & $165.09[75.97,358.76]$ & \multicolumn{4}{|c|}{$.012 * *$} \\
\hline Post-treatment & $89.35[9.57,834.29]$ & \multicolumn{4}{|c|}{.380} \\
\hline Follow-up & $25.19[.10,6171.33]$ & \multicolumn{4}{|c|}{.722} \\
\hline
\end{tabular}

Note . $\mathrm{CPT}=$ Cognitive Processing Therapy, $\mathrm{SM}=$ Stress Management, $\mathrm{TBV}=$ Time of Bullying

Victimization experiences. Waitlist was used as a comparison groups. $* p<.05$. $* * p<.01 . * * * p<$ .001 .

\subsubsection{Symptoms of PTSD: PCL-5}

Pairwise comparisons were made between measurement occasions in models for

PCL-5, as time was a significant covariate. Mean total scores reduced significantly on the PCL-5 from baseline to post-treatment, and baseline to follow-up in CPT compared to the waitlist (see Table 9). CPT was associated with a Cohen's $d$ of -0.86 (95\% CI [-1.98, .537]). No significant differences in PCL-5 mean scores were found in SM over time compared to the waitlist. Number of treatment sessions completed and number of lifetime bullying victimization experiences did not produce significant effects or improve fit in any of the models tested. Time of bullying victimization was a significant predictor for PCL-5 scores over time, across all study groups, with remote bullying experiences 
associated with a significantly greater reduction in PCL-5 scores over time, compared to recent bullying victimization experience. No cross-level interaction effects of time of bullying victimization with group were, however, significant.

\subsubsection{Social anxiety symptoms: SIPS}

No significant differences were found between mean total SIPS scores in CPT or SM compared to the waitlist over time (Table 10). Number of treatment sessions completed, number of lifetime bullying victimization experiences, and time of bullying victimization experiences did not produce significant effects or improve model fit in any of the models tested. Time was a significant covariate for total SIPS scores and all models were, thus, rerun with the time variable as a factor. However, rerunning the models with time as a factor did not produce significant associations between any of the predictor variables and SIPS total scores.

Mean total SIPS SIA scores increased over time in CPT compared to the waitlist (Table 11). CPT was associated with a Cohen's $d$ of 0.29 (95\% CI $[.08, .50])$. No significant differences were found in total mean SIPS SIA scores over time in SM compared to the waitlist. Number of treatment sessions completed, number of lifetime bullying victimization experiences, and time of bullying victimization did not produce significant effects or improve fit in any of the models tested. Pairwise comparisons were made between measurement occasions in the models for the SIPS FOE subscale as time was a significant covariate. Mean total SIPS FOE scores increased from baseline to follow-up in SM compared to the waitlist. SM was associated with a Cohen's $d$ of -0.50 $(95 \%$ CI $[.00, .99])$. No significant differences in mean total SIPS FOE scores were found in SM from baseline to post-treatment compared to the waitlist, or between CPT 
and the waitlist from baseline to post-treatment and baseline to follow-up (see Table 12). Number of treatment sessions completed, number of lifetime bullying victimization experiences, and time of bullying victimization experiences did not produce significant effects or improve fit in any of the models tested. Mean total scores on the SIPS SAA subscale increased over time in CPT, compared to the waitlist (see Table 13). CPT was associated with a Cohen's $d$ of 0.30 (95\% CI [-1.47, .44]. No significant differences in mean total SIPS SAA scores were found for SM compared to the waitlist. Number of treatment sessions completed, time of bullying victimization experiences, and number of bullying victimization did not produce significant effects or improve fit in any of the models tested. 
Table 10. Multilevel Model of SIPS total scores over time

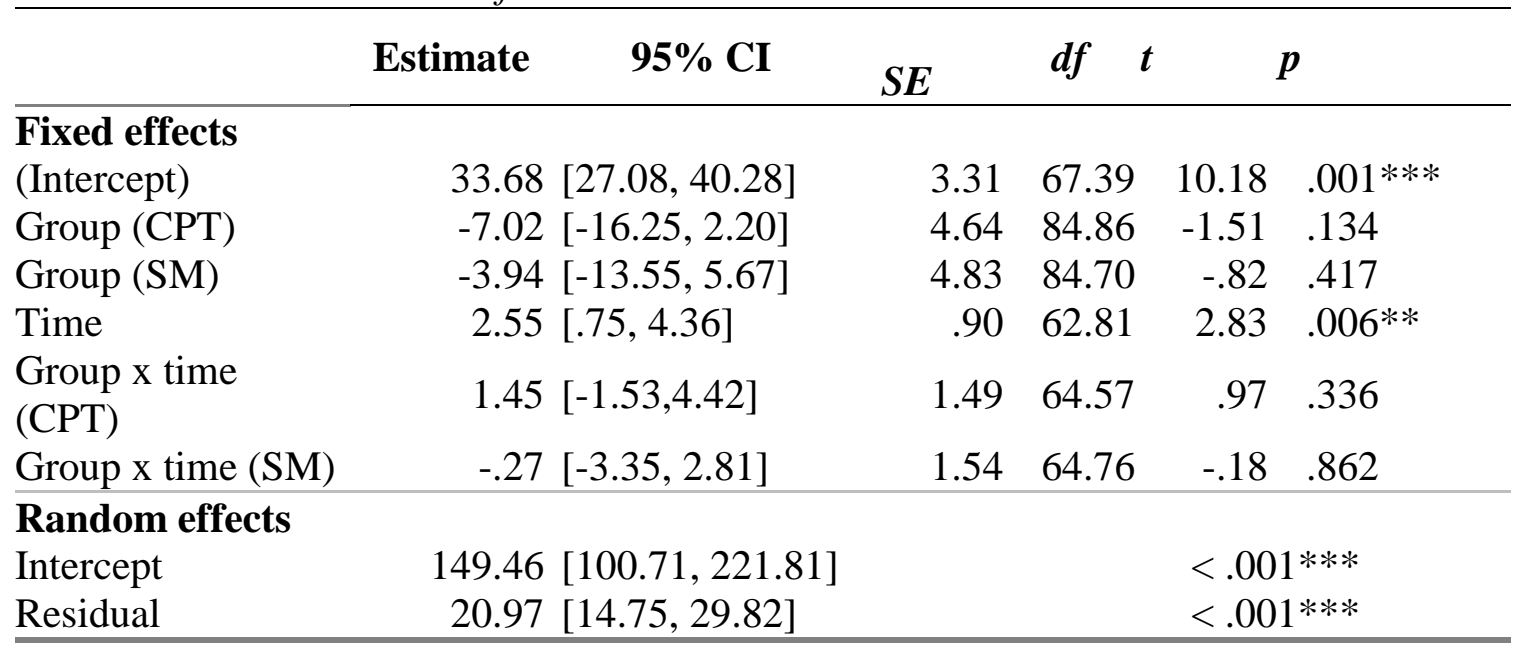

Note . CPT $=$ Cognitive Processing Therapy, $\mathrm{SM}=$ Stress Management. Waitlist was used as comparison group. $* p<.05 . * * p<.01 . * * * p<.001$.

Table 11. Multilevel Model of SIPS SIA scores over time

\begin{tabular}{|c|c|c|c|c|c|c|}
\hline & Estimate & $95 \% \mathrm{CI}$ & $S E$ & $d f$ & $t$ & $p$ \\
\hline \multicolumn{7}{|l|}{ Fixed effects } \\
\hline (Intercept) & 14.78 & $\begin{array}{l}{[12.07,} \\
17.48]\end{array}$ & 1.34 & 45.77 & 11.01 & $.001 * * *$ \\
\hline Group (CPT) & -3.18 & {$[-6.95, .60]$} & 1.88 & 55.44 & -1.69 & .098 \\
\hline Group (SM) & -1.28 & {$[-5.21,2.66]$} & 1.96 & 54.67 & -.65 & .518 \\
\hline Time & .45 & {$[-.69, .78]$} & .36 & 29.21 & .12 & .903 \\
\hline Group x time (CPT) & 1.74 & {$[.49,2.99]$} & .62 & 34.00 & 2.83 & $.008 * *$ \\
\hline Group x time (SM) & 1.05 & {$[-.23,2.33]$} & .63 & 32.00 & 1.67 & .104 \\
\hline \multicolumn{7}{|l|}{ Random effects } \\
\hline Intercept & 27.16 & {$[17.87,41.26$} & & & & $\leq .001 * * *$ \\
\hline Baseline & 10.83 & {$[6.28,18.67]$} & & & & $\leq .001 * * *$ \\
\hline Post-treatment & 2.88 & {$[1.29,6.46]$} & & & & $.016^{*}$ \\
\hline Follow-up & .47 & {$[.01,33.31]$} & & & & .645 \\
\hline
\end{tabular}

Note. CPT $=$ Cognitive Processing Therapy, $\mathrm{SM}=$ Stress Management. Waitlist was used as comparison group. ${ }^{*} p<.05 . * * p<.01 . * * * p<.001$. 
Table 12. Multilevel Model of SIPS FOE scores over time

\section{$\begin{array}{lllllll}\text { Estimate } & 95 \% & \mathrm{CI} & S E & d f & t & p\end{array}$}

\section{Fixed effects}

(Intercept)

Group (CPT)

Group (SM)

Post tx

$\mathrm{Fu}$

Group x post-tx (CPT)

Group x fu (CPT)

Group x post-tx (SM)

Group x fu (SM)

Random effects

Intercept

$28.65[19.10,42.99]$

Baseline

Post-treatment

Follow-up $\begin{array}{llll}1.55 & 74.08 & 9.49 & .001 * * *\end{array}$

$\begin{array}{lllll}2.07 & 74.08 & -.98 & .332\end{array}$

$\begin{array}{llll}2.15 & 74.08 & -1.67 & .100\end{array}$

$\begin{array}{llll}.95 & 45.80 & -1.70 & .009 * *\end{array}$

$-1.63[-3.48, .22]$

$.93 \quad 36.56-2.76$

.096

$07[-2.63$,

2.77]

$1.35 \quad 49.29$

.05

.958

$.66 \frac{[-2.16,}{3.48]}$

$\begin{array}{lll}1.40 & 46.72 \quad .47\end{array}$

.640

$1.79[-1.09$,

$2.95[-1.09$, 4.66]
$1.43 \quad 50.10 \quad 1.25$

.217

$1.46 \quad 45.46 \quad 2.02 \quad .049 *$

Note. CPT $=$ Cognitive Processing Therapy, $\mathrm{SM}=$ Stress Management, Post-tx $=$ Post Treatment, $\mathrm{Fu}=$ Follow-up. Waitlist was used as a comparison group. ${ }^{*} p<.05 . * * p<.01 . * * * p<.001$.

Table 13. Multilevel Model of SIPS SAA scores over time

\begin{tabular}{lcrrrrr}
\hline & Estimate & $\mathbf{9 5 \%} \mathbf{C I}$ & $\boldsymbol{S E}$ & $\boldsymbol{d} \boldsymbol{f}$ & $\boldsymbol{t}$ & $\boldsymbol{p}$ \\
\hline $\begin{array}{l}\text { Fixed effects } \\
\text { (Intercept) }\end{array}$ & $7.57[6.18,8.96]$ & 69. & 53.34 & 10.93 & $.001^{* * *}$ \\
Group (CPT) & $-3.08[-5.06,-$ & .99 & 66.83 & -3.12 & $.003^{* *}$ \\
Group (SM) & $-1.68[-3.74, .39]$ & 1.03 & 65.95 & -1.63 & .108 \\
Time & $.00[-.44, .44]$ & .22 & 27.62 & .00 & .997 \\
Group x time (CPT) & $.84[.09,1.58]$ & .37 & 32.67 & 2.28 & $.029 *$ \\
Group x time (SM) & $.45[-.31,1.21]$ & .37 & 30.86 & 1.21 & .237 \\
Random effects & & & & & \\
Intercept & $7.00[4.63,10.58]$ & & & & $\leq .001^{* * *}$ \\
Baseline & $4.13[2.56,6.67]$ & & & & $\leq .001^{* * *}$ \\
Post-treatment & $.70[.26,1.94]$ & & & & $.053^{*}$ \\
Follow-up & $.27[.03,2.58]$ & & & & .383 \\
\hline
\end{tabular}

Note. $\mathrm{CPT}=$ Cognitive Processing Therapy, $\mathrm{SM}=$ Stress Management. Waitlist was used as a comparison group. $* p<.05 . * * p<.01 . * * * p<.001$. 


\subsubsection{Distress: DASS-21}

Mean total scores on the DASS-21 decreased significantly over time in SM compared to the waitlist (see Table 14). SM was associated with a Cohen's $d$ of -1.06 (95\% CI $[-1.74,-.39])$. No significant differences were found over time between CPT and the waitlist. However, when number of treatment sessions completed was added into the models as a predictor, mean total DASS-21 scores decreased for participants in CPT that completed more sessions compared to CPT participants that completed fewer sessions, and compared to the waitlist. Participants in SM that completed more treatment sessions had also significantly lower total mean scores over time, compared to the waitlist and SM participants that completed fewer sessions. This interaction effect in CPT was associated with a Cohen's $d$ of -0.10 (95\% CI [-.17, -.02]), while in SM it was associated with a Cohen's $d$ of -0.03 (95\% CI [-.17, -.02]). Time of bullying victimization experiences and number of lifetime bullying victimization experiences did not produce significant effects or improved fit in any of the models tested.

Mean total scores on the DASS-21 Depression subscale decreased significantly over time in SM compared to the waitlist. SM was associated with a Cohen's $d$ of -0.02 (95\% CI $[-.33,30])$. No significant differences were found in CPT compared to the waitlist (see Table 15). Number of treatment sessions completed produced a significant main effect, but no interaction effects with treatment groups were found. Time of bullying victimization experiences and number of lifetime bullying victimization experiences did not produce significant effects or improved fit in any of the models tested. 
Mean total scores on the DASS-21 Anxiety subscale decreased significantly over time in the SM compared to the waitlist. SM was associated with a Cohen's $d=-1.10$ (95\% CI [-2.06, -.13]). No significant differences were found in CPT compared to the waitlist. However, number of treatment sessions completed was a significant predictor in CPT and mean total scores on the DASS-21 Anxiety subscale decreased for participants that completed more treatment sessions compared to participants that completed fewer CPT sessions and compared to the waitlist. This interaction was associated with a Cohen's $d=-0.14$ (95\% CI [-.25, -.03]). Time of bullying victimization experiences and number of lifetime bullying victimization experiences did not produce significant effects or improved fit in any of the models tested (see Table 16).

No significant group and time interaction effects were found for DASS-21 Stress scores (Table 17). Number of treatment sessions completed was a significant predictor that improved model fit but did not produce significant interaction effects with the treatment groups. Time of bullying victimization experiences and number of lifetime bullying victimization experiences did not produce significant effects or improved fit in any of the models tested.

\subsubsection{Anger: DAR-5}

No significant differences were found in mean total scores on the DAR-5 in CPT or SM compared to the waitlist over time (see Table 18). Time of bullying victimization experiences produced a significant main effect, but no interaction effects with treatment groups were found. Number of treatment sessions completed and number of lifetime bullying victimization experiences did not produce significant effects or improve fit in any of the models tested. 
Table 14. Multilevel Model of DASS-21 total scores over time

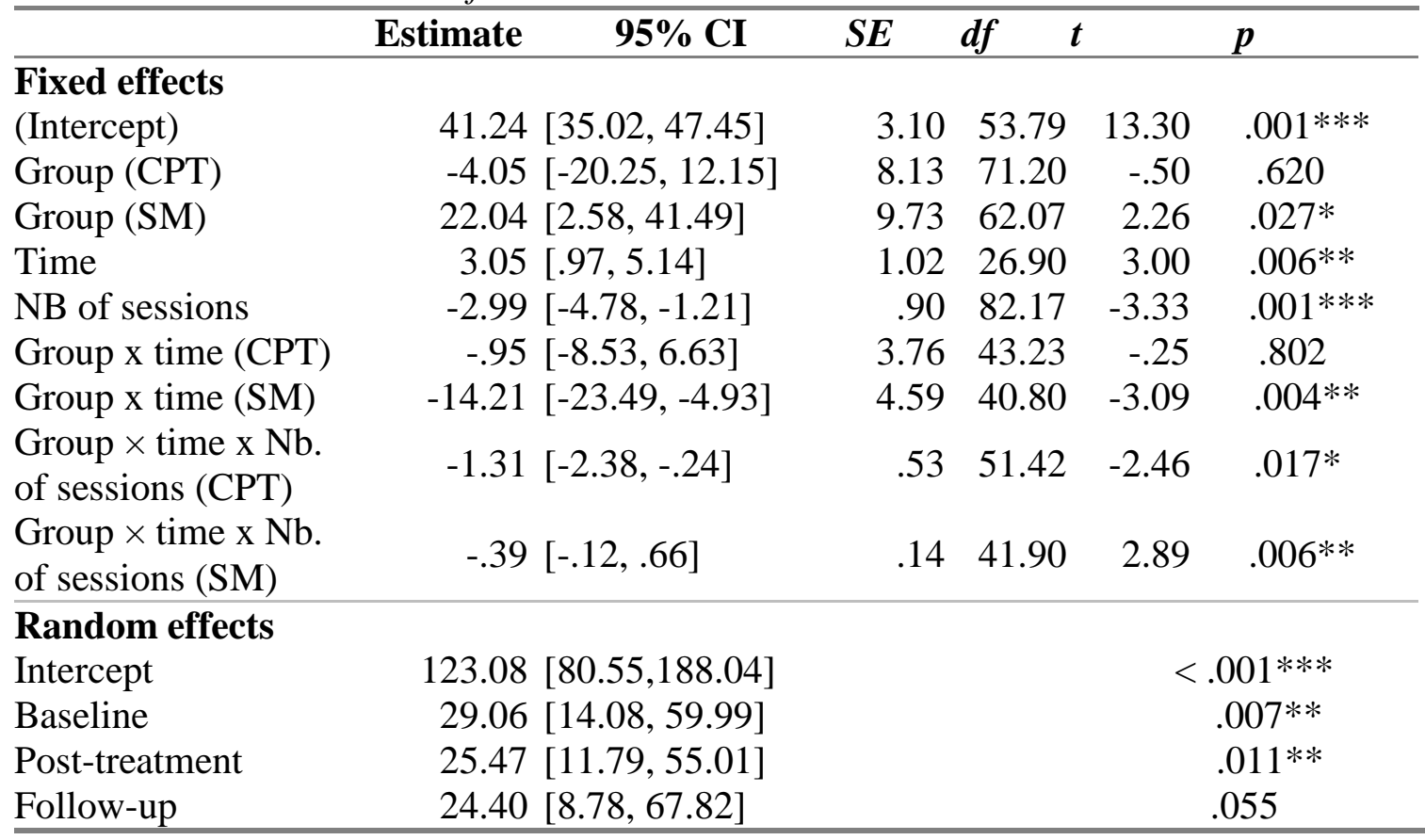

Note. CPT $=$ Cognitive Processing Therapy, $\mathrm{SM}=$ Stress Management, NB of sessions $=$

Number of treatment sessions completed. Waitlist was used as a comparison group. ${ }^{*} p<.05$.

$* * p<.01 . * * * p<.001$.

Table 15. Multilevel Model of DASS-21 Depression scores over time

\begin{tabular}{|c|c|c|c|c|c|}
\hline & Estimate & $S E$ & $d f$ & & $p$ \\
\hline $\begin{array}{l}\text { Fixed effects } \\
\text { (Intercept) }\end{array}$ & $14.31[11.99,16.62]$ & 1.15 & 43.12 & 12.47 & $.001 * * *$ \\
\hline Group (CPT) & $-2.87[-10.34,4.60]$ & 3.74 & 66.44 & -.77 & .445 \\
\hline Group (SM) & $7.17[-1.94,16.28]$ & 4.56 & 60.38 & 1.57 & .121 \\
\hline Time & $.96[.12,1.80]$ & .41 & 28.17 & 2.34 & $.027 *$ \\
\hline $\mathrm{Nb}$. of sessions & $-.87[-1.68,-.07]$ & .41 & 75.48 & -2.15 & $.035^{*}$ \\
\hline $\begin{array}{l}\text { Group x time } \\
\text { (CPT) }\end{array}$ & $.42[-3.31,4.15]$ & 1.86 & 52.07 & .23 & .823 \\
\hline $\begin{array}{l}\text { Group x time } \\
(\mathrm{SM})\end{array}$ & $-5.34[-9.92,-.75]$ & 2.28 & 50.15 & -2.34 & $.023 *$ \\
\hline
\end{tabular}

\section{Random effects}

$\begin{array}{lrl}\text { Intercept } & 17.85[11.53,27.62] & <.001^{* * *} \\ \text { Baseline } & 8.08[4.44,14.69] & <.001^{* * *} \\ \text { Post-treatment } & 6.42[3.24,12.69] & <.004 * * \\ \text { Follow-up } & 1.69[.26,11.13] & .299\end{array}$

Note . $\mathrm{CPT}=$ Cognitive Processing Therapy, $\mathrm{SM}=$ Stress Management. NB of sessions $=$ Number of treatment sessions completed.Waitlist was used as a comparison group. ${ }^{*} p<.05 .{ }^{*} p<.01$. $* * * p<.001$. 
Table 16. Multilevel Model of DASS-21 Anxiety scores over time

$\begin{array}{llllll}\text { Estimate } & 95 \% & \mathrm{CI} & S E & d f & \boldsymbol{t}\end{array}$

Fixed effects

(Intercept)

Group (CPT)

Group (SM)

Time

NB of sessions

Group x time (CPT)

Group $x$ time $(\mathrm{SM})$

Group $\times$ time $x$ NB of sessions (CPT)

Random effects

Intercept

Baseline

Post-treatment

Follow-up

$\begin{aligned} 11.63 & {[9.80,13.46] } \\ -4.16 & {[-10.75,2.43] } \\ 8.59 & {[.50,16.67] } \\ .87 & {[.06,1.68] } \\ -1.02 & {[-1.73,-.31] } \\ 1.47 & {[-1.95,4.90] } \\ -4.78 & {[-8.97,-.59] } \\ -.62 & {[-1.10,-.15] }\end{aligned}$

$10.55[6.36,17.50]$

$7.91[4.40,14.21]$

$5.21[2.63,10.33]$

$1.35[.19,9.64]$

$\begin{array}{rrrl}.91 & 42.69 & 12.83 & .001 * * * \\ 3.30 & 60.17 & -1.26 & .212 \\ 4.04 & 56.52 & 2.13 & .038^{*} \\ .39 & 20.63 & 2.24 & .036^{*} \\ .36 & 66.01 & -2.87 & .006^{* *} \\ 1.72 & 66.51 & .86 & .394 \\ 2.10 & 63.54 & -2.28 & .026 * \\ .24 & 75.52 & -2.63 & .016^{* *}\end{array}$

$\begin{array}{llll}.91 & 42.69 & 12.83 & .001 * * *\end{array}$

$\begin{array}{llll}3.30 & 60.17 & -1.26 & .212\end{array}$

$\begin{array}{llll}.04 & 56.52 & 2.13 & .038 *\end{array}$

$\begin{array}{llll}.39 & 20.63 & 2.24 & .036^{*}\end{array}$

$\begin{array}{llll}.36 & 66.01 & -2.87 & .006^{* *}\end{array}$

$\begin{array}{llll}1.72 & 66.51 & .86 & .394\end{array}$

$.24 \quad 75.52 \quad-2.63 \quad .010 * *$

Note. . CPT $=$ Cognitive Processing Therapy, $\mathrm{SM}=$ Stress Management, NB of sessions $=$

Number of treatment sessions completed. Waitlist was used as a comparison group. ${ }^{*} p<.05$.

$* * p<.01 . * * * p<.001$.

Table 17. Multilevel Model of DASS-21 Stress scores over time

\begin{tabular}{|c|c|c|c|c|c|c|}
\hline \multirow{2}{*}{\multicolumn{7}{|c|}{ Fixed effects }} \\
\hline & & & & & & \\
\hline (Intercept) & 15.22 & {$[12.54,17.90]$} & 1.34 & 73.23 & 11.33 & $.001 * * *$ \\
\hline Group (CPT) & 2.21 & {$[-4.44,8.86]$} & 3.33 & 68.59 & .66 & .509 \\
\hline Group (SM) & 5.42 & {$[-2.52,13.36]$} & 3.97 & 60.80 & 1.37 & .177 \\
\hline Time & 1.22 & {$[.21,2.22]$} & .49 & 35.91 & 2.46 & $.019 *$ \\
\hline NB of sessions & -1.01 & {$[-1.75,-.28]$} & .37 & 77.78 & -2.73 & $.008 * *$ \\
\hline $\begin{array}{l}\text { Group x time } \\
\text { (CPT) }\end{array}$ & -2.38 & {$[-5.48, .73]$} & 1.54 & 47.97 & -1.54 & .130 \\
\hline Group x time (SM) & -3.62 & {$[-7.38, .15]$} & 1.87 & 44.91 & -1.93 & .060 \\
\hline \multicolumn{7}{|l|}{ Random effects } \\
\hline Intercept & 20.01 & $\begin{array}{l}{[13.22,} \\
30.31]\end{array}$ & & & & $<.001 * * *$ \\
\hline Baseline & 4.27 & {$[1.86,9.78]$} & & & & $.018 *$ \\
\hline Post-treatment & 4.44 & {$[1.92,10.35]$} & & & & $.019 *$ \\
\hline Follow-up & 9.56 & {$[4.52,20.22]$} & & & & $.009 * *$ \\
\hline
\end{tabular}

Note. CPT $=$ Cognitive Processing Therapy, $\mathrm{SM}=$ Stress Management, NB of sessions $=$ Number of treatment sessions completed. Waitlist was used as a comparison group. ${ }^{*} p<.05$. ${ }^{* *} p<.01$. $* * * p<.001$. 
Table 18. Multilevel Model of DAR-5 scores over time

\begin{tabular}{|c|c|c|c|c|c|}
\hline & Estimate & $S E$ & $d f$ & & $p$ \\
\hline $\begin{array}{l}\text { Fixed effects } \\
\text { (Intercept) }\end{array}$ & $17.68[13.22,22.15]$ & 2.21 & 43.06 & 7.99 & $.001 * * *$ \\
\hline Group (CPT) & $-9.24[-15.12,-3.36]$ & 2.92 & 47.01 & -3.16 & $.003 * *$ \\
\hline Group (SM) & $-8.27[-16.94, .40]$ & 4.36 & 87.26 & -1.90 & .061 \\
\hline Time & $-.90[-3.22,1.42]$ & 1.14 & 33.19 & -.79 & .435 \\
\hline TBV & $-8.43[-13.20,-3.67]$ & 2.36 & 42.33 & -3.57 & $.001 * *$ \\
\hline Group $x$ time (CPT) & $1.79[-1.34,4.93]$ & 1.54 & 35.39 & 1.16 & .253 \\
\hline Group x time (SM) & $.45[-4.30,5.19]$ & 2.39 & 85.63 & .19 & .852 \\
\hline $\begin{array}{l}\text { Group } \times \text { TBV } \\
(\mathrm{CPT})\end{array}$ & $8.67[2.22,15.13]$ & 3.21 & 46.32 & 2.71 & $.010 * *$ \\
\hline Group x TBV (SM) & $6.52[-2.54,15.57]$ & 4.56 & 90.58 & 1.43 & .156 \\
\hline $\begin{array}{l}\text { Group } x \text { time } x \\
\text { TBV }(\mathrm{CPT})\end{array}$ & $-1.43[-4.84,1.99]$ & 1.68 & 35.23 & -.85 & .403 \\
\hline $\begin{array}{l}\text { Group } x \text { time } x \\
\text { TBV (SM) }\end{array}$ & $.73[-4.18,5.65]$ & 2.47 & 85.75 & .30 & .767 \\
\hline
\end{tabular}

\section{Random effects}

Intercept

$7.89[4.67,13.33]$

Baseline

Post-treatment

Follow-up
$9.54[5.70,15.99]$

$6.25[3.60,10.87]$

$.31[.00,141.45]$

$$
\begin{aligned}
& <.001 * * * \\
& <.001 * * * \\
& <.001 * * * \\
& .749
\end{aligned}
$$

Note. CPT $=$ Cognitive Processing Therapy, $\mathrm{SM}=$ Stress Management, TBV= Time of Bullying Victimization. Waitlist was used as a comparison groups. ${ }^{*} p<.05 . * * p<.01 . * * * p<.001$. 


\subsubsection{Distress tolerance: DTS}

No significant differences were found for in mean total DTS scores over time in CPT or SM compared to the waitlist (Table 19). Number of treatment sessions completed, number of lifetime bullying victimization experiences, and time of bullying victimization experiences did not produce significant effects or lead to improved fit in any of the models tested. Mean total scores on the DTS Absorption subscale decreased significantly in CPT and SM over time compared to the waitlist, indicating increased distress tolerance (see Table 20). CPT was associated with a Cohen's $d$ of -0.59 (95\% CI $[-1.08,-.11])$, while SM was associated with a Cohen's $d$ of -0.99 (95\% CI [-1.62, -.37]). Number of bullying victimization experiences was a significant predictor in SM, and participants with a single bullying victimization experience had significantly higher scores on the DTS Absorption scale over time compared to the waitlist and participants in SM that reported more than one bullying victimization experience. This interaction was associated with a Cohen's $d$ of 0.98 (95\% CI [.23, 1.73]). Number of treatment sessions completed and time of bullying victimization experiences did not produce significant effects or lead to improvement in fit in any of the models tested. No significant differences were found for any of the predictors tested (i.e., time, group, number of treatment sessions completed, number of bullying experiences, time of bullying experience) in total mean scores on the DTS Appraisal and DTS Regulation subscales (see Tables 21-22).

Mean total DTS Tolerance scores were significantly lower over time in SM compared to the waitlist (see Table 23). SM was associated with a Cohen's $d$ of -1.03 (95\% CI $[-1.56,-.50])$. No significant differences were found in CPT compared to the 
waitlist. Number of lifetime bullying victimization experiences was a significant predictor in SM, and mean total DTS Tolerance scores were significantly higher for participants in SM that had a single bullying victimization experience, compared to SM participants with more than one bullying victimization experience and the waitlist. This interaction was associated with a Cohen's $d$ of 0.84 (95\% CI [.21, 1.48]). Time of bullying victimization experiences and number of treatment sessions completed did not produce significant effects or improved fit in any of the models tested. 
Table 19. Multilevel Model of DTS Total scores over time

\begin{tabular}{|c|c|c|c|c|c|}
\hline & Estimate & $S E$ & $d f$ & & $p$ \\
\hline $\begin{array}{l}\text { Fixed effects } \\
\text { (Intercept) }\end{array}$ & $33.80[27.23,40.36]$ & 3.24 & 37.90 & 10.43 & $.001 * * *$ \\
\hline Group (CPT) & $6.08[-3.53,15.58]$ & 4.72 & 38.35 & 1.28 & .209 \\
\hline Group (SM) & $12.61[2.83,22.39]$ & 4.83 & 37.56 & 2.61 & $.013 *$ \\
\hline Time & $-1.89[-4.70, .92]$ & 1.36 & 22.23 & -1.38 & .177 \\
\hline $\begin{array}{l}\text { Group x time } \\
\text { (CPT) }\end{array}$ & $-.40[-4.67,3.89]$ & 2.07 & 24.37 & -.19 & .850 \\
\hline $\begin{array}{l}\text { Group } x \text { time } \\
\text { (SM) }\end{array}$ & $-3.90[-8.32, .52]$ & 2.14 & 24.71 & -1.82 & .081 \\
\hline \multicolumn{6}{|l|}{ Random effects } \\
\hline Intercept & $103.86[63.37,170.21]$ & \multicolumn{4}{|c|}{$<.001 * * *$} \\
\hline Baseline & $64.33[33.40,123.89]$ & \multicolumn{4}{|r|}{$.016^{*}$} \\
\hline Post-treatment & $53.50[23.71,120.69]$ & \multicolumn{4}{|r|}{$.003 * *$} \\
\hline Follow-up & $8.07[.38,172.68]$ & \multicolumn{4}{|r|}{.522} \\
\hline
\end{tabular}

Note. CPT $=$ Cognitive Processing Therapy, $\mathrm{SM}=$ Stress Management. Waitlist was used as a comparison groups. $* p<.05 . * * p<.01 . * * * p<.001$.

Table 20. Multilevel Model of DTS Absorption scores over time

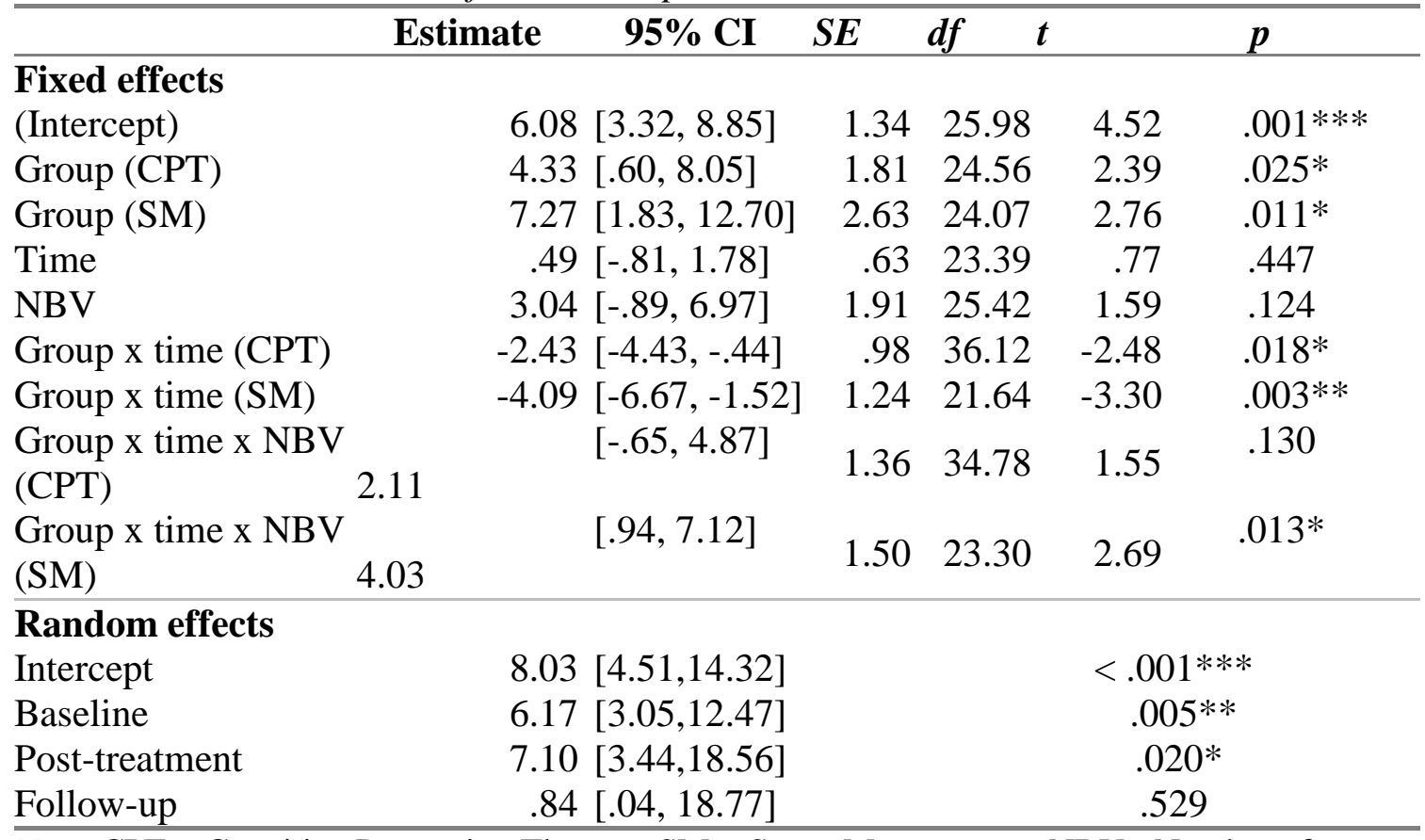

Note. CPT = Cognitive Processing Therapy, $\mathrm{SM}=$ Stress Management, NBV= Number of Bullying Victimization experiences. Waitlist was used as a comparison group. ${ }^{*} p<.05$. ${ }^{*} p<$ $.01 . * * *<.001$. 
Table 21. Multilevel Model of DTS Appraisal scores over time

\begin{tabular}{|c|c|c|c|c|c|c|}
\hline & Estimate & $95 \% \mathrm{CI}$ & $S E$ & $d f$ & $t$ & $p$ \\
\hline \multicolumn{7}{|l|}{ Fixed effects } \\
\hline Group (CPT) & $\begin{array}{r}1.01 \\
1.52\end{array}$ & {$[-4.75,7.79]$} & $\begin{array}{l}2.10 \\
3.09\end{array}$ & $\begin{array}{l}59.91 \\
36.71\end{array}$ & $\begin{array}{r}.43 \\
.49\end{array}$ & .627 \\
\hline Group (SM) & 5.94 & {$[-.46,12.35]$} & 3.16 & 37.33 & 1.88 & .068 \\
\hline Time & -1.27 & {$[-3.17, .64]$} & .92 & 22.03 & -1.38 & .182 \\
\hline Group x time (CPT) & .65 & {$[-2.25,3.55]$} & 1.40 & 22.46 & .46 & .647 \\
\hline Group x time (SM) & -1.71 & {$[-4.71,1.30]$} & 1.45 & 23.69 & -1.18 & .251 \\
\hline \multicolumn{7}{|l|}{ Random effects } \\
\hline Intercept & 33.17 & {$[19.86,55.39]$} & & & & $<.019 *$ \\
\hline Baseline & 15.52 & {$[6.86,35.11]$} & & & & $.016^{*}$ \\
\hline Post-treatment & 18.63 & {$[7.49,46.39]$} & & & & $.032 *$ \\
\hline Follow-up & 11.43 & {$[3.33,39.17]$} & & & & .112 \\
\hline
\end{tabular}

Note. CPT $=$ Cognitive Processing Therapy, $\mathrm{SM}=$ Stress Management. Waitlist was used as a comparison group. $* p<.05 . * * p<.01 . * * * p<.001$.

Table 22. Multilevel Model of DTS Regulation scores over time

\begin{tabular}{|c|c|c|c|c|c|c|}
\hline & Estimate & $95 \% \mathrm{CI}$ & SE & $d f$ & $t$ & $p$ \\
\hline \multicolumn{7}{|l|}{ Fixed effects } \\
\hline Group (CPT) & 1.37 & {$[-1.35,4.09]$} & 1.33 & 27.20 & 1.03 & .311 \\
\hline Group (SM) & 4.17 & {$[1.41,6.92]$} & 1.34 & 26.87 & 3.10 & $.005 * *$ \\
\hline Time & .11 & {$[-.86,1.09]$} & .47 & 20.64 & .24 & .813 \\
\hline Group x time (CPT) & .05 & {$[-1.39,1.50]$} & .70 & 22.75 & .08 & .939 \\
\hline Group x time (SM) & -1.17 & {$[-2.65, .32]$} & .72 & 23.56 & -1.62 & .119 \\
\hline \multicolumn{7}{|l|}{ Random effects } \\
\hline Intercept & 4.87 & {$[2.11,11.20]$} & & & & $<.019 *$ \\
\hline Baseline & 6.93 & {$[3.63,13.23]$} & & & & $.002 * *$ \\
\hline Post-treatment & 5.01 & {$[2.17,11.57]$} & & & & $.019 *$ \\
\hline Follow-up & 1.82 & {$[.37,8.96]$} & & & & .219 \\
\hline
\end{tabular}

Note. CPT $=$ Cognitive Processing Therapy, $\mathrm{SM}=$ Stress Management. Waitlist was used as a comparison group. ${ }^{*} p<.05 . * * p<.01 . * * * p<.001$. 
Table 23. Multilevel Model of DTS Tolerance scores over time

$\begin{array}{llllll}\text { Estimate } & 95 \% \mathrm{CI} & S E & d f & t & p\end{array}$

\section{Fixed effects}

(Intercept)

Group (CPT)

$10.29[7.59,12.98]$

$-.93[-4.46,2.60]$

$1.34 \quad 45.81$

$1.75 \quad 41.07$

$7.68 \quad .001 * * *$

Group (SM)

$6.54[1.43,11.66]$

.598

Time

$-.52[-1.57, .53]$

$2.58 \quad .013^{*}$

NBV

$-.52[-4.53,3.49]$

.50

$-.09[-1.78,1.60]$

1.99

.309

Group x time (CPT)

$-4.07[-6.17,-1.97]$

.83

17.29

$-1.05$

.797

Group x time (SM)

Group $x$ time $x$ NBV

(CPT)

$\begin{array}{llllll}-.16 & {[-2.45,2.13]} & 1.11 & 25.89 & -.14 & .887\end{array}$

$99 \quad 16.63$

.918

Group x time x NBV

$3.33[.82$,

$1.19 \quad 17.33$

2.79

$.012 *$

(SM) $5.84]$

\section{Random effects}

Intercept

11.09 [7.09,

Baseline

$3.20[1.22,8.28]$

$<.001 * * *$

$6.94[3.12$,

$.042 *$

Post-treatment

$.48[.00,41.57]$

$.014^{*}$

Follow-up

$.48[.00,41.57]$

.660

Note. CPT $=$ Cognitive Processing Therapy, $\mathrm{SM}=$ Stress Management, NBV= Number of

bullying victimization experiences. Waitlist was used as a comparison group. ${ }^{*} p<.05$. ${ }^{*} p<.01$. $* * * p<.001$. 


\subsubsection{Intolerance of uncertainty: IUS-12}

No significant effects were found in mean total IUS-12 scores for CPT or SM over time compared to the waitlist (see Table 24). Number of treatment sessions completed, time of bullying victimization experiences, and number of lifetime bullying victimization experiences did not produce significant effects or lead to improved fit in any of the models tested. Models did not converge for the IUS-12 Inhibitory subscale when time was treated as a repeated variable. Therefore, time was only treated as covariate when testing the effect of the predictors on the IUS-12 Inhibitory subscale. No significant effects were found in mean total scores on the Inhibitory subscale in CPT or SM over time compared to the waitlist. Number of treatment sessions completed, number of lifetime bullying victimization experiences, and time of bullying victimization experiences did not produce significant effects or improved fit in any of the models tested (see Table 25).

Mean total scores on the IUS-12 Prospective subscale increased over time in SM compared to the waitlist. SM was associated with a Cohen's $d$ of 0.73 (95\% CI [.08, 1.37]).No significant differences were found in CPT compared to the waitlist. Number of bullying victimization experiences was as significant predictor in SM, and mean total IUS-12 Prospective scores decreased more for participants that had a single bullying victimization experience compared to participants in SM that reported more than one bullying victimization experience and compared to the waitlist. This interaction was associated with a Cohen's $d$ of -0.68 (95\% CI [-1.45, .09]). Number of treatment sessions completed and time of bullying victimization experience did not produce significant effects or improve fit in any of the models tested (see Table 26). 
Table 24. Multilevel Model of IUS-12 total scores over time

\begin{tabular}{|c|c|c|c|c|c|}
\hline & Estimate & $S E$ & $d f$ & $t$ & $p$ \\
\hline \multicolumn{6}{|l|}{ Fixed effects } \\
\hline (Intercept) & $38.34[31.85,44.83]$ & 3.24 & 53.87 & 11.85 & $.001 * * *$ \\
\hline Group (CPT) & $-.37[-9.51,8.77]$ & 4.57 & 58.31 & -.08 & .936 \\
\hline Group (SM) & $-10.44[-19.76,-1.12]$ & 4.66 & 57.26 & -2.24 & $.029 *$ \\
\hline Time & $.27[-1.75,2.29]$ & .94 & 13.11 & .29 & .778 \\
\hline $\begin{array}{l}\text { Group x time } \\
(\mathrm{CPT})\end{array}$ & $-1.03[-4.28,2.22]$ & 1.54 & 16.74 & -.69 & .513 \\
\hline Group x time (SM) & $-1.74[-1.49,4.98]$ & 1.52 & 15.01 & 1.15 & .269 \\
\hline \multicolumn{6}{|l|}{ Random effects } \\
\hline Intercept & $126.63[80.01,200.43]$ & & & & $<.001 * * *$ \\
\hline Baseline & $68.75[38.99,121.24]$ & & & & $<.001 * *$ \\
\hline Post-treatment & $9.06[2.49,32.95]$ & & & & .129 \\
\hline Follow-up & $1.15[.00,9237.07]$ & & & & .827 \\
\hline
\end{tabular}

Note. CPT $=$ Cognitive Processing Therapy, $\mathrm{SM}=$ Stress Management. Waitlist was used as a comparison group. $* p<.05 . * * p<.01 . * * * p<.001$.

Table 25. Multilevel Model of IUS-12 Inhibitory scores over time

\begin{tabular}{lcrrrc}
\hline \multicolumn{1}{c}{ Estimate } & $\mathbf{9 5 \%} \mathbf{C I}$ & $\boldsymbol{S E}$ & $\boldsymbol{d} \boldsymbol{f}$ & $\boldsymbol{t}$ & $\boldsymbol{p}$ \\
Fixed effects & & & & & \\
Group (CPT) & $22.99[18.64,27.35]$ & 2.19 & 89.20 & 10.49 & $.001^{* * *}$ \\
Group (SM) & $-.87[-7.20,5.47]$ & 3.19 & 90.02 & -.27 & .786 \\
Time & $-7.40[-13.90,-.90]$ & 3.27 & 90.77 & -2.26 & $.026^{*}$ \\
Group x time (CPT) & $.89[-.89,2.67]$ & .88 & 36.36 & 1.02 & .317 \\
Group x time (SM) & $-.48[-3.20,2.25]$ & 1.34 & 37.64 & -.35 & .725 \\
Random effects & $-1.76[-1.05,4.59]$ & 1.39 & 39.01 & 1.27 & .213 \\
Intercept & $41.64[26.24,66.09]$ & & & & \\
Residual & $11.87[7.28,19.36]$ & & & & $<.001^{* * *}$ \\
\hline
\end{tabular}

Note. CPT $=$ Cognitive Processing Therapy, $\mathrm{SM}=$ Stress Management. Waitlist was used as a comparison group. $* p<.05 . * * p<.01 . * * * p<.001$. 
Table 26. Multilevel Model of IUS-12 Prospective scores over time

$\begin{array}{llll}\text { Estimate } & \text { 95\% CI } & S E & d f\end{array}$

Fixed effects

(Intercept)

Group (CPT)

Group (SM)

Time

NBV

$16.97[13.19,20.76]$

$-3.87[-8.70, .97]$

1.89

60.68

$\begin{array}{lll}2.42 & 61.36 & -1.60\end{array}$

$53.21-2.93$

$-10.40[-17.52,-3.29]$

3.55

$-.91[-2.24, .42]$

$\begin{array}{lll}.62 & 13.02 & -1.47\end{array}$

$-4.71[-10.49,1.06]$

2.88

$57.81-1.64$

$1.63[1.54,6.91]$

$\begin{array}{lll}1.24 & 39.79 & 1.43\end{array}$

$4.22[1.54,6.91]$

$\begin{array}{lll}3.42 & 12.29 & 3.42\end{array}$

$-2.66[-5.63, .32]$

1.46

33.42

$-1.8$

$t \quad p$

Group $x$ time $\mathrm{x}$ NBV

(CPT)

$\begin{array}{llllll}-4.43 & {[-7.63,-1.24]} & 1.48 & 13.12 & -2.99 & .010^{* *}\end{array}$

Group x time x NBV

(SM)

\section{Random effects}

Intercept

Baseline

Post-treatment

Follow-up

$$
\begin{aligned}
25.15 & {[16.17,39.11] } \\
8.50 & {[4.36,16.52] } \\
2.73 & {[.91,8.22] } \\
.21 & {[5.52,} \\
& 8280.69]
\end{aligned}
$$$$
<.001 * * *
$$$$
<.003 * *
$$$$
.075
$$

.853

Note. CPT $=$ Cognitive Processing Therapy, $\mathrm{SM}=$ Stress Management, NBV= Number of

bullying victimization experiences. Waitlist was used as a comparison group. $* p<.05$. $* * p .01$. $* * * p<.001$. 


\subsubsection{Anxiety sensitivity: ASI-3}

Mean total scores on the ASI-3 scale increased in SM compared to the waitlist. SM was associated with a Cohen's $d$ of 0.48 (95\% CI $[.00, .95])$. No significant differences were found in CPT compared to the waitlist. However, number of lifetime bullying victimization experiences was a significant predictor in CPT, and mean total ASI-3 scores decreased significantly more for participants in CPT who had a single bullying victimization experience compared to CPT participants with more than one bullying victimization experience and compared to the waitlist (see Table 27). This interaction was associated with a Cohen's $d$ of -0.49 (95\% CI [-.99, 01]). Number of treatment sessions completed and time of bullying victimization experiences did not produce significant effects or improved fit in any of the models tested.

Mean total scores on the ASI-3 Physical Concerns subscale increased in CPT compared to the waitlist (see Table 28). CPT was associated with a Cohen's $d$ of 0.69 (95\% CI $[.12,1.26])$. No significant differences were found in mean total ASI-3 Physical Concerns scores in SM compared to the waitlist. Time of bullying victimization experiences was a significant predictor in CPT, and total mean scores on the ASI-3 Physical Concerns subscale decreased significantly more for participants in CPT with a remote bullying experience compared to CPT participants with a recent bullying victimization experience and compared to the waitlist. This interaction was associated with a Cohen's $d$ of -0.95 (95\% CI [-1.58, -.33]). Number of treatment sessions completed and number of lifetime bullying victimization experiences did not produce significant effects or improve fit in any of the models tested. Pairwise comparisons were made between measurement occasions in the models for the ASI-3 Cognitive Concerns 
subscale as time was a significant covariate. Mean total ASI-3 Cognitive scores decreased significantly from baseline to follow-up across all groups (see Table 29). No significant time or treatment group interaction effects were found for in ASI-3 Cognitive Concerns scores.

Models did not converge for the ASI-3 Social Concerns scores when time was treated as a repeated variable. Pairwise comparisons were made between measurement occasions in the models for ASI-3 Social Concerns, as time was a significant covariate. Mean total scores on the ASI-3 Social Concerns subscale decreased significantly from baseline to post-treatment in SM, and from baseline to follow-up. SM was associated with a Cohen's $d$ of -0.82 at post-treatment, and -0.62 at follow-up. No significant differences were found in ASI-3 Social Concerns scores in CPT compared to the waitlist (see Table 30). Number of treatment sessions completed, time of bullying victimization experiences and number of lifetime bullying victimization experiences did not produce significant effects or improve fit in any of the models tested. 
Table 27. Multilevel Model of ASI-3 total scores over time

\begin{tabular}{|c|c|c|c|c|c|}
\hline & Estimate & $S E$ & $d f$ & $t$ & $p$ \\
\hline $\begin{array}{l}\text { Fixed effects } \\
\text { (Intercept) }\end{array}$ & $44.67[34.46,54.88]$ & 5.14 & 87.16 & 8.70 & $.001 * * *$ \\
\hline Group (CPT) & $-13.47[-26.65,-2.85]$ & 6.64 & 90.99 & -2.03 & $.045^{*}$ \\
\hline Group (SM) & $-20.21[-39.38,-1.03]$ & 9.65 & 90.10 & -2.09 & $.039 *$ \\
\hline Time & $-.23[-3.60,3.13]$ & 1.66 & 34.75 & -1.03 & .308 \\
\hline NBV & $-8.04[-23.64,7.56]$ & 7.84 & 77.36 & -1.03 & .308 \\
\hline $\begin{array}{l}\text { Group } x \text { time } \\
(\mathrm{CPT})\end{array}$ & $3.20[-2.47,8.87]$ & 2.83 & 51.72 & 1.13 & .263 \\
\hline Group x time $(\mathrm{SM})$ & $6.94[.03,13.86]$ & 3.41 & 35.26 & 2.04 & $.049 *$ \\
\hline $\begin{array}{l}\text { Group } \times \text { time } x \\
\text { NBV }(\mathrm{CPT})\end{array}$ & $-7.18[-14.50,-.14]$ & 3.63 & 44.63 & -1.98 & $.054^{*}$ \\
\hline $\begin{array}{l}\text { Group } \times \text { time } x \\
\text { NBV }(\mathrm{SM})\end{array}$ & $-7.73[-15.95, .48]$ & 4.05 & 35.19 & -1.91 & .064 \\
\hline \multicolumn{6}{|l|}{ Random effects } \\
\hline Intercept & $176.51 \quad[118.68,262.52]$ & & & & $\leq .001 * * *$ \\
\hline Residual & {$[11.34,29.91]$} & & & & $\leq .001 * * *$ \\
\hline
\end{tabular}

Note . $\mathrm{CPT}=$ Cognitive Processing Therapy, $\mathrm{SM}=$ Stress Management, $\mathrm{NBV}=$ Number of

bullying victimization experiences. Waitlist was used as a comparison group. ${ }^{*} p<.05 .{ }^{* *} p<.01$.

$* * * p<.001$.

Table 28. Multilevel Model of ASI-3 Physical scores over time

\begin{tabular}{|c|c|c|c|c|c|}
\hline & Estimate & $S E$ & $d f$ & $t$ & $p$ \\
\hline Fixed effects & $1668[8.73,2463]$ & 400 & 8669 & 4.17 & $001 * * *$ \\
\hline Group (CPT) & $-8.95[-19.49,1.58]$ & 5.30 & 90.23 & -1.69 & .095 \\
\hline Group (SM) & $-5.72[-19.05,7.60]$ & 6.70 & 75.65 & -.86 & .395 \\
\hline Time & $-2.32[-4.87, .22]$ & 1.25 & 35.62 & -1.85 & .072 \\
\hline TBV & $-4.57[-13.28,4.15]$ & 4.38 & 85.35 & -1.04 & .300 \\
\hline Group x time (CPT) & $4.36[.77,7.95]$ & 1.77 & 35.82 & 2.46 & $.019 *$ \\
\hline Group x time (SM) & $2.76[-2.91,8.43]$ & 2.80 & 36.04 & .98 & .330 \\
\hline $\begin{array}{l}\text { Group } \times \text { time } x \\
\text { TBV }(\mathrm{CPT})\end{array}$ & $-6.02[-9.96,-2.09]$ & 1.94 & 35.77 & -3.11 & $.004 * *$ \\
\hline $\begin{array}{l}\text { Group } \times \text { time } x \\
\text { TBV }(\text { SM })\end{array}$ & $-3.14[-9.04,2.75]$ & 2.91 & 36.02 & -1.09 & .287 \\
\hline \multicolumn{6}{|l|}{ Random effects } \\
\hline Intercept & $32.57[22.00,48.21]$ & & & & $\leq .001 * * *$ \\
\hline Residual & $3.25[2.01,5.24]$ & & & & $\leq .001 * * *$ \\
\hline
\end{tabular}

Note . CPT $=$ Cognitive Processing Therapy, $\mathrm{SM}=$ Stress Management, $\mathrm{TBV}=$ Time of bullying victimization experiences. Waitlist was used as a comparison group. $* p<.05$. $* * p<.01$. $* * * p<$ .001 . 
Table 29. Multilevel Model of ASI-3 Cognitive scores over time

\begin{tabular}{|c|c|c|c|c|c|}
\hline & Estimate & $S E$ & $d f$ & $t$ & $p$ \\
\hline \multicolumn{6}{|l|}{ Fixed effects } \\
\hline (Intercept) & $13.38 \begin{array}{c}{[10.92,} \\
15.83]\end{array}$ & 1.23 & 66.10 & 10.86 & $.001 * * *$ \\
\hline Group (CPT) & $-3.24[-6.45,-.04]$ & 1.60 & 66.10 & -2.02 & $.047 *$ \\
\hline Group (SM) & $-2.08[-5.45,1.28]$ & 1.68 & 66.23 & -1.24 & .220 \\
\hline Post-tx & $-.82[-3.15,1.51]$ & 1.16 & 42.59 & -.71 & .482 \\
\hline $\mathrm{Fu}$ & $-3.97[-6.59,-1,34$ & 1.30 & 38.52 & -3.05 & $.004 * *$ \\
\hline $\begin{array}{l}\text { Group x post-tx } \\
\text { (CPT) }\end{array}$ & $.14[-3.47,3.75]$ & 1.79 & 42.79 & .07 & .939 \\
\hline Group x fu (CPT) & $1.28[-2.63,5.19]$ & 1.94 & 40.09 & .66 & .511 \\
\hline $\begin{array}{l}\text { Group x post-tx } \\
(\mathrm{SM})\end{array}$ & $-2,48[-6.11,1.15]$ & 1.80 & 47.49 & -1.38 & .175 \\
\hline Group x fu (SM) & $2.24[-1.78,6.27]$ & 2.00 & 43.19 & 1.13 & .267 \\
\hline \multicolumn{6}{|l|}{ Random effects } \\
\hline Intercept & $12.06[7.09,20.50]$ & & & & $\leq .001 * * *$ \\
\hline Baseline & $12.22[7.06,21.17]$ & & & & $<.000 * * *$ \\
\hline Post-treatment & $1.53[.18,12.76]$ & & & & .355 \\
\hline Follow-up & $3.65[1.33,9.97]$ & & & & $.051 *$ \\
\hline
\end{tabular}

Table 30. Multilevel Model of ASI-3 Social scores over time

\begin{tabular}{|c|c|c|c|c|c|c|}
\hline & Estimate & $95 \% \mathrm{CI}$ & $S E$ & $d f$ & $t$ & $p$ \\
\hline $\begin{array}{l}\text { Fixed effects } \\
\text { (Intercept) }\end{array}$ & 16.29 & {$[13.04,19.53]$} & 1.62 & 61.04 & 10.04 & $.001 * * *$ \\
\hline Group (CPT) & -1.46 & {$[-5.66,2.73]$} & 2.10 & 59.62 & -.70 & .488 \\
\hline Group (SM) & -.60 & {$[-4.98,3.78]$} & 2.19 & 59.95 & -.27 & .786 \\
\hline Post-tx & .58 & {$[-1.09,2.25]$} & .82 & 34.07 & .70 & .486 \\
\hline $\mathrm{Fu}$ & -1.29 & {$[-3.05, .48]$} & .87 & 34.03 & -1.48 & .147 \\
\hline $\begin{array}{l}\text { Group x post-tx } \\
(\mathrm{CPT})\end{array}$ & -1.65 & {$[-4.46,1.17]$} & 1.39 & 34.29 & -1.19 & .244 \\
\hline Group x fu (CPT) & -.37 & {$[-3.09,2.34]$} & 1.34 & 34.32 & -.28 & .782 \\
\hline $\begin{array}{l}\text { Group x post-tx } \\
\text { (SM) }\end{array}$ & -5.39 & {$[-8.16,-2.62]$} & 1.36 & 34.82 & -3.95 & $.000 * * *$ \\
\hline Group x fu (SM) & -4.09 & {$[-6.91,-1,26]$} & 1.39 & 34.77 & -2.94 & $.006 * *$ \\
\hline \multicolumn{7}{|l|}{ Random effects } \\
\hline Intercept & 38.79 & $\begin{array}{l}{[26.38} \\
57.03]\end{array}$ & & & & $<.001 * * *$ \\
\hline Residual & 2.76 & {$[1.70,4.47]$} & & & & $<.001 * * *$ \\
\hline
\end{tabular}

Note. CPT $=$ Cognitive Processing Therapy, $\mathrm{SM}=$ Stress Management, Post-tx $=$ Post-Treatment, $\mathrm{Fu}=$ Follow-up. Waitlist was used as a comparison group. $* p<.05 . * * p<.01 . * * * p<.001$. 


\subsection{Clinically Significant Changes}

Examination of changes in participants' total scores from baseline to posttreatment, and one-month follow-up in CPT and SM revealed clinically significant symptom reduction on most measures. However, given the small number of data points at post-treatment and follow-up due to the intent-to-treat design of the trial (i.e., participants had to have completed one treatment session to be included in the primary analyses) and large participant drop-out (see above how HLM handles missing data), these findings are interpreted with caution. Seven participants in CPT had complete data for the baseline, post-treatment and follow-up measurement occasions. Thereof, PTSD symptoms decreased in clinically meaningful ways (i.e., went from above the clinical cut-off total score of 30 or higher on the PCL-5 to below 30) for $45.0 \%$ from baseline to posttreatment, and for $57.0 \%$ from baseline to follow-up. Strength of maladaptive appraisals decreased in clinically meaningful ways (i.e., total scores decreased from the above 133 range on the PTCI) for $43.9 \%$ of participants from baseline to post-treatment, and from baseline to follow-up. On the SIPS, $29.0 \%$ of the seven CPT participants had clinically significant changes from baseline to post-treatment and baseline to follow-up (i.e., total scores went from 21 or higher to below 21 on the SIPS). Severity of depression decreased for $43.0 \%$ of these participants (i.e., total scores went from the extremely severe, severe or moderate range to below 14 on the DASS-21 Depression subscale), and severity of stress decreased for $14.3 \%$ (i.e., total scores from the extremely severe, severe or moderate range to below 19), while severity of anxiety did not change in clinically meaningful ways (i.e., severity of anxiety remained in the moderate to severe range). Anger decreased below the clinical cut-off (i.e., 12 or higher on the DAR-5) for $28.6 \%$ of 
the seven CPT participants from baseline to post-treatment, and for $43.9 \%$ from baseline to follow-up. Six participants in SM had complete data for the baseline, post-treatment and follow-up measurement occasions. Symptoms of PTSD reduced out of the clinically significant range for $33.0 \%$ of these participants (i.e., went from above the clinical cut-off total score of 30 or higher on the PCL-5 to below 30) from baseline to post-treatment, and $33.0 \%$ from baseline to follow-up. Strength of maladaptive appraisals decreased in clinically significant ways (i.e., total scores decreased from the above 133 range on the PTCI) for $16.7 \%$ of the six SM participants from baseline to post-treatment, and $16.7 \%$ from baseline to follow-up. Severity of depression, anxiety, stress, and anger decreased from the clinically significant ranges for $16.7 \%$ of the participants from baseline to posttreatment, and $16.7 \%$ from baseline to follow-up. Scores on the ASI-3, IUS-short and DTS remained in the high and moderate levels at post-treatment and follow-up in both CPT and SM.

\subsection{Secondary Analyses}

Hypothesis 3 and 4 were tested using multiple regressions. Hypothesis 3 stated that resilience, social support, and coping would mediate the relationship between event centrality and strength of maladaptive appraisals. Hypothesis 3 stated that the number of reported bullying victimization experiences would moderate the impact of maladaptive appraisals on psychological distress (i.e., as measured by the DASS-21, PCL-5, SIPS, and DAR-5).

For hypothesis 3, a simple regression testing the direct effect of event centrality on the strength of maladaptive appraisals produced significant results $(b=1.77,95 \% \mathrm{CI}$ $[.90,2.65], p \leq .001)$. No significant effects were found between event centrality and 
resilience $(b=.04,95 \%$ CI $[-.13,2.1], p=.664)$ or centrality and social support $(b=-$ $0.45,95 \%$ CI $[-.96, .07], p=.089)$. The effect of event centrality was tested for each of the nine CERQ subscales, assessing cognitive emotion regulation. Event centrality was not a significant predictor of self-blame $(b=.01,95 \%$ CI $[-.06, .09], p=.733)$, acceptance $(b=-0.00,95 \%$ CI $[-.07, .06], p=.929)$, positive refocusing $(b=-0.05,95 \%$ CI $[-.12, .02], p=.138)$, or putting into perspective $(b=-0.05,95 \%$ CI $[-.12, .02], p$ $=.124)$. Significant effects were, however, found for the relationship between event centrality and rumination $(b=0.12,95 \%$ CI $[.05, .18], p \leq .001)$, planning, $(b=0.08$, $95 \%$ CI $[.03, .13], p \leq .01)$, positive reappraisal $(b=0.06,95 \%$ CI $[.00, .12], p \leq .038)$, catastrophizing $(b=0.16,95 \%$ CI $[.10, .21], p \leq .001)$, and other blame $(b=0.12,95 \%$ CI $[.05, .19], p \leq .001)$. Finally, the effect of event centrality and each of the significant proposed mediation variables was tested using multiple regression. Results indicated that the relationship between event centrality and maladaptive appraisals was mediated by catastrophizing $\left(b=5.13,95 \%\right.$ CI $\left.[.71,9.54], p \leq .02, R^{2}=.34\right)$. Figure 5 depicts this interaction.

For hypothesis 4, simple regressions were completed testing the effect of maladaptive appraisals (i.e., PTCI total scores) on psychological distress (i.e., total scores on the SIPS, DAR-5, PCL-5, and DASS-21). Significant associations were found between maladaptive appraisals and SIPS total scores $(b=0.25,95 \%$ CI $[.16,34], p$ $\left.\leq .001, R^{2}=.35\right)$, DASS-21 total scores $\left(b=0.25,95 \%\right.$ CI $\left.[.13,31], p \leq .001, R^{2}=.31\right)$, DAR-5 scores $\left(b=0.08,95 \%\right.$ CI $\left.[.05,11], p \leq .001, R^{2}=.33\right)$, and PCL-5 scores $(b=$ $0.34,95 \%$ CI $\left.[.23,44], p \leq .001, R^{2}=.42\right)$. Next, the bivariate correlation between maladaptive appraisals and the moderator variable, number of bullying victimization 
experiences, was examined. The correlation between maladaptive appraisals and number of bullying victimization experiences was $R=.59$, but did not reach statistical significance $p=.07$. Multiple regressions were, nevertheless, conducted to examine the combined effect of maladaptive appraisals and number of bullying victimization experiences. Number of bullying victimization experiences was not a unique predictor of SIPS total scores $\left(b=1.84,95 \%\right.$ CI $\left.[-3.86,7.55], p=.520, R^{2}=.37\right)$, DASS-21 scores $(b$ $=0.49,95 \%$ CI $\left.[-5.13,6.11], p=.862, R^{2}=.32\right)$, or PCL-5 scores $(b=5.77,95 \%$ CI [-.84, $\left.12.38], p=.086, R^{2}=.46\right)$. However, number of bullying victimization experiences was a unique predictor in the relationship between maladaptive appraisals and DAR-5 scores $(b$ $=2.12,95 \%$ CI $\left.[.26,3.99], p \leq .05, R^{2}=.38\right)$. The combined effect of maladaptive appraisals and number of bullying victimization experiences on DAR-5 scores was greater than the effect of maladaptive appraisals on DAR-5 scores alone $\left(R^{2}=.40\right.$ compared to $R^{2}=.33$ ) suggesting a moderation effect (see Figure 6). 


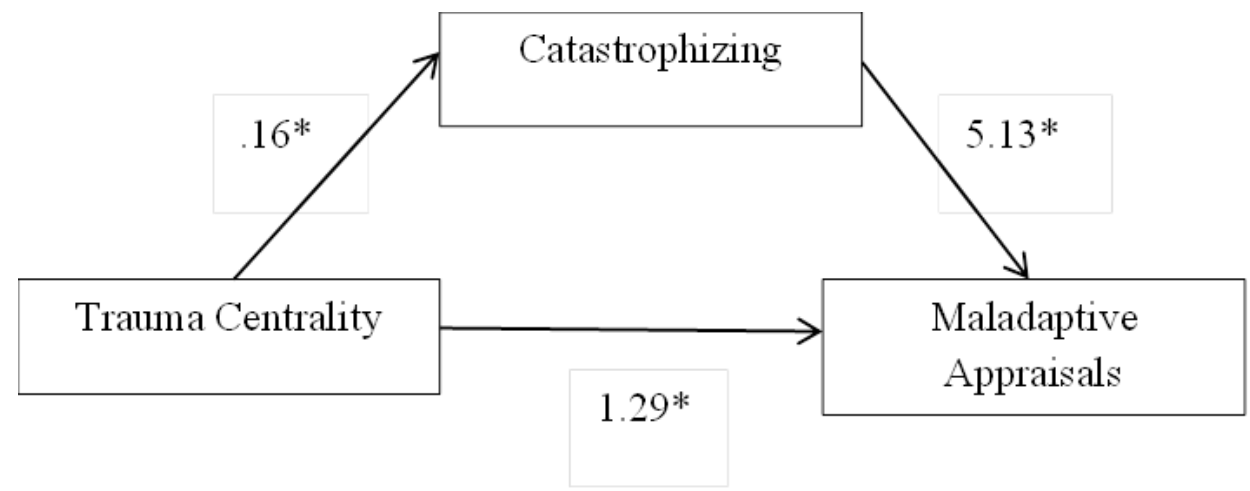

Figure 5. Mediation effect of catastrophizing on the relationship between event centrality and maladaptive appraisals. Note: ${ }^{*} p \leq .05$

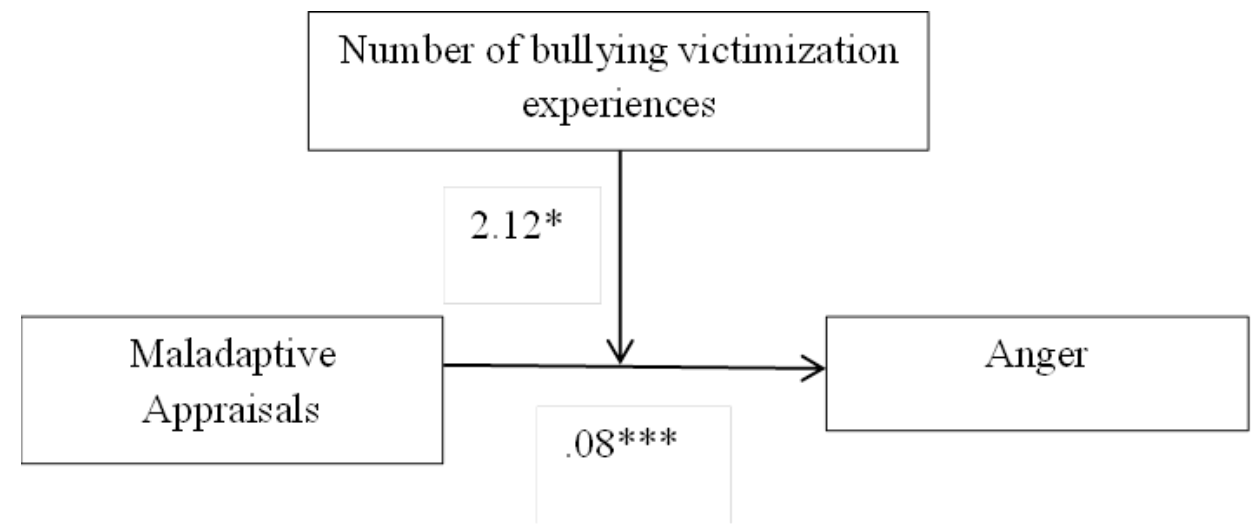

Figure 6: Moderation effect of number of bullying victimization experiences on the relationship between maladaptive appraisals and anger. Note: ${ }^{*} p \leq .05 ;{ }^{*} p \leq .001$ 


\section{DISCUSSION}

Bullying victimization is associated with pervasive and long-term negative psychosocial outcomes; yet, no interventions exist that directly focus on updating the specific impact of the experience and associated memories on functioning. Bullying is in many ways synonymous to trauma in that victims frequently experience symptoms of PTSD, including maladaptive appraisals about themselves and others, intrusions, avoidance, and hyperarousal. The focus of the current trial was, therefore, to test a modified version of an internet-delivered CPT to target maladaptive trauma appraisals and associated symptoms of psychological distress arising from bullying victimization against an active control group and a waitlist.

Hypotheses 1 and 2 stated that participants receiving internet-delivered CPT would experience significantly greater improvement in trauma-related maladaptive appraisals and psychological distress over time compared to participants receiving internet-delivered SM and participants on the waitlist. Hypotheses 1 and 2 were partially supported. CPT lead to greater reductions on some of the outcome measures compared to the waitlist and SM; but, SM led to comparable symptom improvements to CPT on other measures (see below). Number of lifetime bullying victimization experiences reported by participants, number of treatment sessions completed, and time of bullying victimization significantly impacted the effectiveness of the treatments on both maladaptive appraisals and measures of psychological distress. Specifically, outcomes were generally better for participants who endorsed a single lifetime bullying victimization experience than multiple bullying victimization experiences, for participants who completed more 
treatment sessions, and for participants with remote bullying victimization compared to a recent experience.

Twenty-two participants completed the treatment content surveys. Thereof, 11 had been randomized to CPT and 11 to SM. Findings of the content surveys indicated that participants' overall impression was more favorable for CPT than SM. Participants from the CPT group reported finding the treatment and therapist support helpful, that they would recommend the treatment to others, and that the length of the program was either adequate or too short. Participants in the SM group also reported finding the program and therapist support helpful, but deemed the length of program as either adequate or too long. Furthermore, several participants in the SM group reported dropping out of treatment because the program was slightly too long or was not perceived as helpful, while no participants indicated discontinuing CPT for these reasons. Taken together, these findings are in line with previous research on acceptability of internet-delivered cognitive behavioural therapy, which indicates that participants generally have a favorable impression of the therapy experience, but report finding the time allocated to learning and practicing skills before moving on to the next treatment module too short (Hadjistavropoulos, Faller, Klatt, Nugent, Dear, \& Titov, 2018). Although the findings of the content survey in this trial provide insight into participant perceptions of the CPT and SM programs, the small number of survey responders undermines the overall conclusions. Nevertheless, attrition rates were approximately $20 \%$ higher in SM compared to CPT, which suggests that perceptions as reflected in the content survey were generally consistent with the sample at large. 
Mean total scores on the three measures that were administered weekly throughout the trial - the SIPS, DASS-21, and DAR-5-decreased gradually over time in both CPT and SM; however, the pattern of symptom change in SM was linear whereas the pattern of change in CPT was somewhat quadratic (i.e., with symptoms remaining at consistent levels and increasing before decreasing). These findings are in line with previous research on change patterns in trauma-focused treatment of anger and PTSD symptoms, which suggest that improvements in both constructs follow both quadratic and linear patterns (Nishith et al., 2002; Resick et al., 2008). The different change patterns observed in the weekly symptom measures might also reflect the differences in attrition in SM and CPT. Participants in SM whose symptoms did not change or increased as treatment progressed might have been more likely to drop out of treatment than those who experienced steady symptom changes because they believed treatment was not helping. Conversely, participants in CPT might have been more comfortable experiencing symptom increases because they were directly focusing on painful thoughts, emotions, and memoires from their bullying victimization experiences, and continued treatment in the hopes of symptom reduction as they perceived the treatment as more credible than participants in SM (Alfonsson Olsson, \& Hursti, 2016).

Treatment dropout in the internet-delivered CPT in this trial (i.e., 33\%) was comparable to previous research on in-person trauma-focused treatment which varies from 15 - 30\% between samples (Forbes et al., 2012; Imel, Laska, Jakupcak, \& Simpson, 2015; Rizvi, Vogt, \& Resick, 2009), and iCBT, where attrition is generally between 13 49\% (Banerjee \& Severn, 2018). Research on attrition in internet-delivered interventions has highlighted several factors that make treatment completion more likely, including 
older participant age (Edmonds, Hadjistavropoulos, Schneider, Dear, \& Titov, 2018) and increased frequency and quality of therapist support (i.e., therapist availability, use of evidence based treatment techniques; Alfonsson, Olsson, Linderman, Winnerhed, \& Hurst, 2016; Johansson \& Andersson, 2012). In the current trial, participants' age did not differ between treatment-completers and non-completers in both CPT and SM (see Table 1), but the frequency of therapist support was slightly lower than in previous studies (Alfonsson et al., 2016), which might have affected participant attrition. Another reason participants do not complete the full course of treatment, which might explain the attrition in this study, is that they experience sufficient improvement in functioning or distress before the end of treatment. In Galovski et al.'s (2012) study on varying lengths of CPT, $58 \%$ of participants achieved their end-state criterion (i.e., predetermined symptom improvement) before session 12 (medium session was 7.5).

\subsection{Summary of Outcomes}

\subsubsection{Maladaptive appraisals. Both CPT and SM were associated with} significant increases in PTCI total scores and Negative Self-Beliefs subscale scores over time, indicating that neither treatment was helpful for targeting maladaptive appraisals associated with bullying victimization. However, when number of bullying victimization experiences were included in the analyses there were reductions in total PTCI score and Negative Self-Beliefs subscale scores, with CPT outperforming the waitlist and SM in both cases. Participants in CPT who endorsed experiencing a single bullying victimization experience reported large reductions in maladaptive appraisals over time (i.e., Cohen's $d$ s of -0.84 and -0.86). With regards to the PTCI Negative Other-Beliefs subscale, both CPT and SM were moderately effective in reducing mean scores over time, 
but only for participants that endorsed a single bullying victimization experience (i.e., Cohens' $d$ of -0.60 in CPT and -0.73 in SM).

The different outcomes in maladaptive appraisals between participants who endorsed a single compared to more than one bullying victimization experience are consistent with the research literature on trauma and bullying victimization. Prior history of trauma increases the risk of maladaptive outcomes and predicts negative post-trauma appraisal (Ehlers \& Clark, 2000; Resick et al., 2014). Similarly, repeated bullying victimization experiences reinforce negative beliefs about self and others (e.g., "I will always be rejected by people"), and these beliefs can become embedded in an individual's core beliefs system. Eliciting and challenging deep rooted core beliefs is more difficult in cognitive therapy than more transient automatic beliefs (Wenzel, 2012); therefore, participants with multiple bullying victimization experiences may have needed a greater number of treatment sessions than offered in this trial to reduce the strength of their maladaptive beliefs.

The results of the analyses for the PTCI Self-Blame subscale differed from those found for the total scale and the other two subscales. Specifically, mean total scores on the PTCI Self-Blame subscale increased over time in SM compared to the waitlist (Cohen's $d$ of 0.68 ) and no significant changes were found in PTCI Self-Blame scores for participants in CPT. These findings are surprising given that CPT, and to some extent SM, was effective in reducing overall PTCI scores and scores on the Negative Self-Beliefs and Negative Other-Beliefs subscales for participants with a single bullying victimization experience. The psychometric properties of the PTCI Self-Blame subscale were, furthermore, adequate. Suppressor effects could possibly explain the findings for the 
PTCI Self-Blame subscale. Beck et al. (2004) found that the Self-Blame subscale had poor concurrent and discriminant validity compared to the whole scale and the other subscales. Similarly, Startup, Makgekgenene, and Webster (2007) found that, in contrast to the Negative Self-Beliefs and Negative Other-Beliefs subscales, higher scores on the Self-Blame subscale were associated with lower symptoms of posttraumatic stress, despite participants in their sample with PTSD having higher means on the scale compared to participants without PTSD. They suggested suppressor effects with items on the Negative Self- Beliefs subscale concealing the association between posttraumatic stress and scores on the Self-Blame subscale. Another possible explanation is that the Negative Self-Beliefs subscale more accurately measures internalized self-blame associated with PTSD than the Self-Blame scale.

4.1.2. Psychological Distress. Both CPT and SM were effective in reducing psychological distress; but, the treatments related differently to each of the psychological constructs measured. CPT was more effective for some of the symptom scales, whereas SM was more effective for others. Number of bullying victimization experiences, time of bullying victimization, and, to some extent, number of treatment sessions completed were significant predictors that impacted the effect of treatments on the psychological constructs.

CPT lead to large reductions in PTSD symptoms over time (Cohen's $d=-0.86$ ) compared to the waitlist, whereas SM did not have a significant effect on PTSD symptoms. These findings are in line with a large body of research supporting the effectiveness of CPT in reducing PTSD when compared to both in-active and active control conditions (Asmundson et al., 2019; Resick \& Schnicke, 1992; Resick et al., 
2002; Resick et al., 2008; Zappert \& Westrup, 2008). However, previous research has also found that relaxation techniques, such as those incorporated into the SM program, effectively reduce the global severity of PTSD symptoms, which is in contrast to the findings of this trial (Marks et al., 1998; Taylor et al. 2003).

Neither CPT nor SM reduced SIPS total scores. Participants in CPT reported a small increase in mean scores over time on the SIPS SIA (Cohen's $d=0.30$ ), and the SIPS SAA subscales (Cohen's $d=0.29$ ), but no change on the SIPS FOE subscale. SM was associated with a moderate increase in mean scores on the SIPS FOE subscale over time (Cohen's $d=0.50$ ), but did not lead to significant changes in scores on the SIPS SIA and SIPS SAA subscales. These findings contrast previous research suggesting that imagery rescripting, a technique which involves updating the meaning of faulty memories through imagery, and applied relaxation are both effective for reducing symptoms of social anxiety (Heimberg, 2002; Nilsson et al., 2012). Imagery rescripting is similar to the written trauma account in CPT in that both techniques are exposure based and aim at accessing, rather than avoiding, difficult thoughts and emotions associated with trauma or negative life experiences (Nilsson, et al., 2012, Resick et al., 2014). Cognitive therapies that utilize cognitive restructuring techniques similar to those applied in CPT have, furthermore, been found effective in reducing symptoms of SAD (Heimberg, 2002). A possible explanation for no changes in the CPT group is that the focus of the cognitive restructuring techniques was explicit to beliefs regarding self and others from bullying victimization experiences. Hofmann (2004) found that the strength of beliefs regarding estimated social cost mediated symptom reduction of social anxiety in cognitive behavioural therapy. The generalization of skills learned in CPT in this trial might not 
have encompassed beliefs regarding estimated cost in social situations or, perhaps, longer time was needed for the skills learned in CPT to generalize across more situations. Another possible reason for the lack of treatment effect on symptoms of social anxiety in this trial is that the intervention was delivered through the internet as opposed to inperson. Stronger therapeutic alliance is associated with better outcomes in CBT for SAD (Hays, Hope, VanDyke, \& Heimberg, 2007), and face-to-face intervention offers opportunities for modification to the individual needs of the client, such as in terms of motivation for treatment, which could lead to better alliance compared to internet interventions. Furthermore, clinicians seeing clients in-person can offer feedback about interpersonal skills and provide opportunities to practice skills that the client perceives as defective (Schulz, Vicent, \& Berger, 2017; Woods, Stults, Terry, \& Rego, 2017). Conversely, however, research also indicates that the strength of the therapeutic alliance in internet-delivered and therapist-guided treatments for depression and anxiety is comparable to alliance in treatment delivered in-person (Hadjistavropoulos, Pugh, Hesser, \& Andersson, 2016; Pihlaja et al., 2018).

SM had a large effect on total DASS-21 scores (Cohen's $d=-1.06)$ and DASS-21 Anxiety scores (Cohen's $d=-1.10$ ) and a small effect on DASS-21 Depression scores (Cohen's $d=-0.02$ ) compared to the waitlist. CPT had a small effect on DASS-21 total scores (Cohen's $d=-0.10)$ and DASS-21 Anxiety scores (Cohen's $d=-0.14$ ) for participants who completed more treatment sessions (i.e., number of treatment sessions completed was a significant predictor), but no effect on DASS-21 Depression scores. No significant treatment effects were found for the DASS-21 Stress subscale. These findings contrast previous research indicating that CPT is effective for both PTSD and concurrent 
symptoms of depression (Asmundson et al., 2019) and that relaxation effectively reduces depression, albeit to a lesser extent than cognitive behavioural therapies (Jorm, Morgan, \& Hetrick, 2008). The lack of effect of CPT on DASS-21 scores could be related to the quadratic pattern of symptom change found in the session-to-session analyses. Scores on the DASS-21 remained consistent and increased before they began to decrease after session nine of CPT. Several participants in the content survey who completed CPT reported that they found the intervention too short; so, it is possible that further improvement in DASS-21 scores would have been found if the treatment had included more sessions. Supporting this notion are findings from previous research that suggest that changes in PTSD frequently precede changes in depression symptoms over time in cognitive behavioural treatments for PTSD (Aderka, Foa, Applebaum, Shafran, \& Gilboa-Schechtman, 2011).

CPT and SM did not have significant effects on DAR-5 scores over time compared to the waitlist. This lack of treatment effect of CPT on anger in this trial is surprising given that PTSD symptoms reduced significantly in CPT, and previous research indicates that trauma-focused cognitive behavioural treatments are effective in reducing comorbid anger and PTSD (Cahill, Rauch, Hembree, \& Foa, 2003; Resick et al., 2008). The DAR-5 is, furthermore, sensitive to changes in anger from PTSD treatment (Forbes et al., 2004). Anger is, however, a multifaceted concept which encompasses not only anger disposition and anger towards others, which are the aspects of the construct primarily measured by the DAR-5, but also internalized anger and state anger (i.e., distinct episodes of anger; Deffenbacher et al., 1996; Forbes et al., 2004). The effect of PTSD treatment on anger may vary based on the anger construct assessed. Galovski, 
Elwood, Blain, and Resick (2014) compared changes in the anger dimensions (i.e., trait or state anger) and anger expression (i.e., inward, outward, anger control) based on responsivity to CPT for PTSD in a sample of female survivors of interpersonal violence. The rate of change in state anger and anger directed inward did not differ across participants who experienced significant gains in treatment compared to non-responders, whereas significant differences were found in trait anger and anger control based on treatment response. In contrast, Cahill and colleagues (2003) found significant changes in state anger co-occurring with changes in PTSD symptoms in a sample of women with chronic PTSD that received prolonged exposure therapy. A limitation to Cahill and colleagues' study was, however, that they did not compare changes in state and trait anger. Finally, Orth and Wieland (2006) conducted a meta-analysis to summarize the effect of PTSD treatment on anger expression. They found that PTSD treatment lead to reductions across anger expressions; but, the effect size was greater for internalized anger than externalized anger. Based on the findings of the aforementioned studies, the lack of treatment effect on anger in the current trial may result from the DAR-5 focusing on trait and externalized anger rather than state and internalized anger, which may be more amenable to changes in PTSD treatment.

Neither CPT nor SM had significant effects on mean total DTS scores or on the DTS Appraisal and Regulation subscales. Both conditions lead to improvement in scores on the Absorption subscale; but, SM had a greater effect (Cohen's $d=-0.99$ ) than CPT (Cohen's $d=0.59$ ), especially for SM participants who reported a single bullying victimization experience (Cohen's $d$ of 0.98). A large improvement was also found for participants in SM on the DTS Tolerance subscale (Cohen's $d=-1.03$ ). This effect was 
greater for participants in SM who endorsed experiencing a single victimization experience compared to numerous bullying victimization experiences (Cohen's $d=0.84$ ). Previous research suggests that interventions that focus on mind and body skills, such as relaxation and yoga, effectively increase distress tolerance (Kraemer, Luberto, O'Bryan, Mysinger, \& Cotton, 2016; Medina, Hopkins, Powers, Baird, \& Smits, 2015). However, cognitive behavioural therapies have also been found effective for increased distress tolerance (McHugh, Kertz, Weiss, Baskin-Sommers, Hearon, \& Björgvinsson, 2014), and improvements in distress tolerance predict treatment outcomes in trauma treatment, with higher ability to withstand distress associated with lower PTSD symptoms post-treatment (Banducci, Connolly, Vujanovic, Alvarez, \& Bonn-Miller, 2017). While the present findings that SM led to increased distress tolerance are in line with the research literature, the moderate effect of CPT on the DTS Absorption subscale and no effect on the total scale and other subscales contrast previous research. In line with the quadratic pattern found in the session-to-session changes in $\mathrm{CPT}$, it is possible that distress tolerance would have increased in the CPT condition if treatment was longer in duration.

Total scores on the IUS-12 and scores on the Inhibitory subscale were not associated with a group by time effect in either treatment group. Scores on the Prospective subscale increased over time in SM compared to the waitlist (Cohen's $d$ of 0.73); but, participants in SM who endorsed a single bullying victimization experience had greater reductions than participants who reported more than one bullying victimization experience (Cohen's $d$ of -0.68). No group by time interaction effects were found for CPT on the Prospective subscale. The lack of effect of CPT on IU in this trial is surprising given that CPT had a large effect on PTSD symptoms, and previous research 
suggests that reductions in PTSD symptoms are related to improvements in IU (Boswell, Thompson-Hollands, Farchione, \& Barlow, 2013). These findings could be related to the lack of treatment effect on symptoms of social anxiety. Correlation of IUS-12 score was higher in this trial with SIPS scores than PCL-5 scores. Session-by-session changes in SIPS and DASS-21 total scores indicated that symptoms of social anxiety increased over the course of CPT and that they only began to decline at the end of treatment. It is possible that scores on the IUS-12 would have followed a similar pattern, suggesting that CPT could have led to significant improvements in symptoms of anxiety and IU with more sessions.

The effects of CPT and SM on anxiety sensitivity differed across the ASI-3 total scale and subscales. Mean total scores on the ASI-3 increased over time in SM, compared to the waitlist (Cohen's $d=0.48$ ), but decreased on the ASI-3 Social concerns subscale (Cohen's $d \mathrm{~s}=-0.62$ to -0.82 ). SM had no effect on the Physical and Cognitive concerns subscales. Participants in CPT who reported a single bullying victimization experience had greater reductions in total mean ASI-3 scores over time compared to the waitlist and participants in CPT who reported more than one bullying victimization experience (Cohen's $d=-0.49$ ). CPT also had a large effect on the ASI-3 Physical concerns subscale scores for participants with a remote bullying victimization experience compared to participants with a recent bullying victimization experience (Cohen's $d=-0.95$ ). CPT had no effect on the Social and Cognitive concerns subscales. These findings are somewhat consistent with previous research, although significant effects were expected to be found across all subscales. Both cognitive behavioural therapies and relaxation techniques have been found effective for reducing anxiety sensitivity (Feinstein et al., 2018; Gutner et al., 
2013). The findings that CPT had an impact on the Physical concerns subscale whereas the SM did not are unexpected; indeed, CPT did not focus specifically on the physical aspects of anxious arousal whereas SM did. Gutner et al. (2013), however, also found that CPT led to reductions on the ASI-3 Physical concerns subscale.

Taken together, the results of this trial suggest that modified CPT internetdelivered over the course of approximately 12 weeks is effective for reducing symptoms of PTSD from bullying victimization, maladaptive self-appraisals when lifetime bullying victimization is not chronic (e.g., discrete exposure to bullying victimization in both primary school or later in life in the workplace), and concerns over the physical aspect of anxious arousal (i.e., ASI-3 Physical Concerns subscale) when bullying victimization experiences are not recent. Furthermore, CPT had small to moderate effects on negative other-beliefs, general symptoms of psychological distress (i.e., total scores on the DASS21), attentional absorption of distress (i.e., DTS Absorption subscale), and overall anxiety sensitivity. The results of this trial further suggest that internet-delivered SM over the course of approximately 12 weeks is effective for reducing general symptoms of psychological distress (i.e., total scores on DASS-21), attentional absorption of distress

(i.e., DTS Absorption subscale), and distress tolerance and that it is moderately effective in reducing negative other-beliefs related to bullying victimization.

\subsection{Summary of Secondary Measures}

Hypothesis 3-that level of resilience, perceived social support, and coping styles mediated the relationship between event centrality and strength of maladaptive appraisals—was partly supported. Event centrality did not have significant associations with resilience or perceived social support; but, there were significant relationships 
between event centrality and cognitive emotion regulation styles. The final regression, however, indicated that only catastrophizing mediated the relationship between event centrality and strength of maladaptive appraisals. These findings are in line with previous research indicating that individual differences in coping mediate the relationship between event centrality and maladaptive trauma appraisals (George, Park, \& Chaudoir, 2016). Catastrophizing is a cognitive emotion regulation style that involves explicitly focusing on the negative aspects of an experience. Catastrophizing predicts the development of PTSD following a traumatic event (Bryant \& Guthrie, 2016; Jenness et al., 2016) and is associated with increased emotional distress and anxiety after bullying victimization (Garnefski, Kraaij, \& Spinhoven, 2001; Garnefski \& Kraaij, 2014). Event centrality, which refers to the extent to which a traumatic experience has become central to one's identify and perception of self, has been linked to both increased distress following a trauma and to posttraumatic growth. The results of this trial suggest that perceiving a bullying victimization experience as central to personal identity and understanding of the world becomes detrimental when there is a tendency to engage in catastrophizing (Boals \& Schuttler, 2011; Schuttler \& Boals, 2011). Given that social support has been identified as an important factor in mitigating distress associated with trauma and bullying victimization, the current finding that social support was not a significant mediator in the relationship between event centrality and maladaptive appraisals is surprising (Cook et al., 2010; Ozer et al., 2008).

Hypothesis 4-that number of bullying victimization experiences would moderate the impact of maladaptive appraisals on psychological distress (i.e., symptoms of social anxiety and PTSD, general distress, and anger)—was partly supported. Significant 
associations were found between maladaptive appraisals, symptoms of social anxiety and PTSD, general distress, and anger, with greater strength in appraisals leading to more psychological distress. Number of bullying victimization experiences was, however, only a moderator in the relationship between maladaptive appraisals and anger. Greater anger was associated with stronger maladaptive appraisals when participants endorsed more than one bullying victimization experience. These findings are in line with previous research indicating that chronic bullying victimization is associated with greater psychological distress when compared to bullying that is confided to a single time or context (Ladd \& Ladd, 2001; Smith et al., 2003).

Taken together, the results of the testing of secondary measures suggests that higher event centrality is maladaptive when cognitive emotion regulation involves catastrophizing because the combined effect of these constructs leads to stronger negative appraisals about oneself and the world. Furthermore, anger arising from maladaptive appraisals of bullying victimization experiences is greater when individuals have been subjected to more than one bullying victimization experience.

\subsection{Implications}

The main purpose of this trial was to examine the effects of a new treatment tailored to resolve the negative psychological impact of bullying victimization using rigorous testing of the outcomes against inactive and active control conditions. Bullying victimization is associated with numerous psychological concerns, including depression and anxiety; but, given that the onset of problems has been linked directly to the bullying experiences for many victims (Takizawa et al., 2014), it stands to reason that this experience should be the target of treatment. The similarities between bullying 
victimization and trauma further support focusing treatment for victims on the memories and appraisals of the bullying experience. Specifically, PTSD is highly comorbid with other psychological concerns and, although evidence-based treatments center around the memory and interpretation of the trauma, simultaneous remissions frequently occur in symptoms of comorbid conditions (Resick et al., 2014; van Minnen, Zoellner, Harned, \& Mills, 2015). Establishing an effective and evidence-based therapy to treat the distress associated with bullying victimization is imperative, considering the high prevalence of bullying in schools and workplaces, across age groups and cultures, and the pervasive negative effects of victimization. The modified, internet-delivered CPT protocol evaluated in this trial is a promising avenue for treatment of bullying victimization that is effective for symptoms of PTSD, convenient and affordable, and well perceived by adults self-identified as victims of bullying.

The results of this trial further suggest important variables in the pathways from bullying victimization to maladjustment. Not all victims of bullying experience long-term negative outcomes, which suggests there exist important individual differences in susceptibility to the detrimental effects of victimization. By identifying individual risk and resiliency factors, focus of treatment for the psychological impact of bullying victimization may be refined and effective school and workplace interventions may be developed to mitigate the detrimental effects of victimization. This trial examined trauma appraisals in combination with various individual and social factors that were proposed to contribute to the maladjustment following bullying victimization. Higher perceived event centrality of bullying victimization, in combination with a tendency to engage in catastrophizing to regulate emotions, was associated with stronger maladaptive appraisals. 
This finding suggests that screening for a tendency to engage in catastrophizing could be important for targeting this unhelpful cognitive emotion regulation strategy in treatment for victims of bullying. The findings of this trial further suggest that maladaptive appraisals lead to more anger when exposure to bullying victimization is chronic. These findings imply that intervention for victims of chronic bullying victimization may benefit from a specific emphasis on anger.

\subsection{Limitations}

Limitations of the trial include that participants self-identified as victims of bullying and bullying victimization was assessed retrospectively. Long time may have passed since participants were exposed to bullying victimization, and memories from various other stressful life events may have interfered with recollection of the impact of the experience. All participants in the sample also reported life-time exposure to trauma; but, the specific timing of the traumatic experience was not assessed in this trial. Other traumatic experiences may, thus, have exacerbated or even been the primary source of the distress that participants attributed to their bullying victimization experiences. The majority of participants endorsed a remote experience of bullying victimization; but, amongst participants who indicated a recent bullying victimization experience, several reported that the bullying was ongoing in their workplace. Current bullying victimization was not an exclusion criteria in the trial, which may have impacted the findings, as treatment which focuses on cognitive trauma appraisal while an individual is exposed to ongoing trauma may not be as effective as treatment that is conducted once the trauma has passed (e.g., Taylor, 2017). Indeed, differences were found between participants who reported remote and recent trauma on many of the outcome measures. 
Another limitation of this trial is that the assessment of psychological distress did not include clinical diagnosis. The majority of participants reported clinically significant symptoms of distress, but they were not asked to indicate their primary concern. However, the aim of the trial was to examine whether internet-delivered therapist-guided CPT led to reductions of distress associated with bullying victimization rather than specific changes in diagnostic status. The trial also did not include evaluation of characterological features and symptoms of personality disorders, which, if present, could have impeded the effectiveness of treatment, especially with regards to general distress and distress tolerance (Hembree, Cahill, \& Foa, 2004).

There are also few limitations to the design of the trial. Specifically, neither participants nor the principal investigator, who also served as the therapist, were blind to treatment group allocation. Randomization into group reduced bias at the outset of trial, but the estimation of treatment outcomes might have been biased by participants' perception of their allocated treatment as well as possible differential researcher behaviour. However, the benefits of the same therapist treating all participants may have reduced bias introduced by different therapist behaviour. The trial utilized intent-to-treat analysis which has both pros and cons. Specifically, intent-to treat analysis preserves the sample size by including participants that did not comply or dropped out of treatment and gives an unbiased estimate of the treatment effect, but it also increases the risk of type II error due to the conservative estimation of treatment effect (Gupta, 2011). The sample in the current trial also consistent predominately of Caucasian women, employed full time, married, and at the higher end of socioeconomic status, which limits the generalizability of the results. A large number of individuals residing in other Canadian provinces than 
Saskatchewan, and in one case abroad, were interested in receiving services through participation in this trial for psychological distress associated with bullying victimization. Given the restrictions imposed on the provision of psychological services by licensure and regulatory bodies in Canada, however, these individuals were not eligible to participate in the trial, which further limits the generalizability of the findings of this trial. Finally, the sample also likely presented with a restricted range of risk and resilience factors; indeed, to be eligible to participate in the trial, individuals had to endorse significant distress associated with the bullying victimization experiences. Since the distress from the bullying victimization persisted, it is likely that individuals in this sample reported more risk factors and less resilience factors (e.g., limited social support) than victims who recover from the bullying.

\subsection{Future Directions}

This trial is the first to test the effectiveness of a modified version of CPT for maladaptive outcomes associated with bullying victimization. Further research is necessary for continued development and refinement of the treatment in terms of duration and module content to establish that it is effective. The trial should also be replicated using a larger and more diverse sample, and with a longer follow-up assessment, and possible more flexible timelines for completing modules and terminating therapy to reduce drop-out (Hadjistavropoulos et al., 2018). Timing of bullying victimization experiences should be differentiated further than was done in the current trial and assessment of other stressful and traumatic life experiences than bullying victimization should be more time specific. Since bullying victimization can lead to pervasive longterm outcomes that persist from youth to adulthood, adapting the internet-delivered CPT 
protocol used in this trial to children and youth exposed to bullying victimization would also be an important avenue for future research.

The findings that SM outperformed CPT on some of the symptom measures, including general psychological distress and distress tolerance, suggests a possible avenue for future research involving combining the two interventions into a more comprehensive or a longer treatment; but stress management skills, such as controlled breathing, are commonly included in PTSD treatment (e.g., prolonged exposure therapy, Taylor et al., 2003; Taylor, 2007). Preliminary research evidence also exists on the effectiveness of incorporating distress tolerance and emotion regulation skills to traumafocused treatment for adults with a history of childhood sexual abuse; specifically, treatment is delivered in stages over 20 sessions, beginning with group intervention that aims at increasing distress tolerance and emotion regulation skills, and psychoeducation, followed by individual exposure-based intervention and cognitive restructuring (Cloitre, Koenen, Cohen, \& Han, 2002; House, 2006). The rational for the modification of traumafocused treatment for adults with a history of childhood abuse is that such history is frequently associated with greater difficulty regulating emotions and tolerating distress, compared to adult exposure to abuse (Cloitre, Miranda, Stovall-McClough, \& Han, 2005). Based on the parallels found in neurobiological and psychological research between childhood abuse and bullying victimization early in life (Vaillancourt, Hymel, \& McDougall, 2013), a longer course of trauma-focused treatment that incorporates stress management and distress tolerance skills may be beneficial.

A different way of understanding the psychological distress associated with adult bullying victimization comes from research on a distinct type of adjustment-related 
difficulty characterized by embitterment. Linden (2003) coined the term posttraumatic embitterment disorder (PTED) in reference to feelings of resentment or bitterness that are frequently accompanied by vengefulness and helplessness, and develop following a significant negative life event, such as conflict at work or divorce. The negative life event is different from trauma in that there is no exposure to actual or threatened death, serious injury, or sexual violence but instead, feelings of unjustness and humiliation (Linden, Baumann, Rotter, \& Schippan, 2007). Similar to PTSD, PTED is associated with symptoms of recurrent memories, avoidance to external reminders of the event, arousal to external memory cues, and negative alterations in mood and cognition (e.g., blaming oneself for the negative event, dysphoria, aggression, reduced motivation; Linden, 2003). Symptoms of PTED differ from PTSD in that thoughts and memories about the negative life event are not avoided to the same extent as internal reminders of trauma, and the affective disturbance is less pervasive than in PTSD (Linden et al., 2007). The criteria for PTED further specify that the symptoms must last for at least six months and cause significant distress and functional impairment, and may not be better explained by a different diagnosis (Linden, 2003, Linden et al., 2007, Linden, Baumann, Rotter, \& Schippan, 2009). Karatuna and Gök (2014) found evidence of an association between PTED and exposure to workplace bullying. They used anonymous survey data from 397 adults, and found that individuals subjected to bullying victimization at work had significantly more symptoms of PTED compared to adults that had not been bullied. Characterizing psychological distress associated with bullying victimization as PTED had implications for treatment. Specifically, Linden, Baumann, Lieberei, Lorenz, and Rotter (2011) compared the effects of routine transdiagnostic CBT to CBT combined with 
elements of wisdom therapy (i.e., therapy strategies that focus on coping with unsolvable life problems) in an inpatient sample. The combined CBT treatment outperformed the routine transdiagnostic $\mathrm{CBT}$ in reducing PTED, suggesting that a specific emphasis on wisdom skills (e.g., acceptance of emotions, empathy) is needed in treatment for PTED. Recently, Michailidis and Cropley (2019) examined the effectiveness of expressive writing compared to factual writing of daily activities, in a randomized controlled trial in a sample of working adults that had been exposed to a negative social event and reported embitterment. Embitterment decreased over the course of three months in both groups. The authors suggested that both writing conditions were effective because they offered a distraction from embitterment-related distress and cognitions; but, they emphasized that more research is needed on effective treatment. Directions for future research in this area include disentangling embitterment further from symptoms of posttraumatic stress. Embitterment might, for example, more appropriately capture the psychological distress associated with workplace bullying, whereas posttraumatic stress could encompass the long-standing psychological impact of bullying in youth. Attribution of blame could be especially important for differentiating between embitterment and posttraumatic stress from bullying victimization; specifically, embitterment might be associated to a greater extent to other-blame while posttraumatic stress could relate more to self-blame.

\subsection{Conclusions}

Bullying is a common problem that has significant negative impact on the victim but bullying interventions are frequently not implemented in schools and workplaces. Previous research indicates that individuals exposed to bullying victimization frequently blame themselves, and the availability of effective interventions that focus on such 
maladaptive appraisals and alleviation of associated secondary emotions (e.g., shame) and other psychological distress is, therefore, imperative. The modified CPT protocol tested in this trial is a promising treatment for alleviating the psychological distress associated with bullying victimization. 


\section{REFERENCES}

Aderka, I.M., Foa, E.B., Applebaum, E., Shafran, N., \& Gilboa-Schectman, E. (2011). Direction of influence between posttraumatic and depressive symptoms during prolonged exposure therapy among children and adolescents. Journal of Consulting and Clinical Psychology,79, 421- 425. doi:10.1037/a0023318

Alfonsson, S., Olsson, E. M.G, \& Hursti, T. (2016). Motivation and treatment credibility predicts dropout, treatment adherence, and clinical outcomes in an internetbased cognitive behavioural relaxation program: A randomized controlled trial. Journal of Medical Internet Research, 18, e52. doi:10.2196/jmir.5352

Alfonsson, S., Olsson, E. M. G., Linderman, S., Winnerhed, S., \& Hursti, T. (2016). Is online treatment adherence affected by presentation and therapist support? A randomized controlled trial. Computers in Human Behavior, 60, 550-558. doi:10.1016/j.chb.2016.01.035

Allan, N. P., Raines, A . M., Capron, D. W., Norr, A. M., Zvolensky, M. J., \& Schmidt, N. B. (2014). Identification of anxiety sensitivity classes and clinical cut-scores in a sample of adult smokers: Results from a factor mixture model. Journal of Anxiety Disorders, 28, 696 - 703. doi:10.1016/j.janxdis.2014.07.006

Allanson, P. B., Lester, R. R., \& Notar, C. E. (2015). A history of bullying. International Journal of Education and Social Science, 2(12), 31-36.

Anderson, D. (2012). Hierarchical Linear Modeling (HLM): An introduction to key concepts within cross-sectional and growth modeling frameworks. (Report No. 1308). Behavioral Research and Teaching: Eugene, Oregon. 
Andersson, G., Rozental, A., Shafran, R., \& Carlbring, P. (2018). Long-term effects of internet-supported cognitive behaviour therapy. Expert Review of Neurotherapeutics, 18, 21-28. doi:10.1080/14737175.2018.1400381

American Psychiatric Association. (2013). Diagnostic and statistical manual of mental disorders (5th ed.). Washington, DC: Author.

Antoniadou, N., \& Kokkinos, C. M. (2015). Cyber and school bullying: Same or different phenomena? Aggression and Violent Behavior, 25, 363-372. doi:10.1016/j.avb.2015.09.013

Asmundson, G. J.G, \& Stapleton, J. A. (2008). Associations between dimensions of anxiety sensitivity and PTSD symptom clusters in active-duty police officers. Cognitive Behaviour Therapy, 37, 66-75. doi:10.1080/16506070801969005

Asmundson, G. J.G., Thorisdottir, A.S., Roden-Foreman, J.W., Baird, S.O., Witcraft, S.M., Stein, A.T., Smits, J.A.J., \& Powers, M.B. (2019). A meta-analytic review of cognitive processing therapy for adults with posttraumatic stress disorder. Cognitive Behaviour Therapy, 48, 1-14. doi:10.1080/16506073.2018.1522371.

Banducci, A. N., Connolly, K. M., Vujanovic, A.A., Alvarez J., \& Bonn-Miller, M. O. (2017). The impact of changes in distress tolerance on PTSD symptom severity post-treatment among veterans in residential trauma treatment. Journal of Anxiety Disorders, 47, 99-105. doi:10.1016/j.janxdis.2017.01.004

Banerjee, S., \& Severn, M. (2018). e-Therapy interventions for the treatment of posttraumatic stress disorder: A review of clinical evidence. CADTH rapid response report: Peer-reviewed summary with critical appraisal: Ottawa. Retrieved from: 
https://www.cadth.ca/sites/default/files/pdf/htis/2018/RC0985\%20etherapy\%20for\%20PTSD\%20Final.pdf

Baird, C. L., \& Sands, L. (2004). A pilot study of the effectiveness of guided imagery with progressive muscle relaxation to reduce chronic pain and mobility difficulites of osteoarthritis. Pain Management Nursing, 5, 97-104. doi:10.1016/j.pmn.2004.01.003

Baldry, A. C., \& Farrington, D. P. (2005). Protective factors as moderators of risk factors in adolescence bullying. Social Psychology of Education, 8, 263-284. doi:10.1007/s11218-005-5866-5

Barlow, D. H. (2000). Unraveling the mysteries of anxiety and its disorders from the perspective of emotion theory. American Psychologist, 55, 1247-1263. doi:10.1037/0003-066X.55.11.1247

Bauer, S., Lambert, M. J., \& Nielsen, S. L. (2004). Clinical significance methods: A comparison of statistical techniques. Journal of Personality Assessment, 82, 6070. doi:10.1207/s15327752jpa8201_11

Beck, J. G., Coffey, S. F., Palyo, S. A., Gudmundsdottir, B., Miller, L. M. \& Colder, C. R. (2004). Psychometric properties of the posttraumatic cognitions inventory (PTCI): a replication with motor vehicle accident survivors. Psychological Assessment, 16, 289-298. doi:10.1037/1040-3590.16.3.289

Berger, K.S. (2007). Update on bullying at school: Science forgotten? Developmental Review, 27, 90-126. doi:10.1016/j.dr.2006.08.002

Berenz, E. C., Vujanovic, A. A., Coffey, S. F., \& Zvolensky, M. J. (2012). Anxiety sensitivity and breath-holding duration in relation to PTSD symptom severity 
among trauma exposed adults. Journal of Anxiety Disorders, 26, 134-139. doi:10.1016/j.janxdis.2011.10.004

Berntsen, D., \& Rubin, D. C. (2006). The centrality of event scale: A measure of integrating a trauma into one's identity and its relation to post-traumatic stress disorder symptoms. Behaviour Research and Therapy, 44, 219-231. doi:10.1016/j.brat.2005.01.009

Blevins, C.A., Weathers, F. W., Davis, M. T., Witte, T. K. \& Domino, J. L. (2015). The posttraumatic stress disorder checklist for DSM-5 (PCL-5): Development and initial psychometric evaluation. Journal of Traumatic Stress, 28, 489 - 498. doi:10.1002/jts.22059

Blood, G. W., \& Blood, I. M. (2016). Long-term consequences of childhood bullying in adults who stutter: Social anxiety, fear of negative evaluation, self-esteem, and satisfaction with life. Journal of Fluency Disorders. doi:10.1016/j.jfludis.2016.10.002

Boals, A., \& Schuettler, D. (2011). A double-edged sword: Event centrality, PTSD and posttraumatic growth. Applied Cognitive Psychology, 25, 817-822. doi:10.1002/acp.1753

Boswell, J. F., Thompson-Hollands, J., Farchione, T. J., \& Barlow, D. H. (2013). Intolerance of uncertainty: A common factor in the treatment of emotional disorders. Journal of Clinical Psychology, 69. doi:10.1002/jclp.21965. doi:10.1002/jclp.21965

Boulton, M. J., Smith, P. K., \& Cowie, H. (2010). Short-term longitudinal relationships between children's peer victimization/bullying experiences and self-perceptions: 
Evidence for reciprocity. School Psychology International, 31, 296-311. doi:10.1177/0143034310362329

Bowes, L., Maughan, B., Caspi, A., Moffitt, T. E., Arseneault, L. (2010). Families promote emotional and behavioural resilience to bullying: Evidence of an environmental effect. Journal of Child Psychology and Psychiatry, 51, 809-817. doi:10.1111/j.1469-7610.2010.02216.x

Bremner, J. D., \& Vermetten, E. (2001). Stress and development: Behavioral and biological consequences. Development and Psychopathology, 13, 473-489. doi:10.1017/s0954579401003042

Brown, R. A., Lejuez, C. W., Strong, D. R., Kahler, C. W., Zvolensky, M. J., Carpenter, L. L., ... \& Price, L. H. (2009). A prospective examination of distress tolerance and early smoking lapse in adult self-quitters. Nicotine and Tobacco Research,11, 493-502. doi:10.1093/ntr/ntp041

Bryant, R. A., \& Guthrie, R. M. (2005). Maladaptive appraisals as a risk factor for posttraumatic stress: a study of trainee firefighters. Psychological Science, 16, 749-752. doi:10.1111/j.1467-9280.2005.01608.x

Cahill, S. P., Rauch, S.A., Hembree, E.A., \& Foa, E.B. (2003). Effect of cognitivebehavioural treatments for PTSD on anger. Journal of Cognitive Psychotherapy, 17, 113-131. doi:10.1891/jcop.17.2.113.57434

Carlbring, P., Ekselius, L., Andersson, G. (2003). Treatment of panic disorder via the internet: A randomized trial of CBT vs. applied relaxation. Journal of Behavior Therapy and Experimental Psychiatry, 34, 129-140. doi:10.1016/S00057916(03)00026-0 
Carleton, R. N., Norton, M. A. P. J., \& Asmundson, G. J. G. (2007). Fearing the unknown: A short version of the Intolerance of Uncertainty Scale. Journal of Anxiety Disorders, 21, 105-117. doi:10.1016/j.janxdis.2006.03.014

Carleton, R. N., Collimore, K. C., Asmundson, G. J. G., McCabe, R. E., Rowa, K., \& Antony, M. M. (2009). Refining and validating the social interaction anxiety scale and the social phobia scale. Depression and Anxiety, 26, 71-81. doi:10.1002/da.20480

Carleton, R. N., Peluso, D. L., Collimore, K. C., \& Asmundson, G. J. G. (2011). Social anxiety and posttraumatic stress symptoms: The impact of distressing social events. Journal of Anxiety Disorders, 25, 46-57.

doi:10.1016/j.janxdis.2010.08.002

Carney, A.M. \& Merell, K.W. (2001). Bullying in schools: Perspectives on understanding and preventing an international problem. School Psychology International, 22, 364-382. doi:10.1177/0143034301223011

Cassidy, T., McLaughlin, M., \& McDowell, E. (2014). Bullying and health at work: the mediating roles of psychological capital and social support. Work \& Stress, 28, 255-269. doi:10.1080/02678373.2014.927020

Chard, K. M. (2005). An evaluation of cognitive processing therapy for the treatment of posttraumatic stress disorder related to childhood sexual abuse. Journal of Consulting and Clinical Psychology, 73, 965-971. doi:10.1037/0022006X.73.5.965

Chard, K. M., Ricksecker, E.G., Healy, E.T., Karlin, B.E., \& Resick, P.A. (2012). Dissemination and experience with cognitive processing therapy. Journal of 
Rehabilitation Research and Development, 49, 667-678. doi:10.1682/JRRD.2011.10.0198

Chen, Z., Williams, K. D., Fitness, J., \& Newton, N. C. (2008). When hurt will not heal: Exploring the capacity to relive social and physical pain. Psychological Science, 19, 789-795. doi:10.1111/j.1467-9280.2008.02158.x

Clara, I. P., Cox, B. J., Enns, M. W., Murray, L. T., \& Torgrudc, L. J. (2003). Confirmatory factor analysis of the multidimensional scale of perceived social support in clinically distressed and student samples. Journal of Personality Assessment, 81, 265-270. doi:10.1207/S15327752JPA8103_09

Clark, D. M. (2001). A cognitive perspective on social phobia. In W. R. Crozier \& Le. E., Alden (Eds.). The international handbook of social anxiety: concepts, research and interventions relating to the self and shyness. New York: John Wiley \& Sons Ltd.

Cloitre, M., Koenen, K. C., Cohen, L. R., \& Han, H. (2002). Skills training in affective and interpersonal regulation followed by exposure: a phase-based treatment for PTSD related to childhood abuse. Journal of Consulting \& Clinical Psychology, 70, 1067-1074. doi:10.1037/0022-006x.70.5.1067

Cloitre, M., Miranda, R., Stovall-McClough, K. C., \& Han, H. (2005). Beyond PTSD: emotion regulation and interpersonal problems as predictors of functional impairment in survivors of childhood abuse. Behavior Therapy, 36,119-124. doi:10.1016/s0005-7894(05)80060-7 
Cook, C. R., Williams, K. R., Guerra, N. G., Kim, T. E., \& Sadek, S. (2010). Predictors of bullying and victimization in childhood and adolescence: A meta-analytic investigation. School Psychology Quarterly, 25, 65-83. doi:10.1037/a0020149

Copeland, W. E., Wolke, D., Angold, A., \& Costello, E. J. (2013). Adult psychiatric outcomes of bullying and being bullied by peers in childhood and adolescence. JAMA Psychiatry, 70, 419-426. doi:10.1001/jamapsychiatry.2013.504

Deacon, B., \& Abramowitz, J. S. (2006). Anxiety sensitivity and its dimensions across the anxiety disorders. Journal of Anxiety Disorders, 20, 837-857. doi:10.1016/j.janxdis.2006.01.003

Deffenbacher, J. L., Oetting, E.R., Thwaites, G.A., Lynch, R.S., Baker, D.A., Stark, R.S., Thacker, S., Eiswerth-Cox, L. (1996). State-trait anger theory and the utility of the trait anger scale. Journal of Counseling Psychology, 43, 131-148. doi:10.1037//0022-0167.43.2.131

Due, P., Holstein, B. E., Lynch, J., Diderichsen, F., Gabhain, S. N., Scheidt, P., \& Currie, C. (2005). Bullying and symptoms among school-aged children: International comparative cross sectional study in 28 countries. The European Journal of Public Health, 128-132. doi:10.1093/eurpub/cki105

Edmonds, M., Hadjistavropoulos, H., Schneider, L. H., Dear, B. F., \& Titov, N. (2018). Who benefits most from therapist-assisted internet-delivered cognitive behavior therapy in clinical practice? Predictors of symptom change and dropout. Journal of Anxiety Disorders, 54, 24 - 32. doi:10.1016/j.janxdis.2018.01.003 
Efird, J. (2011). Blocked randomization with randomly selected block sizes. International Journal of Environmental Research and Public Health, 8, 15-20. doi:10.3390/ijerph8010015

Ehlers, A. \& Clark, D.M. (2000). A cognitive model of posttraumatic stress disorder. Behavior Research and Therapy, 38, 319-345. doi:10.1016/s00057967(99)00123-0

Einarsen, S., \& Skogstad, A. (1996). Bullying at work: Epidemiological findings in public and private organizations. European Journal of Work and Organizational Psychology, 5, 185-201. doi:10.1080/13594329608414854

Einarsen, S., Hoel, H., Zapf, D., \& Cooper, C. (2011). The concept of bullying and harassment at work: the European tradition. In, Bullying and Harassment in the Workplace: Developments in Theory, Research and Practice ( $2^{\text {nd }}$ ed.). Taylor \& Francis: Boca Raton, FL, USA.

Erwin, B. A., Heimberg, H. G., Marx, B. P., \& Franklin, M. E. (2006). Traumatic and socially stressful life events among persons with social anxiety disorder. Journal of Anxiety Disorders, 20, 896-914. doi:10.1016/j.janxdis.2005.05.006

Eslea, M., \& Rees, J. (2001). At what age are children most likely to be bullied at school? Aggressive Behavior, 27, 419-429. doi:10.1002/ab.1027

Espelage, D. L., \& Swearer, S. M. (2003). Research on school bullying and victimization: what have we learned and where do we go from here? School Psychology Review, 12(1), 365-383.

Farrington, D. P., \& Ttofi, M. M. (2009). School-Based Programs to Reduce Bullying and Victimization. Campbell Systematic Reviews. doi:610.4073/csr.2009.6 
Feinstein, J.S., Khalsa, S.S., Yeh, H., Zoubi, O.A., Arevian, A.C., Wohlrab, C., ...\& Paulus, M.P. (2018). The elicitation of relaxation and interoceptive awareness using floatation therapy in individuals with high anxiety sensitivity. Biological Psychiatry,3, 555-562. doi:10.1016/j.bpsc.2018.02.005

Feldman, G., Greeson, J., \& Senville, J. (2010). Differential effects of mindful breathing, progressive muscle relaxation, and loving-kindness meditation on decentering and negative reactions to repetitive thoughts. Behaviour Research and Therapy, 48, 1002-1011. doi: 10.1016/j.brat.2010.06.006

Fleming, J., \& Ledogar, R. J. (2008). Resilience, an evolving concept: a review of literature relevant to aboriginal research. Pimatisiwin, 6, 7-23. Retrieved from https://www.ncbi.nlm.nih.gov/pmc/articles/PMC2956753/pdf/nihms387.pdf

Foa, E. B., Ehlers, A., Clark, D.M., Tolin, D. F. \& Orsillo, S. M. (1999). The posttraumatic cognitions inventory (PTCI): Development and validation. Psychological Assessment, 11, 303-314. doi:10.1037/t00499-000

Forbes, D., Hawthorne, G., Elliott, P., Tony, M., Biddle, D., Creamer, M., Novaco, R. W. (2004). A concise measure of anger in combat-related posttraumatic stress disorder. Journal of Traumatic Stress, 17, 249-256. doi:10.1023/b:jots.0000029268.22161.bd

Forbes, D., Lloyd, D., Nixon, R.D.V., Elliott, P., Varker, T., Perry, D., ...Creamer, M. (2012). A multisite randomized controlled effectiveness trial of cognitive processing therapy for military-related posttraumatic stress disorder. Journal of Anxiety Disorders, 26, 442-452. doi: 10.1016/j.janxdis.2012.01.006 
Forbes, D., Alkemade, N., Hopcraft, D., Hawthorne, G., O’Halloran, P., Elhai, J. D. ... Lewis, V. (2014). Evaluation of the Dimensions of Anger Reactions-5 (DAR-5) Scale in combat veterans with posttraumatic stress disorder. Journal of Anxiety Disorders, 28, 830-835. doi:10.1016/j.janxdis.2014.09.015

Forbes, D., Alkemade, N., Mithcell D., Elhai, J. D., McHugh, T., Bates, G., ... Lewis, V. (2014). Utility of the Dimensions of Anger Reactions-5 (DAR-5) Scale as a brief anger measure. Depression and Anxiety, 31, 166-173. doi:10.1002/da.22148

Gallagher, M. W., \& Resick, P. A. (2012). Mechanisms of change in cognitive processing therapy and prolonged exposure therapy for PTSD: Preliminary evidence for the differential effects of hopelessness and habituation. Cognitive Therapy and Research, 36, 750-755. doi: 10.1007/s10608-011-9423-6

Galovski, T. E., Blain, L. M., Mott, J. M., Elwood, L., \& Houle, T. (2012). Manualized therapy for PTSD: Flexing the structure of cognitive processing therapy. Journal of Consulting and Clinical Psychology, 80, 968-981. doi: $10.1037 / \mathrm{a} 0030600$

Galovski, T.E., Elwood, L.S., Blain, L.M., \& Resick, P.A. (2014). Changes in anger in relationship to responsivity to PTSD treatment. Psychological Trauma, 6, 56-64. doi:10.1037/a0031364.

Galovski, T.E., Harik, J. M., Blain, L. M., Farmer, C., Turner, D., \& Houle, T. (2016). Identifying patterns and predictors of PTSD and depressive symptom change during cognitive processing therapy. Cognitive Therapy and Research, 40, 617626. doi:10.1007/s10608-016-9770-4 
Gardner, B., Rose, J., Mason, O., Tyler, P., Cushway, D. (2005). Cognitive therapy and behavioural coping the management of work-related stress: an intervention study. Work and Stress, 19, 137-152. doi:10.1080/02678370500157346

Garnefski, N., \& Kraaij, V. (2014). Bully victimization and emotional problems in adolescents: Moderation by specific cognitive coping strategies? Journal of Adolescence, 37, 1153-1160. doi:10.1016/j.adolescence.2014.07.005

George, L.S., Park, C. L., \& Chaudoir, S. R. (2016). Examining the relationship between trauma centrality and posttraumatic stress disorder symptoms: a moderated mediation approach. Traumatology, 22, 85-93. doi:10.1037/trm0000063

Gibb, S. J., Horwood, J. \& Fergusson, D. M. (2011). Bullying victimization/perpetration in childhood and later adjustment: Findings from a 30 year longitudinal study. Journal of Aggression, Conflict and Peace Research, 3, 82-88. doi:10.1108/17596591111132891

Graham, S. (2016). Victims of bullying in schools. Theory into practice, 55, 136-144. doi:10.1080/00405841.2016.1148988

Groleau, J. M., Calhoun, L. G., Cann, A., \& Tedeschi, R. G. (2013). The role of centrality of events in posttraumatic distress and posttraumatic growth. Psychological Trauma: Theory, Research, Practice, and Policy, 5, 477-483. doi:10.1037/a0028809

Gupta, S. K. (2011). Intention-to-treat concept: A review. Perspectives in Clinical Research, 2, 109-112. doi:10.4103/2229-3485.83221

Gutner, C. A., Nillni, Y. I., Suvak, M., Wiltsey-Stirman, S., \& Resick, P. A. (2013). Longitudinal course of anxiety sensitivity and PTSD symptoms in cognitive- 
behavioral therapies for PTSD. Journal of Anxiety Disorders, 27, 728-734. doi:10.1016/j.janxdis.2013.09.010

Hadjistavropoulos, H. D., Pugh, N. E., Hesser, H., \& Andersson, G. (2016). Therapeutic alliance in internet-delivered cognitive behaviour therapy for depression or generalized anxiety. Clinical Psychology \& Psychotherapy, 24, 451- 461. doi:10.1002/cpp.2014

Hadjistavropoulos, H. D., Faller, N. Y., Klatt, A., Nugent, M. N., Dear, B. F., \& Titov, N. (2018). Patient perspectives on strengths and challenges of therapist-assisted internet-delivered cognitive behaviour therapy: Using the patient voice to improve care. Community Mental Health Journal, 57, 944-950.

doi:10.1007/s10597-018-0286-0

Hackmann, A., Clark, D. M., \& McManus, F. (2000). Recurrent images in early memories in social phobia. Behaviour Research and Therapy, 38, 601-610. doi:10.1016/S0005

Hayes, S.A., Hope, D. A., VanDyke, M.M., \& Heimberg, R. G. (2017). Working alliance for clients with social anxiety disorder: relationship with session helpfulness and within-session habituation. Cognitive Behavioral Therapy, 36, 34-42. doi:10.1080/16506070600947624

Hawkins, L., Pepler, D. J., \& Craig, W. M. (2001). Naturalistic observations of peer interventions in bullying. Social Development, 10, 512-527. doi: 10.1111/14679507.00178

Hedman, E., Axelsson, E., Görling, A., Ritzman, C., Ronnheden, M., Alaoui, S. E. ....Ljótsson, B. (2014). Internet-delivered exposure-based cognitive- 
behavioural therapy and behavioural stress management for severe health anxiety: Randomized controlled trial. The British Journal of Psychiatry, 205, 307-314. doi:10.1192/bjp.bp.113.140913

Heimberg, R. G. (2002). Cognitive-behavioural therapy for social anxiety disorder: current status and future directions. Society of Biological Psychiatry, 51, 101108. doi:10.1016/s0006-3223(01)01183-0

Hejdenberg, J., \& Andrews, B. (2011). The relationship between shame and different types of anger: a theory-based investigation. Personality and Individual Differences, 50, 1278-1282. doi:10.1016/j.paid.2011.02.024

Hembree, E.A., Cahill, S. P., \& Foa, E.B. (2004). Impact of personality disorders on treatment outcome for female assault survivors with chronic posttraumatic stress disorder. Journal of Personality Disorders, 18, 117-127. doi:10.1521/pedi.18.1.117.32767

Hempill, S. A., Tollit, M., \& Herrenkohl, T. I. (2014). Protective factors against the impact of school bullying perpetration and victimization on young adult externalizing and internalizing problems. Journal of School Violence, 13, 125145. doi:10.1080/15388220.2013.844072

Henry, J. D., \& Crawford, J. R. (2005). The short-form version of the Depression Anxiety Stress Scales (DASS-21): Construct validity and normative data in a large nonclinical sample. British Journal of Clinical Psychology, 44, 227-239. doi:10.1348/014466505X29657

Hertz, M. F., Jones, S. E., Barrios, L., David-Ferdon, C., \& Holt, M. (2015). Association between bullying victimization and health risk behaviors among high school 
students in the United States. Journal of School Health, 85, 833-842.

doi:10.1111/josh.12339

Hodges, E. V. E., Boivin, M., Vitaro, F., \& Bukowski, W. M. (1999). The power of friendship: Protection against an escalating cycle of peer victimization. Developmental Psychology, 35, 94-101. http://dx.doi.org/10.1037/00121649.35.1.94

Hoertel, N., Le Strat, Y., Lavaud, P. \& Limosin, F. (2012). Gender effects in bullying: Results from a national sample. Psychiatry Research, 200, 921-927. doi:10.1016/j.psychres.2012.03.036

Hofmann, S.G. (2004). Cognitive mediation of treatment change in social phobia. Journal of Consulting and Clinical Psychology, 72, 393-399. doi:10.1037/0022006x.72.3.392

House, A. S. (2006). Increasing the usability of cognitive processing therapy for survivors of child sexual abuse. Journal of Child Sexual Abuse, 15, 87-103. doi:10.1300/j070v15n01_05

Howard, A. M., Landau, S., \& Pryor, J. B. (2014). Peer bystanders to bullying: Who wants to play with the victim? Journal of Abnormal Child Psychology, 42, 265276. doi:10.1007/s10802-013-9770-8

Hyman, S. M., Gold, S. N., Cott, M. A. (2003). Forms of social support that moderate PTSD in childhood sexual abuse survivors. Journal of Family Violence, 18, 295-300. doi: 10.1023/A:1025117311660 
Imel, Z. E., Laska, K., Jakupcak, M., \& Simpson, T. L. (2015). Meta-analysis of dropout in treatments for posttraumatic stress disorder. Journal of Consulting and Clinical Psychology, 81, 394-404. doi: 10.1037/a0031474

Irwin, A., Li, J., Craig, W., \& Hollenstein, T. (2016). The role of shame in relation between peer victimization and mental health outcomes. Journal of Interpersonal Violence, 1-26. doi:10.1177/0886260516672937

Ivarsson, D., Bloom, M., Hesser, H., Carlbring, P., Enderby, P., Nordberg, R., \& Andersson, G. (2014). Guided internet-delivered cognitive behavior therapy for post-traumatic stress disorder: A randomized controlled trial. Internet Interventions, 1, 33-40. doi: 10.1016/j.invent.2014.03.002

Jackobsen, J. C., Gluud, C., Wetterslev, J., Winkel, P. (2017). When and how should multiple imputation be used for handling missing data in randomized clinical trials - a practical guide with flowcharts. BMC Medical Research Methodology, 17. doi: 10.1186/s12874-017-0442-1

Jenness, J. L., Jager-Hyman, S., Heleniak, C., Beck, A. T., Sheridan, M. A., \& McLaughlin, K. A. Catastrophizing, rumination, and reappraisal prospectively predict adolescent PTSD symptom onset following a terrorist attack. Depression and Anxiety, 33, 1039-1047. doi:10.1002/da.22548

Johansson, R., \& Andersson, G. (2012). Internet-based psychological treatments for depression. Expert Review of Neurotherapeutics, 12, 861-870. doi:10.1586/ern.12.63

Jorm, A.F., Morgan, A.J., \& Hetrick, S.E. (2008). Relaxation for depression. Cochrane Database of Systematic Reviews, 4. doi:10.1002/14651858.CD007142.pub2 
Katapally, T. R., Thorisdottir, A.S., Laxer, R., Qian, W., Leatherdale, S. T. (2018). The association of school connectedness and bullying involvement with multiple screen-time behaviours among youth in two Canadian provinces: a COMPASS study. Health Promotion and Chronic Disease Prevention in Canada: Research, Policy, and Practice, 10, 368-379. doi:10.24095/hpcdp.38.10.03

Klomek, A. B., Sourander, A., Niemelä, S., Kumpulainen, K., Piha, J., Tamminen, T. ... Gould, M. S. (2009). Childhood bullying behaviors as a risk for suicide attempts and completed suicides: A population-based birth cohort study. Journal of the American Academy of Child \& Adolescent Psychiatry, 48, 254-261. doi:10.1097/CHI.0b013e318196b91f

Kraemer, K.M., Luberto, C. M., O’Bryan, E.M., Mysinger, E., \& Cotton, S. (2016). Mind-body skills training to improve distress tolerance in medical students: a pilot study. Teaching and Learning in Medicine, 28, 219-228. doi:10.1080/10401334.2016.1146605

Kreft, I., \& DeLeeuw, J. (1998). Introducing Multilevel Modeling. Thousand Oaks, CA: Sage.

Kumpulainen, K. \& Räsänen, E. (2000). Children involved in bullying at elementary school age: Their psychiatric symptoms and deviance in adolescence. Child Abuse and Neglect, 24, 1567-1577. doi:10.1016/s0145-2134(00)00210-6

Ladd, B. K., \& Ladd, G. W. (2001). Variations in peer victimization: Relations to children's maladjustment. In J. Juvonen \& S. Graham (Eds.), Peer Harassment in School: The Plight of the Vulnerable and Victimized (3-21). New York: The Guilford Press. 
Lazarus, R. S., \& Folkman, S. (1984). Stress, appraisal, and coping. New York: Springer.

Leka, S., Vartia, M.., Hassard, J., Pahkin, K., Sutela, S., Cox, T., \& Lindstrom, K. (2008). Best practice in interventions for the prevention and management of workrelated stress and workplace violence and bullying. In Leka S. \& Cox, T. (Eds.), The European Framework for Psychological Risk Management (136 -173). Nottingham, UK: PRIMA-EF.

Lereya, S. T., Winsper, C., Heron, J., Lewis, G., Gunnell, D., Fisher, H. L., \& Wolke, D. (2013). Being bullied during childhood and the prospective pathways to selfharm in late adolescence. Journal of the American Academy of Child \& Adolescent Psychiatry, 52, 608-618. doi:10.1016/j.jaac.2013.03.012

Levinson, C. A., Langer, J. K., \& Rodebaugh, R. L. (2013). Reactivity to exclusion prospectively predicts social anxiety symptoms in young adults. Behavior Therapy, 44, 470-478. doi:10.1016/j.beth.2013.04.0077967(99)00161-8

Linden, M. (2003). The posttraumatic embitterment disorder. Psychotherapy and Psychosomatics, 72, 195-202. doi:10.1159/000070783

Linden, M., Baumann, K., Rotter, M., \& Schippan, B. (2007). The Psychopathology of posttraumatic embitterment disorder. Psychopathology, 40, 159- 165. doi:10.1159/000100005

Linden, M., Baumann, K., Rotter, M., \& Schippan, B. (2009). Posttraumatic embitterment disorder in comparison to other mental disorders. Psychotherapy and Psychosomatics, 77, 50 - 56. doi:10.1159/000110060

Linden, M., Baumann, K., Lieberei, B., Lorenz, C., \& Rotter, M. (2011). Treatment of posttraumatic embitterment disorder with cognitive behaviour therapy based on 
wisdom psychology and hedonia strategies. Psychotherapy and Psychosomatics, 80, 199-205. doi:10.1159/000321580

Litman, J. A. (2006). The COPE inventory: Dimensionality and relationships with approach - and avoidance- motives and positive and negative traits. Personality and Individual Differences, 41, 273-284. doi:10.1016/j.paid.2005.11.032

Lovibond, S. H., \& Lovibond, P. F. (1995). Manual for the Depression Anxiety \& Stress Scales. (2nd ed.). Sydney, Australia: Psychology Foundation.

Maas, C. J., \& Hox, J.J. (2005). Sufficient sample sizes for multilevel modeling. Methodology, 1, 86-92. doi:10.1027/1614-2241.1.3.86

Marks, I., Lovell, K., Noshirvani, H., Livanou, M., \& Thrasher, S. (1998). Treatment of posttraumatic stress disorder by exposure and/or cognitive restructuring. Archieves of Generic Psychiatry, 55, 317-325.

Marshall-Berenz, E. C., Vujanovic, A. A., Bonn-Miller, M. O., Bernstein, A., \& Zvolensky, M. J. (2010). Multimethod study of distress tolerance and PTSD symptom severity in a trauma-exposed community sample. Journal of Traumatic Stress, 23, 623-630. doi:10.1002/jts.20568

McDougall, P., \& Vaillancourt, T. (2015). Long-term adult outcomes of peer victimization in childhood and adolescence: Pathways to adjustment and maladjustment. American Psychologist, 70, 300-310. doi:10.1037/a0039174

McEvoy, P. M., Mahoney, A . E. (2011). Achieving certainty about the structure of intolerance of uncertainty in a treatment-seeking sample with anxiety and depression. Journal of Anxiety Disorders, 112-122.

doi:10.1016/j.janxdis.2010.08.010 
McHugh, R. K., Kertz, S. J., Weiss, R. B., Baskin-Sommers, A. R., Hearon, B.A., Björgvinsson, T. (2014). Changes in distress intolerance and treatment outcome in a partial hospital setting. Behavior Therapy, 45, 232-240. doi:10.1016/j.beth.2013.11.002

McMillan, K. A., Thorisdottir, A. S., \& Asmundson, G. J. G. (in press). Psychiatric correlates of past year adult bullying behaviors: findings from the national epidemiology survey of alcohol and related conditions. Psychiatry Research. doi:10.1016/j.psychres.2016.07.065

McNally, R., J. (2003). Remembering trauma. Cambridge, MA: The Belknap Press of Harvard University Press.

Mebane, S. (2010). Adult survivors of adolescent bullying: An exploration of long term effects (Unpublished doctoral dissertation). University of Illinois, Urbana, Illinois.

Medina, J., Hopkins, L., Powers, M., Baird, S.O., Smits, J. (2015). The effects of a hatha yoga intervention on facets of distress tolerance. Cognitive Behaviour Therapy, 44, 288-300. doi:10.1080/16506073.2015.1028433.

Menatti, A. R., Weeks, J. W., Carleton, R. N., Morrison, A. S., Heimberg, R. G., Hope, D. A., ...Liebowitz. (2015). The social interaction phobia scale: Continued support for the psychometric validity of the SIPS using clinical and non-clinical samples. Journal of Anxiety Disorders, 32, 46-55. doi:10.1016/j.janxdis.2015.03.003

Merrell, K. W., Gueldner, B. A., Ross, S. W., \& Isava, D. M. (2008). How effective are school bullying intervention programs? A meta-analysis of intervention research. School Psychology Quarterly, 23, 26-42. doi:10.1037/1045-3830.23.1.26 
Michailidis, E., \& Cropley, M. (2019). Testing the benefits of expressive writing for workplace embitterment: a randomized control trial. European Journal of Work and Organizational Psychology. doi:10.1080/1359432X.2019.1580694

Mol, S., S., L., Arntz, A., Metsemakers, J. F. M., Diant, G., Vilters-Van Montfort, P. A. P., Knottnerus, J. A. (2005). Symptoms of post-traumatic stress disorder after non-traumatic events: evidence from an open population study. The British Journal of Psychiatry, 186, 494-499. doi:10.1192/bjp.186.6.494

Monteleone, P., Nolfe, G., Serritella, C., Milano, C., Di Cerbo, A., Blasi, F...\& Maj, M. (2009). Hypoactivity of the hypothalamo-pituitary-adrenal axis in victims of mobbing: role of subjects' temperament and chronicity of the work-related psychological distress. Psychotherapy and Psychosomatics, 78, 381-383. doi:10.1159/000235980

Mulder, R., Fergusson, D., \& Horwood, J. (2013). Post-traumatic stress disorder symptoms form a traumatic and non-traumatic stress response dimension. Australian \& New Zealand Journal of Psychiatry, 47, 569-577. doi:10.1177/0004867413484367

Nansel, T. R., Craig, W., Overpeck, M.D., Saluja, G., \& Ruan, J. (2004). Cross-national consistency in the relationship between bullying behaviors and psychosocial adjustment. Archives of Pediatrics \& Adolescent Medicine, 158, 730-736. doi:10.1001/archpedi.158.8.730

Nielsen, M. B., Matthiesen, S. B., \& Einarsen, S. (2010). The impact of methodological moderators on prevalence rates of workplace bullying. A meta-analysis. Journal 
of Occupational and Organizational Psychology, 83, 955 - 979. doi:10.1348/096317909X481256

Nielsen, M. B.; Tangen, T., Idsoe, T., Matthiesen, S. B., \& Magerøy, N. (2015). Posttraumatic stress disorder as a consequence of bullying at work and at school. A literature review and meta-analysis. Aggression and Violent Behavior, 21, 17-24. doi:10.1016/j.avb.2015.01.001

Nilsson, J. E., Lundh, L. G., Viborg, G. (2012). Imagery rescripting of early memories in social anxiety disorder: An experimental study. Behaviour Research and Therapy, 50, 387-392. doi:10.1016/j.brat.2012.03.004

Nishith, P., Nixon, R. D. V., Resick, P. A. (2005). Resolution of trauma-related guilt following treatment of PTSD in female rape victims: A result of cognitive processing therapy targeting comorbid depression? Journal of Affective Disorders, 86, 259-265. doi: 10.1016/j.jad.2005.02.013

Nishith, P., Resick, P.A., \& Griffin, M. G. (2002). Pattern of change in prolonged exposure and cognitive-processing therapy for female rape victims with posttraumatic stress disorder. Journal of Consulting and Clinical Psychology, 70, 880-886. doi:10.1037/0022-006X.70.4.880

Notalaers, G., Van der Hejden, B., Guenter, H., Nielsen, M. B., \& Einarsen, S. V. (2018). Do interpersonal conflict, aggression and bullying at the workplace overlap? A latent class modeling approach. Frontiers in Psychology, 9. doi:10.3389/fpsyg.2018.01743

Olthuis, J. V., Wozney, L., Asmundson, G. J. G., Cramm, H., Lingley-Pottie, P., \& McGrath, P. (2016). Distance-delivered interventions for PTSD: a systematic 
review and meta-analysis. Journal of Anxiety Disorders, 44, 9-26.

doi:10.1016/j.janxdis.2016.09.010

Orth, U., \& Wieland, E. (2006). Anger, hostility, and posttraumatic stress disorder in trauma-exposed adults: a meta-analysis. Journal of Consulting and Clinical Psychology, 74, 698-706. doi:10.1037/0022-006X.74.4.698

Olweus, D. (1994). Bullying at school: Basic facts and effects of a school based intervention program. Journal of Child Psychology and Psychiatry, 35, 11711190. doi:10.1111/j.1469-7610.1994.tb01229.x

Olweus, D. (2001). Peer harassment: a critical analysis and some important issues. In J. Juvonen \& S. Graham (Eds.), Peer Harassment in School: The Plight of the Vulnerable and Victimized (3-21). New York: The Guilford Press.

Ozer, E., Best, S., Lipsey, T., \& Weiss, D. (2008). Predictors of posttraumatic stress disorder and symptoms in adults: a meta-analysis. Psychological Bulletin, 129, 52-73. doi:10.1037//0033-2909.129.1.52

Pawlow L. A., \& Jones, G., E. (2002). The impact of abbreviated progressive muscle relaxation on salivary cortisol. Biological Psychology, 60, 1-16. doi:10.1016/s0301-0511(02)00010-8

Pihlaja, S., Stenberg, J. H., Joutsenniemi, K., Mehik, H., Ritola, V., \& Joffe, G. (2018). Therapeutic alliance in guided internet therapy programs for depression and anxiety disorders - a systematic review. Internet Interventions, 11, 1-10. doi:10.1016/j.invent.2017.11.005

Price, J. L., MacDonald, H. Z., Adair, K. C., Koerner, N., \& Monson, C. M. (2014). Changing beliefs about trauma: A qualitative study of cognitive processing 
therapy. Behavioural and Cognitive Psychotherapy, 1-12.

doi:10.1017/S1352465814000526

Rachman, S., Grüter- Andrew, J. \& Shafran, R. (2000). Post-event processing in social anxiety. Behaviour Research and Therapy, 38, 611-617. doi:10.1016/S00057967(99)00089-3

Reiss, S., \& McNally, R. J. (1985). Expectancy model of fear. In S. Reiss \& R. R. Bootzin (Eds.), Theoretical Issues in Behavior Therapy (pp. 107-121). San Diego, CA: Academic Press.

Resick, P. A., \& Schnicke, M. A. (1992). Cognitive processing therapy for sexual assault victims. Journal of Consulting and Clinical Psychology, 60, 748-756. doi:10.1037//0022-006x.60.5.748

Resick, P. A., Nishith, P., Weaver, T. L., Astin, M. C., Feuer, C. A. (2002). A comparison of cognitive-processing therapy with prolonged exposure and a waiting condition for the treatment of chronic posttraumatic stress disorder in female rape victims. Journal of Consulting and Clinical Psychology, 70, 867879. doi:10.1037//0022-006x.70.4.867

Resick, P. A., Galovski, T. E., O’Brien Uhlmansiek, M., Scher, C. D., Clum, G. A., Young-Xu, Y. (2008). A randomized clinical trial to dismantle components of cognitive processing therapy for posttraumatic stress disorder in female victims of interpersonal violence. Journal of Consulting and Clinical Psychology, 76, 243-258. doi:10.1037/0022-006X.76.2.243 
Resick, P. A., Monson, C. M., \& Chard, K. M. (2014). Cognitive Processing therapy: Veteran/Military version: Therapist's manual. Washington, DC: Department of Veterans Affair

Rizvi, S. L., Vogt, D. S., \& Resick, P. A. (2012). Cognitive and affective predictors of treatment outcome in cognitive processing therapy and prolonged exposure for posttraumatic stress disorder. Behaviour Research and Therapy, 47, 737-743. doi: 10.1016/j.brat.2009.06.003

Roland, A. G., Currier, J. M., Rojas-Flores, L., \& Herrera, S. (2014). Event centrality and posttraumatic outcomes in the context of pervasive violence: A study of teachers in El Salvador. Anxiety, Stress \& Coping: An International Journal, 27, 335-346. doi:10.1080/10615806.2013.835402

Salin, D., Cowan, R., Adewumi, O., Apospori, E., Bochantin, J., D’Cruz, P., ...Zedlacher, E. (2019). Workplace bullying across the globe: a cross-cultural comparison. Personnel Review, 48, 204-219. doi:10.1108/PR-03-2017-0092

Sari, S. V., \& Camadan, F. (2016). The new face of violence tendency: Cyber bullying perpetrators and their victims. Computers in Human Behavior, 59, 317-326. doi:10.1016/j.chb.2016.02.027

Salmivalli, C., \& Nieminen, E. (2002). Proactive and reactive aggression among school bullies, victims, and bully-victims. Aggressive Behavior, 28, 30-44. doi:10.1002/ab.90004.abs

Schneider, L. H., Hadjistavropoulos, H. D., Faller, Y. N. (2016). Internet-delivered cognitive behaviour therapy for depressive symptoms: An exploratory examination of therapist behaviours and their relationship to outcome and 
therapeutic alliance. Behavioural and Cognitive Psychotherapy, 44, 625-639. doi: $10.1017 / S 1352465816000254$

Schuettler, D., \& Boals, A. (2011). The path to posttraumatic growth versus posttraumatic stress disorder: Contributions of event centrality and coping. Journal of Loss and Trauma, 16, 180-194. doi:10.1080/15325024.2010.519273

Schulz, K. F., Altman, D. G., \& Moher, D. (2010). CONSORT 2010 statement: Updated guidelines for reporting parallel group randomised trials. BMC Medicine, 8. doi:10.1016/j.ijsu.2011.09.004

Schulz, A., Vincent, A., \& Berger, T. (2017). Daydreamer and night owl: comparing positive and negative outcome cases in an online, clinician-guided, self-help intervention for social anxiety disorder. Pragmatic Case Studies in Psychotherapy, 13, 217-252. doi:10.14713/pcsp.v13i3.2012

Schäfer, M., Korn, S., Smith, P. K., Hunter, S. C., Mora-Merchán, J. A., Singer, M. M., et al. (2004). Lonely in the crowd: Recollections of bullying. British Journal of Developmental Psychology, 22, 379-394. doi:10.1348/0261510041552756

Shipherd, J. C., Street, A. E., Resick, P. A. (2006). Cognitive therapy for posttraumatic stress disorder. In: Follette, V. M. \& Ruzek, J. I., Eds. Cognitive-behavioral therapies for trauma. New York (pp. 96-116), NY: Guilford Press

Shireen, F., Janapana, H., Rehmatullah, S., Temuri, H. \& Azim, F. (2014). Trauma experience of youngsters and teens: A key issue in suicidal behavior among victims of bullying? Pakistan Journal of Medical Science, 30, 206-210. doi:10.12669/pjms.301.4072 
Siegel, R. S., La Greca, A. M., Harrison, H. M. (2009). Peer victimization and social anxiety in adolescents: Prospective and reciprocal relationships. Journal of Youth and Adolescene, 38, 1096-1109. doi:10.1007/s10964-009-9392-1

Sijbrandij, M., Kunovksi, I., \& Cuijpers, P. (2016). Effectiveness of internet-delivered cognitive behavioural therapy for posttraumatic stress disorder: A systematic review and meta-analysis. Depression and Anxiety, 33, 783-791. doi: $10.1002 /$ da. 22533

Simons, J. S., \& Gaher, R. M. (2005). The distress tolerance scale: Development and validation of a self-report measure. Motivation and Emotion, 29, 83-102. doi:10.1007/s11031-005-7955-3

Smith, P. K., Shu, S., \& Madsen, K. (2001). Characteristics of victims of school bullying in developing changes in coping strategies and skills. In Juvonen J., Graham S. editors. Peer Harassment in school: The plight of the vulnerable and victimized. New York: Guilford Press, p. 332-351.

Smith, P. K., Talamelli, L., Cowie, H., Naylor, P., \& Chauhan, P. (2004). Profiles of nonvictims, escaped victims, continuing victims and new victims of school bullying. British Journal of Educational Psychology, 74, 565-581. doi:10.1348/0007099042376427

Smokovski, P. R., \& Kopasz, K. H. (2005). Bullying in school: An overview of types, effects, family characteristics, and intervention strategies. Children \& Schools, 27, 101-110. doi:10.1093/cs/27.2.101

Sourander, A., Jensen, P., Rönning, J. A., Niemelä, S., Helenius, H., Sillanmäki, L. ... Almqvist, F. (2007). What is the early adulthood outcome of boys who bully or 
are bullied in childhood? The Finnish "from a boy to a man" study. Pediatrics, 120, 397-404. doi:10.1542/peds.2006-2704

Sourander, A., Ronning, J., Brunstein-Klomek, A., Gyllenberg, D., Kumpulainen, K., Niemela, S., ...Almqvist, F. (2009). Childhood bullying behavior and later psychiatric hospital and psychopharmacologic treatment: Findings from the Finnish 1981 birth cohort study. Archieves of General Psychiatry, 66, 10051012. doi:10.1001/archgenpsychiatry.2009.122

Startup, M., Makgekgenene, L., Webster, R. (2007). The role of self-blame for trauma as assessed by the posttraumatic cognitions inventory (PTCI): a self-protective cognition? Behaviour Research and Therapy, 45, 395 - 403. doi:10.1016/j.brat.2006.02.003

Strøm, I. F., Aakvaag, H. F., Birkeland, M.S., Felix, E., \& Thoresen, S. (2018). The mediating role of shame in the relationship between childhood bullying victimization and adult psychosocial adjustment. European Journal of Psychotraumatology, 9. doi.org/10.1080/20008198.2017.1418570

Tabachnick, B. G. \& Fidell, L. S. (2013). Using Multivariate Statistics, 6th ed. Boston: Pearson.

Takizawa, R., Maughan, B., \& Arseneault L. (2014). Adult health outcomes of childhood bullying victimization: Evidence from a five-decade longitudinal British Birth Cohort. American Journal of Psychiatry, 171, 777-784. doi:10.1176/appi.ajp.2014.13101401

Taylor, S., (2017). Clinician's guide to PTSD: a cognitive-behavioural approach (2 ${ }^{\text {nd }}$ ed.). New York, NY: The Guilford Press 
Taylor, K. A., Sullivan, T. N., \& Kliewer, W. (2013). A longitudinal path analysis of peer victimization, threat appraisals to self, and aggression, anxiety, and depression among urban African American adolescents. Journal of Youth and Adolescence, 42, 178-789. doi:10.1007/s10964-012-9821-4

Taylor, S., Fedoroff, I. C., Koch, W. J., Thordarson, D. S., Fecteau, G., \& Nicki, R. M. (2001). Posttraumatic stress disorder arising after road traffic collisions: Patterns of response to cognitive-behavior therapy. Journal of Consulting and Clinical Psychology, 69, 541-551. doi:10.1037//0022-006x.69.3.541

Taylor, S., Thordarson, D. S., Maxfield, L., Fedoroff, I. C., Lovell, K., \& Ogrodniczuk, J. (2003). Comparative efficacy, speed, and adverse effects of three PTSD treatments: Exposure therapy, EMDR, and relaxation training. Journal of Consulting and Clinical Psychology, 71, 330-338. doi: 10.1037/0022006X.71.2.330

Taylor, S., Zvolensky, M. J., Cox, B. J., Deacon, B., Heimberg, R. G., Ledley, D. R., ... Cardenas, S. J. (2007). Robust dimensions of anxiety sensitivity: Development and initial validation of the Anxiety Sensitivity Index-3. Psychological Assessment, 19, 176-188. doi:10.1037/1040-3590.19.2.176

Ttofi, M. M., \& Farrington, D. P. (2011). Effectiveness of school-based programs to reduce bullying: A systematic and meta-analytic review. Journal of Experimental Criminology, 7, 27-56. doi:10.1007/s11292-010-9109-1

Ttofi, M. M., Farrington, D. P., \& Lösel, F. (2014). Protective factors interrupting the continuity from school bullying to later internalizing and externalizing 
problems: A systematic review of prospective longitudinal studies. Journal of School Violence, 13, 5-38. doi:10.1080/15388220.2013.857345

Unnever, J. D., \& Cornell, D. G. (2004). Middle school victims of bullying: Who reports being bullied. Aggressive behavior, 30, 373-388. doi:10.1002/ab.20030

Vallancourt, T., Hymel, S., \& McDougall, P. (2013). The biological underpinnings of peer victimization: Understanding why and how the effects of bullying can last a lifetime. Theory into Practice, 52, 241-248.

doi:10.1080/00405841.2013.829726

van der Kolk, B. A. (2007). The history of trauma in psychiatry. In: M. J. Friedman, T. M. Keane \& P.A. Resick (Eds.). Handbook of PTSD: Science and Practice (pp. 1937). New York, NY: The Guilford Press.

van Minnen, A., Zoellner, L. A., Harned, M. S., \& Mills, K. (2015). Changes in comorbid conditions after prolonged exposure for PTSD: a literature review. Current Psychiatry Reports, 17. doi:10.1007/s11920-015-0549-1

Vartia, M., \& Leka, S. (2011). Interventions for the prevention and management of bullying at work. In Bullying and Harassment in the Workplace: Developments in Theory, Research and Practice ( $2^{\text {nd }}$ ed.). Taylor \& Francis: Boca Raton, FL, USA.

Varvogli, L., \& Darviri, C. (2011). Stress management techniques: evidence-based procedures that reduce stress and promote health. Health Science Journal, 5(2), 74-89. 
Vega, G. \& Comer, D.R. (2005). Sticks and stones may break your bones, but words can break your spirit: Bullying in the workplace. Journal of Business Ethics, 58, 101-109. doi:10.1007/s10551-005-1422-7

Veenstra, R., Lindenberg, S., Oldehinkel, A. J., De Winter, A. F., \& Verhulst, F. C. (2003). Bullying and victimization in elementary schools: A comparison of bullies, victims, bully/victims, and uninvolved preadolescents. Developmental Psychology, 41, 672-682. doi:10.1037/0012-1649.41.4.672

Verkuil, B., Atasayi, S., Molendijk, M. L. (2015). Workplace bullying and mental health: a meta-analysis on cross-sectional and longitudinal data. PLOS ONE, 8, e0135225. doi: 10.1371/journal.pone.0135225pone.0135225

Walter, K., Bolte, T., Owens, G., \& Chard, K. (2012). The impact of personality disorders on treatment outcome for veterans in a posttraumatic stress disorder residential treatment program. Cognitive Therapy and Research, 36, 576-584. doi: 10.1007/s10608-011-9393-8

Weathers, F. W., Litz, B.T., Keane, T.M., Palmieri, P.A., Marx, B.P., \& Schnurr, P.P. (2013). The PTSD Checklist for DSM-5 (PCL-5). Scale available from the National Center for PTSD at www.ptsd.va.gov.

Wenzel, A. (2012). Modification of core beliefs in cognitive therapy. In Irismar Reis De Oliveira (Ed.), Standard and Innovative Strategies in Cognitive Behaviour Therapy ( ISBN: 978-953-51-0312-), InTech: London. doi:10.5772/1161

Wild, J., Hackmann, A., \& Clark, D. M. (2008). Rescripting early memories linked to negative images in social phobia: A pilot study. Behavior Therapy, 39, 47-56. doi:10.1016/j.beth.2007.04.003 
Wild, J. \& Clark, D. M. (2011). Imagery rescripting of early traumatic memories in social phobia. Cognitive and Behavioral Practice, 18, 433-443. doi:10.1016/j.cbpra.2011.03.002

Williams, S. L., Williams, D. R., Stein, D. J., Seedat, S., Jackson, P. B. \& Moomal, H. (2007). Multiple traumatic events and psychological distress: The South Africa stress and health study. Journal of Traumatic Stress, 20, 845-855. doi:10.1002/jts.20252

Wilson, J. K., \& Rapee, R. M. (2005). The interpretation of negative social events in social phobia: Changes during treatment and relationship to outcome. Behaviour Research and Therapy, 43, 373-389. doi:10.1016/j.brat.2004.02.006

Woltman, H., Feldstain, A., MacKay, J. C., \& Rocchi, M. (2012). An introduction to hierarchical linear modeling. Tutorials in Quantitative Methods for Psychology, 8, 52-69. doi:10.20982/tqmp.08.1.p052

Woods, A. P., Stults, C. R., Terry, R. L., \& Rego, S. A. (2017). Strengths and limitations of internet-based cognitive behavioural treatments for anxiety disorders.

Pragmatic Case Studies in Psychotherapy, 13, 271-283. doi:10.14713/pcsp.v13i3.2015

Young, C., \& Campbell, K. (2018). Internet-delivered cognitive behavioural therapy for post-traumatic stress disorder: A review of clinical effectiveness. CADTH rapid response report: Peer-reviewed summary with critical appraisal: Ottawa.

Retrieved from:

https://www.ncbi.nlm.nih.gov/books/NBK538921/pdf/Bookshelf_NBK538921. pdf 
Zappert, L. N., Westrup, D. (2008). Cognitive processing therapy for posttraumatic stress disorder in a residential treatment setting. Psychotherapy: Theory, Research, Practice, Training, 45, 361-376. doi:10.1037/0033-3204.45.3.361

Zimet, G. D., Dahlem, N. W., Zimet, S. G., \& Farley, G. K. (1988). The Multidimensional scale of perceived social support. Journal of Personality Assessment, 52(1), 30-41. doi:10.1207/s15327752jpa5201_2

Zvolenski, M. J., Vujanovic, A. A., Bernstein, A., \& Leyro, T. (2010). Distress tolerance: Theory, measurement and relations to psychopathology. Current Directions in Psychological Science, 19, 406-410. doi:10.1177/0963721410388642 


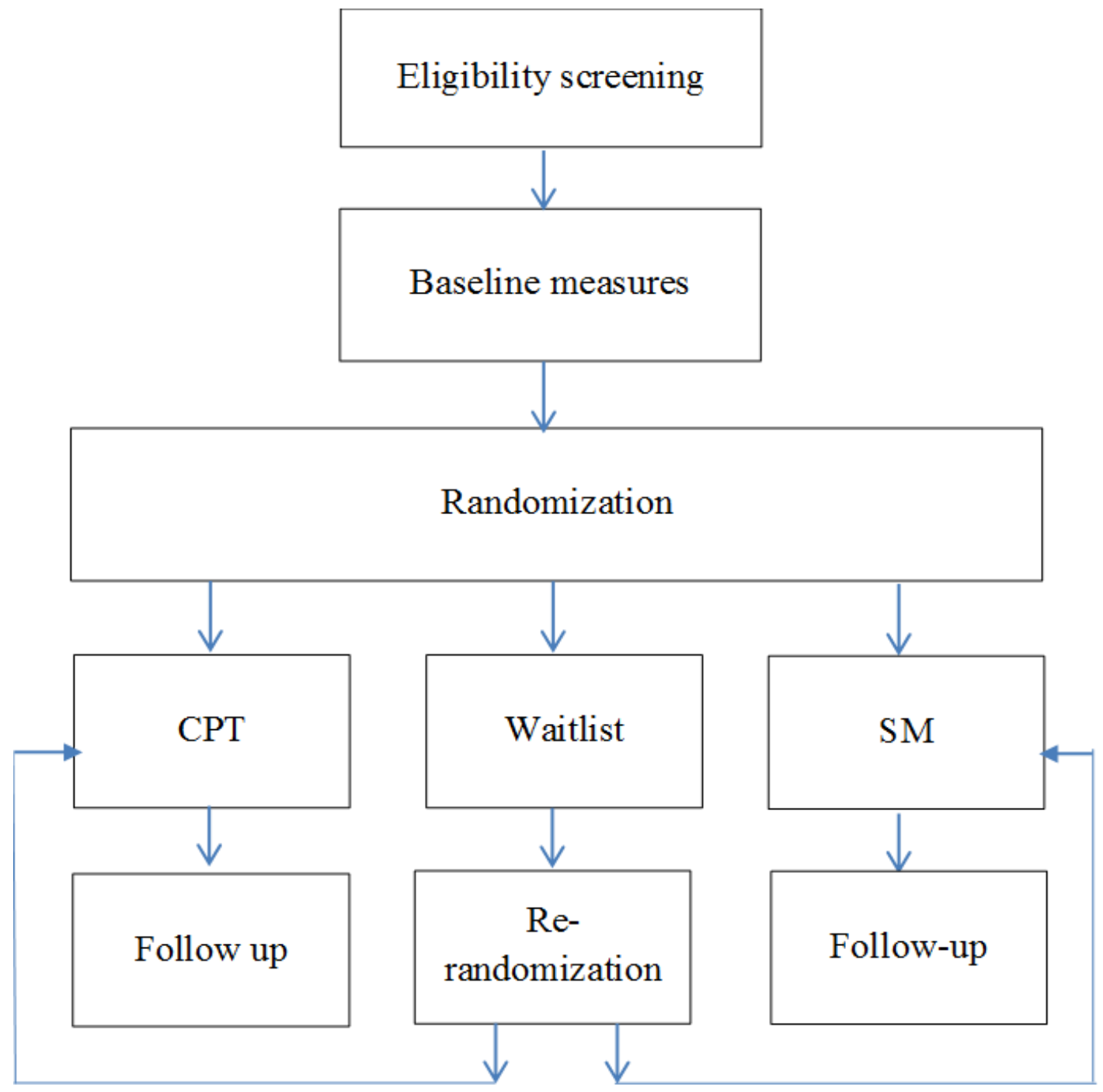

Figure 1: Study procedures 


\section{APPENDICES}

\section{Appendix A: Certificate of Research Approval}

\section{University of Regina

\author{
Research Ethics Board \\ Certificate of Approval
}

PRINCIPAL INVESTIGATOR

DEPARTMENT

REB\#

Audur Sjofn Thorisdottir

Psychology

2017-063

SUPERVISOR: Dr. Gordon J. G. Asmundson

TITLE

Internet Delivered Cognitive Processing Therapy for Individuals with a History of Bullying Victimization: A Randomized Controlled Trial

APPROVED ON: April 24, 2017

RENEWAL DATE: April 24, 2018

APPROVAL OF:

Application For Behavioural Research Ethics Review

Depression Anxiety Stress Scales

Recruitment Poster

Consent Form

Demographic Questions

Retrospective Bullying Questionnaire

Life Events Checklist- 5

P4-Screener

Posttraumatic Stress Disorder Checklist

Posttraumatic Cognitions Inventory

Centrality of Event Scale

Social Interaction Phobia Scale

Dimensions of Anger Reactions-5 Scale

Distress Tolerance Scale

Anxiety Sensitivity Index-3

Intolerance of Uncertainty Scale, Short Form

Cognitive Emotion Regulation Questionnaire, Short Form

Brief Resilience Scale

Multidimensional Scale of Perceived Social Support

Cognitive Processing Therapy for Social Trauma

Stress Management Intervention

Full Board Meeting

Delegated Review

The University of Regina Research Ethics Board has reviewed the above-named research project. The proposal was found to be acceptable on ethical grounds. The principal investigator has the responsibility for any other administrative or regulatory approvals that may pertain to this research project, and for ensuring that the authorized research is carried out according to the conditions outlined in the original protocol submitted for ethics review. This Certificate of Approval is valid for the above time period provided there is no change in experimental protocol, consent process or documents.

Any significant changes to your proposed method, or your consent and recruitment procedures should be reported to the Chair for Research Ethics Board consideration in advance of its implementation.

\section{ONGOING REVIEW REQUIREMENTS}

In order to receive annual renewal, a status report must be submitted to the REB Chair for Board consideration within one month of the current expiry date each year the study remains open, and upon study completion. Please refer to the following website for further instructions: http://www.uregina.ca/research/for-faculty-staff/ethics-

compliance/human/forms1/ethics-forms.html.

Dr. Katherine Robinson

Chair, Research Ethics Board 


\section{Appendix B: Renewal of Research Approval Certificate}

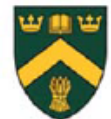

PRINCIPAL INVESTIGATOR

EPARTMENT

REB\#

Audur Sjofn Thorisdottir

Psychology

2017-063

SUPERVISOR: Dr. Gordon J. G. Asmundson

TITLE

Internet Delivered Cognitive Processing Therapy for Individuals with a History of Bullying Victimization: A Randomized Controlled Trial

ORIGINAL DATE OF

APPROVAL:

April 24, 2017
NEW EXPIRY DATE WITH

THIS RENEWAL:

April 24, 2019
TODAY'S

DATE:

May 2, 2018

Full Board Meeting

Delegated Review

凶

\section{RENEWAL CERTIFICATION}

The University of Regina Research Ethics Board has renewed the above-named research project for an additional 12 months beginning April 24, 2018.

Any significant changes to your proposed method, or your consent and recruitment procedures should be reported to the Chair of the Research Ethics Board for consideration in advance of implementation.

\section{ONGOING REVIEW REQUIREMENTS}

In order to receive annual renewal, a status report must be submitted to the REB Chair for Board consideration within one month of the current expiry date each year the study remains open, and upon study completion. Please refer to the following website for further instructions:

https://www.uregina.ca/research/for-faculty-staff/ethics-compliance/human/ethics-forms.html

Ara Steininger

Research Ethics Board 
Appendix C: Certificate of Approved Amendment to Research Approval

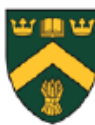

University of Regina

Research Ethics Board

Certificate of Amendment Approval

PRINCIPAL INVESTIGATOR:

DEPARTMENT:

REB\#:

Auder Thorisdottir

Department of Psychology

2017-063

TITLE: Internet Delivered Cognitive Processing Therapy for Individuals with a History of Bullying Victimization: A Randomized Controlled Trial

AMENDMENT APPROVAL OF:

- Reach out to participants for content feedback

NEXT RENEWAL

AMENDMENT

DATE

April 24, 2019 APPROVAL DATE

- Addition of Cognitive Processing Therapy Content Survey and Stress Management Program Survey

Full Board Meeting

Delegated Review

$凶$

\section{AMENDMENT CERTIFICATION}

The University of Regina Research Ethics Board has reviewed the changes to the above-named research project as outlined in your memo dated January 4, 2018, and they are approved.

ONGOING REVIEW REQUIREMENTS

In order to receive annual renewal, a status report must be submitted to the REB Chair for Board consideration within one month of the current expiry date each year the study remains open, and upon study completion. Please refer to the following website for the renewal and closure forms:

https://www.uregina.ca/research/for-faculty-staff/ethics-compliance/human/ethicsforms.html

Ara Steininger

Research Ethics Board

Fax: (306) 585-4893

research.ethics@uregina.ca 
Appendix D: The Retrospective Bullying Questionnaire

The following questions are about bullying. Bullying is intentional hurtful behaviour. It can be physical or psychological. It is often repeated and characterized by an inequality of power so that it is difficult for the victim to defend him/her self.

Please think back to your school days. You may have seen some bullying at school, and you may have been involved in some way. Which choice(s) best descripe your own experience at school?

I was not involved at all, and I never saw it happen

I was not involved at all, but I saw it happen sometimes

I would sometimes join in bullying others

I would sometimes get bullied by others

At various times, I was both a bully and a victim

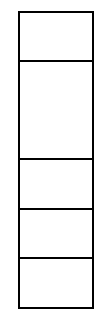

\section{PART I: PRIMARY EDUCATION.}

This part deals with your primary education experiences (4-11 years).

Please circle the answer that is right for you.

1. Did you have a happy time during your primary education?

detested disliked neutral liked a bit liked a lot

2. Did you have a happy time at home with your family during your primary education?

detested disliked neutral liked a bit liked a lot

The next questions are about physical forms of bullying - hitting and kicking, and having things stolen from you.

3. Were you physically bullied during your primary education?

Hit/punched

Stolen from

Other

\begin{tabular}{|l|l|}
\hline yes & no \\
\hline & \\
\hline & \\
\hline & \\
\hline
\end{tabular}

4. Did this happen?

never rarely sometimes frequently constantly

5. How serious did you consider these bullying-attacks to be?

I wasn't bullied not at all only a bit quite serious extremely serious 
The next questions are about verbal forms of bullying - being called nasty names, and being threatened.

6. Were you verbally bullied during your primary education?

Called names

Threatened

Other

\begin{tabular}{|l|l|}
\hline yes & no \\
\hline & \\
\hline & \\
\hline & \\
\hline
\end{tabular}

7. Did this happen?

never rarely sometimes frequently constantly

8. How serious did you consider these bullying-attacks to be?

I wasn't bullied not at all only a bit quite serious extremely serious

The next questions are about indirect forms of bullying - having lies or nasty rumours told about behind your back, or being deliberately excluded from social groups.

9. Were you indirectly bullied during your primary education?

Had lies told about you Excluded

Other

\begin{tabular}{|l|l|}
\hline yes & no \\
\hline & \\
\hline & \\
\hline & \\
\hline
\end{tabular}

10. Did this happen?

never rarely sometimes frequently constantly

11. How serious did you consider these bullying-attacks to be?

I wasn't bullied not at all only a bit quite serious extremely serious

The next questions are about cyber-bullying - having lies, nasty rumours or pictures spread about on the internet, nasty messages or emails sent to you.

12. Were you cyber-bullied during your primary education?

Rumours spread online

Pictures sent around

Nasty messages

Other

\begin{tabular}{|l|l|}
\hline yes & no \\
\hline & \\
\hline & \\
\hline & \\
\hline & \\
\hline
\end{tabular}


13. Did this happen?

never rarely sometimes frequently constantly

14. How serious did you consider these cyber bullying-attacks to be?

I wasn't bullied not at all only a bit quite serious extremely serious

The next questions are about bullying in general during your primary education.

15. How long did the bullying attacks usually last?

I wasn't bullied just a few days weeks months a year or more

16. How many students bullied you during your primary education?

I wasn't bullied

Mainly by one boy

By several boys

Mainly by one girl

By several girls

By both boys and girls

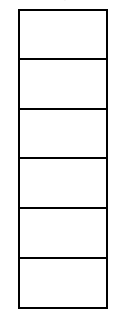

17. If you were bullied, why do you think this happened?

\section{PART II: SECONDARY EDUCATION}

This part deals with your secondary education experiences (12-18 years).

Please circle/check the answer that is right for you.

18. Did you have a happy time during your secondary education?

detested disliked neutral liked a bit liked a lot

19. Did you have a happy time at home with your family during your secondary education?

detested disliked neutral liked a bit liked a lot 
The next questions are about physical forms of bullying - hitting and kicking, and having things stolen from you.

20. Were you physically bullied during your secondary education?

Hit/punched

Stolen from

Other

\begin{tabular}{|l|l|}
\hline yes & no \\
\hline & \\
\hline & \\
\hline & \\
\hline
\end{tabular}

21. Did this happen?

never rarely sometimes frequently constantly

22. How serious did you consider these bullying-attacks to be?

I wasn't bullied not at all only a bit quite serious extremely serious

The next questions are about verbal forms of bullying - being called nasty names, and being threatened.

23. Were you verbally bullied during your secondary education?

\begin{tabular}{l|l|l|}
\cline { 2 - 3 } Called names & yes & no \\
\cline { 2 - 3 } & & \\
\hline Threatened & & \\
\cline { 2 - 3 } Other & & \\
\cline { 2 - 3 }
\end{tabular}

24. Did this happen?

never rarely sometimes frequently constantly

25. How serious did you consider these bullying-attacks to be?

I wasn't bullied not at all only a bit quite serious extremely serious

The next questions are about indirect forms of bullying - having lies or nasty rumours told about behind your back, or being deliberately excluded from social groups.

26. Were you indirectly bullied during your secondary education?

Had lies told about you

Excluded

Other

\begin{tabular}{|l|l|}
\hline yes & no \\
\hline & \\
\hline & \\
\hline & \\
\hline
\end{tabular}


27. Did this happen?

never rarely sometimes frequently constantly

28. How serious did you consider these bullying-attacks to be?

I wasn't bullied not at all only a bit quite serious extremely serious

The next questions are about cyber-bullying - having lies, nasty rumours or pictures spread about on the internet, nasty messages or emails sent to you.

29. Were you cyber-bullied during your secondary education?

Rumours spread online

Pictures sent around

Nasty messages

Other

\begin{tabular}{|l|l|}
\hline yes & no \\
\hline & \\
\hline & \\
\hline & \\
\hline & \\
\hline
\end{tabular}

30. Did this happen?

never rarely sometimes frequently constantly

31. How serious did you consider these cyber bullying-attacks to be?

I wasn't bullied not at all only a bit quite serious extremely serious

The next questions are about bullying in general during your secondary education.

32. How long did the bullying attacks usually last?

I wasn't bullied just a few days weeks months a year or more

33. How many students bullied you during your secondary education?

I wasn't bullied

Mainly by one boy

By several boys

Mainly by one girl

By several girls

By both boys and girls

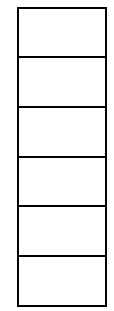

34. If you were bullied, why do you think this happened? 


\section{PART III: GENERAL EXPERIENCES AT SCHOOL}

35. Which were the main ways you used to to cope with the bullying? (Please check all that apply)

I wasn't bullied

I tried to make fun of it

I tried to avoid the situation

I tried to ignore it

I fought back

I got help from friends

Other

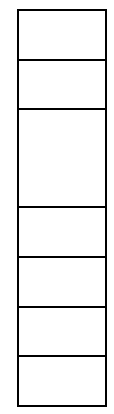

36. Which were the main ways you sought social support during the bullying experience? (Please check all that apply)

I felt supported by a friend/friends

I felt supported by my family

I felt supported by a teacher

I felt supported by my school

I did not feel supported Other

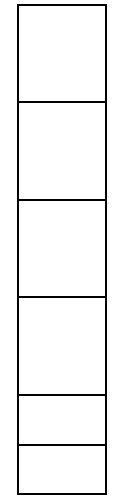

37. Did you ever take part in bullying anyone while you were at school?

Hit/punched

Stole from

Called names

Threatened

Told lies about

Excluded

\begin{tabular}{|l|l|}
\hline yes & no \\
\hline & \\
\hline & \\
\hline & \\
\hline & \\
\hline & \\
\hline & \\
\hline
\end{tabular}

38. Did this happen?

never rarely sometimes frequently constantly 
39. How often did you try to avoid school by pretending to be sick or by playing truant/skipped school because you were being bullied?

I wasn't bullied at school

Never

Only once or twice

Sometimes

Maybe once a week

Several times a week

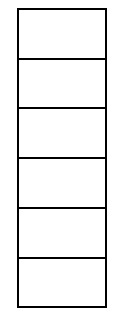

40. When you were being bullied, did you ever, even for a second, think about hurting yourself or taking your own life?

I wasn't bullied at school

No, never

Yes, once

Yes, more than once

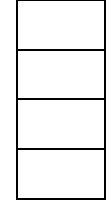

41. Have you been bullied since leaving school?

I haven't been bullied since leaving school

I have been bullied by my family

I have been bullied by others (please

specifcy

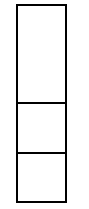

RECOLLECTIONS OF BEING BULLIED AT SCHOOL (Only answer these questions, if you were bullied)

42. Do you have vivid memories of the bullying event(s) which keep coming back causing you distress?

No, never not often sometimes often always

43. Do you have dreams or nightmares about the bullying event(s)?

No, never not often sometimes often always

44. Do you often feel like you are re-living the bullying event(s) again?

No, never not often sometimes often always

45. Do you ever have sudden vivid recollections or „flashbacks“ of the bullying event(s)?

No, never not often sometimes often always 
46. Do you ever feel distressed in situations which remind you of the bullying even(s)?

No, never not often sometimes often always

47. If you were bullied, do yo think it had any longterm consequences?

\section{PART IV: BULLYING OR HARASSMENT IN THE WORKPLACE/COLLEGE}

48. Have you ever experienced bullying in your workplace?

I wasn't bullied in my workplace

I was bullied in one of my previous jobs

I was bullied in more than one of my previous jobs

I have been bullied in my present job

I have been bullied in all of my jobs

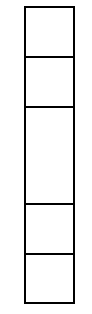

49. Please state whether you have been bullied at work over the last six months.

No, never

Yes, very rarely

Yes, now and then

Yes, several times per month

Yes, several times per week

Yes, almost daily

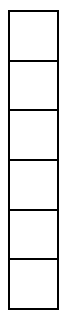

50. If yes, when did the bullying start?

Within the last 6 months 6-12 months ago 1-2 years ago More than 2 years ago

51. If you have been bullied, what did you do? Please check all that apply.

Tried to avoid the situation

Tried to ignore it

Confronted the bully

Went to union/staff association

Went to personnel

Discussed it with colleagues

Went to occupational health

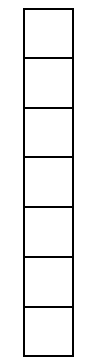


Went to the welfare department

Saw my doctor (GP)

I went for counseling

I got psychiatric help

I left the job

Did not really cope

Other

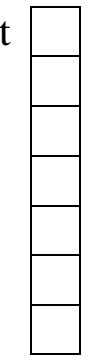


Appendix E: Life Events Checklist-5

\begin{tabular}{|c|c|c|c|c|c|}
\hline $\begin{array}{l}\text { Happened } \\
\text { to me }\end{array}$ & $\begin{array}{l}\text { Witnessed } \\
\text { it }\end{array}$ & $\begin{array}{l}\text { Learned } \\
\text { about it }\end{array}$ & $\begin{array}{l}\text { Part of my } \\
\text { job }\end{array}$ & Not sure & $\begin{array}{l}\text { Does not } \\
\text { apply }\end{array}$ \\
\hline
\end{tabular}

1. Natural disaster (for example, flood, hurricane, tornado, earthquake)

2. Fire or explosion

3. Transportation accident (for example, car accident, boat accident, train wreck, plane crash)

4. Serious accident at work, home, or during recreational activity

5. Exposure to toxic substance (for example dangerous chemicals, radiation)

6. Physical assault (not part of bullying, for example, being attacked, hit, slapped, kicked, beaten up)

7. Assault with a weapon (for example, being shot, stabbed, threatened with a knife, gun, bomb)

8. Sexual assault (rape, attempted rape, made to perform any type of sexual act through force or threat of harm)

9. Other unwanted or uncomfortable sexual experience

10. Combat or exposure to war-zone (in the military or as a civilian)

11. Captivity (for example, being kidnapped, abducted, held hostage, prisoner of war)

12. Life-threatening illness or injury

13. Severe human suffering

14. Sudden violent death (for example, homicide, suicide)

15. Sudden accidental death 
16. Serious injury, harm, or death you caused to someone else

17. Bullying victimization (for example, being threatened, stolen from, had rumours spread about you, had nasty things spread about you on the internet) 
Have you had thoughts of actually hurting yourself?

4S Screening Questions

1. Have you ever attempted to harm yourself in the past?

$$
\text { NO YES }
$$

2. Have you thought about how you might actually hurt yourself?

$$
\text { NO YES } \rightarrow \text { [How? }
$$

3. There's a big difference between having a thought and acting on a thought. How likely do you think it is that you will act on these thoughts about hurting yourself or ending your life some time over the next month?
a. Not at all likely
b. Somewhat likely
c. Very likely

4. Is there anything that would prevent or keep you from harming yourself?

NO

$$
\text { YES } \rightarrow \text { [What? }
$$

\begin{tabular}{lcc}
\hline & \multicolumn{2}{c}{ Shaded ("Risk") Response } \\
\cline { 2 - 3 } Risk Category & Items 1 and 2 & Items 3 and 4 \\
\hline Minimal & Neither is shaded & Neither is shaded \\
Lower & At least 1 item is shaded & Neither is shaded \\
Higher & & At least 1 item is shaded \\
\hline
\end{tabular}




\section{Appendix G: Posttraumatic Stress Disorder Checklist for DSM-5}

\begin{tabular}{|c|c|c|c|c|c|}
\hline In the past month, how much were you bothered by: & $\begin{array}{l}\text { Not } \\
\text { at all }\end{array}$ & $\begin{array}{c}\text { A little } \\
\text { bit }\end{array}$ & Moderately & $\begin{array}{l}\text { Quite } \\
\text { a bit }\end{array}$ & Extremely \\
\hline $\begin{array}{l}\text { 1. Repeated, disturbing, and unwanted memories of the } \\
\text { stressful experience? }\end{array}$ & 0 & 1 & 2 & 3 & 4 \\
\hline 2. Repeated, disturbing dreams of the stressful experience? & 0 & 1 & 2 & 3 & 4 \\
\hline $\begin{array}{l}\text { 3. Suddenly feeling or acting as if the stressful experience were } \\
\text { actually happening again (as if you were actually back there } \\
\text { reliving it)? }\end{array}$ & 0 & 1 & 2 & 3 & 4 \\
\hline $\begin{array}{l}\text { 4. Feeling very upset when something reminded you of the } \\
\text { stressful experience? }\end{array}$ & 0 & 1 & 2 & 3 & 4 \\
\hline $\begin{array}{l}\text { 5. Having strong physical reactions when something reminded } \\
\text { you of the stressful experience (for example, heart pounding, } \\
\text { trouble breathing, sweating)? }\end{array}$ & 0 & 1 & 2 & 3 & 4 \\
\hline $\begin{array}{l}\text { 6. Avoiding memories, thoughts, or feelings related to the } \\
\text { stressful experience? }\end{array}$ & 0 & 1 & 2 & 3 & 4 \\
\hline $\begin{array}{l}\text { 7. Avoiding external reminders of the stressful experience (for } \\
\text { example, people, places, conversations, activities, objects, or } \\
\text { situations)? }\end{array}$ & 0 & 1 & 2 & 3 & 4 \\
\hline $\begin{array}{l}\text { 8. Trouble remembering important parts of the stressful } \\
\text { experience? }\end{array}$ & 0 & 1 & 2 & 3 & 4 \\
\hline $\begin{array}{l}\text { 9. Having strong negative beliefs about yourself, other people, } \\
\text { or the world (for example, having thoughts such as: I am } \\
\text { bad, there is something seriously wrong with me, no one can } \\
\text { be trusted, the world is completely dangerous)? }\end{array}$ & 0 & 1 & 2 & 3 & 4 \\
\hline $\begin{array}{l}\text { 10. Blaming yourself or someone else for the stressful } \\
\text { experience or what happened after it? }\end{array}$ & 0 & 1 & 2 & 3 & 4 \\
\hline $\begin{array}{l}\text { 11. Having strong negative feelings such as fear, horror, anger, } \\
\text { guilt, or shame? }\end{array}$ & 0 & 1 & 2 & 3 & 4 \\
\hline 12. Loss of interest in activities that you used to enjoy? & 0 & 1 & 2 & 3 & 4 \\
\hline 13. Feeling distant or cut off from other people? & 0 & 1 & 2 & 3 & 4 \\
\hline $\begin{array}{l}\text { 14. Trouble experiencing positive feelings (for example, being } \\
\text { unable to feel happiness or have loving feelings for people } \\
\text { close to you)? }\end{array}$ & 0 & 1 & 2 & 3 & 4 \\
\hline 15. Irritable behavior, angry outbursts, or acting aggressively? & 0 & 1 & 2 & 3 & 4 \\
\hline $\begin{array}{l}\text { 16. Taking too many risks or doing things that could cause you } \\
\text { harm? }\end{array}$ & 0 & 1 & 2 & 3 & 4 \\
\hline 17. Being "superalert" or watchful or on guard? & 0 & 1 & 2 & 3 & 4 \\
\hline 18. Feeling jumpy or easily startled? & 0 & 1 & 2 & 3 & 4 \\
\hline 19. Having difficulty concentrating? & 0 & 1 & 2 & 3 & 4 \\
\hline 20. Trouble falling or staying asleep? & 0 & 1 & 2 & 3 & 4 \\
\hline
\end{tabular}


Appendix H: The Posttraumatic Cognitions Inventory

$\begin{array}{ccccccc}1 & 2 & 3 & 4 & 5 & 6 & 7 \\ \begin{array}{c}\text { totally } \\ \text { disagree }\end{array} & \begin{array}{c}\text { disagree } \\ \text { verymuch }\end{array} & \begin{array}{c}\text { disagree } \\ \text { slightly }\end{array} & \text { neutral } & \begin{array}{c}\text { agree } \\ \text { slightly }\end{array} & \begin{array}{c}\text { agree } \\ \text { verymuch }\end{array} & \begin{array}{c}\text { totally } \\ \text { agree }\end{array}\end{array}$

1. The event happened because of the way I acted

2. I can't trust that I will do the right thing

3. I am a weak person

4. I will not be able to control my anger and will do something terrible

5. I can't deal with even the slightest upset

6. I used to be a happy person but now I am always miserable

7. People can't be trusted

8. I have to be on guard all the time

9. I feel dead inside

10. You can never know who will harm you

11. I have to be especially careful because you never know what can happen next

12. I am inadequate

13. If I think about the event, I will not be able to handle it

14. The event happened to me because of the sort of person I am

15. My reactions since the event mean that I am going crazy

16. I will never be able to feel normal emotions again

17. The world is a dangerous place

18. Somebody else would have stopped the event from happening

19. I have permanently changed for the worse 
20. I feel like an object, not like a person

21. Somebody else would not have gotten into this situation

22. I can't rely on other people

23. I feel isolated and set apart from others

24. I have no future

25. I can't stop bad things from happening to me

26. People are not what they seem

27. My life has been destroyed by the trauma

28. There is something wrong with me as a person

29. My reactions since the event show that I am a lousy coper

30. There is something about me that made the event happen

31. I feel like I don't know myself anymore

32. I can't rely on myself

33. Nothing good can happen to me anymore 
Appendix I: Social Interaction Phobia Scale

\begin{tabular}{|c|c|c|c|c|c|}
\hline & $\begin{array}{c}\text { Not at all } \\
\text { characteristic } \\
\text { of me }\end{array}$ & $\begin{array}{c}\text { A little } \\
\text { characteristic } \\
\text { of me }\end{array}$ & $\begin{array}{c}\text { Somewhat } \\
\text { characteristic } \\
\text { of me }\end{array}$ & $\begin{array}{c}\text { Very } \\
\text { characteristic } \\
\text { of me }\end{array}$ & $\begin{array}{c}\text { Entirely } \\
\text { characteristic } \\
\text { of } \mathrm{me}\end{array}$ \\
\hline $\begin{array}{l}\text { 1. When mixing socially I am } \\
\text { uncomfortable. }\end{array}$ & 0 & 1 & 2 & 3 & 4 \\
\hline $\begin{array}{l}\text { 2. I have difficulty talking with other } \\
\text { people. }\end{array}$ & 0 & 1 & 2 & 3 & 4 \\
\hline $\begin{array}{l}\text { 3. I find myself worrying that I won't } \\
\text { know what to say in social } \\
\text { situations. }\end{array}$ & 0 & 1 & 2 & 3 & 4 \\
\hline $\begin{array}{l}\text { 4. I am nervous mixing with people I } \\
\text { don't know well. }\end{array}$ & 0 & 1 & 2 & 3 & 4 \\
\hline 5. I am tense mixing in a group. & 0 & 1 & 2 & 3 & 4 \\
\hline $\begin{array}{l}\text { 6. I get nervous that people are staring } \\
\text { at me as I walk down the street. }\end{array}$ & 0 & 1 & 2 & 3 & 4 \\
\hline $\begin{array}{l}\text { 7. I feel self-conscious if I have to } \\
\text { enter a room where others are } \\
\text { already seated. }\end{array}$ & 0 & 1 & 2 & 3 & 4 \\
\hline $\begin{array}{l}\text { 8. I would get tense if I had to sit } \\
\text { facing other people on a bus or a } \\
\text { train. }\end{array}$ & 0 & 1 & 2 & 3 & 4 \\
\hline $\begin{array}{l}\text { 9. I am worried people will think my } \\
\text { behavior odd. }\end{array}$ & 0 & 1 & 2 & 3 & 4 \\
\hline $\begin{array}{l}\text { 10. I would get tense if I had to carry a } \\
\text { tray across a crowded cafeteria. }\end{array}$ & 0 & 1 & 2 & 3 & 4 \\
\hline $\begin{array}{l}\text { 11. I worry l'll lose control of myself in } \\
\text { front of other people. }\end{array}$ & 0 & 1 & 2 & 3 & 4 \\
\hline $\begin{array}{l}\text { 12. I worry I might do something to } \\
\text { attract the attention of others. }\end{array}$ & 0 & 1 & 2 & 3 & 4 \\
\hline $\begin{array}{l}\text { 13. When in an elevator I am tense if } \\
\text { people look at me. }\end{array}$ & 0 & 1 & 2 & 3 & 4 \\
\hline $\begin{array}{l}\text { 14. I can feel conspicuous standing in a } \\
\text { queue. }\end{array}$ & 0 & 1 & 2 & 3 & 4 \\
\hline
\end{tabular}


Please read each statement and circle a number $0,1,2$ or 3 which indicates how much the statement applied to you over the past week. There are no right or wrong answers. Do not spend too much time on any statement.

The rating scale is as follows:

0 Did not apply to me at all

1 Applied to me to some degree, or some of the time

2 Applied to me to a considerable degree, or a good part of time

3 Applied to me very much, or most of the time

\begin{tabular}{|c|c|c|c|c|}
\hline 1 & I found it hard to wind down & 0 & 1 & 2 \\
\hline 2 & I was aware of dryness of my mouth & 0 & 1 & 2 \\
\hline 3 & I couldn't seem to experience any positive feeling at all & 0 & 1 & 2 \\
\hline 4 & $\begin{array}{l}\text { I experienced breathing difficulty (eg, excessively rapid breathing, } \\
\text { breathlessness in the absence of physical exertion) }\end{array}$ & 0 & 1 & 2 \\
\hline 5 & I found it difficult to work up the initiative to do things & 0 & 1 & 2 \\
\hline 6 & I tended to over-react to situations & 0 & 1 & 2 \\
\hline 7 & I experienced trembling (eg, in the hands) & 0 & 1 & 2 \\
\hline 8 & I felt that I was using a lot of nervous energy & 0 & 1 & 2 \\
\hline 9 & $\begin{array}{l}\text { I was worried about situations in which I might panic and make } \\
\text { a fool of myself }\end{array}$ & 0 & 1 & 2 \\
\hline 10 & I felt that I had nothing to look forward to & 0 & 1 & 2 \\
\hline 11 & I found myself getting agitated & 0 & 1 & 2 \\
\hline 12 & I found it difficult to relax & 0 & 1 & 2 \\
\hline 13 & I felt down-hearted and blue & 0 & 1 & 2 \\
\hline 14 & $\begin{array}{l}\text { I was intolerant of anything that kept me from getting on with } \\
\text { what I was doing }\end{array}$ & 0 & 1 & 2 \\
\hline 15 & I felt I was close to panic & 0 & 1 & 2 \\
\hline 16 & I was unable to become enthusiastic about anything & 0 & 1 & 2 \\
\hline 17 & I felt I wasn't worth much as a person & 0 & 1 & 2 \\
\hline 18 & I felt that I was rather touchy & 0 & 1 & 2 \\
\hline 19 & $\begin{array}{l}\text { I was aware of the action of my heart in the absence of physical } \\
\text { exertion (eg, sense of heart rate increase, heart missing a beat) }\end{array}$ & 0 & 1 & 2 \\
\hline 20 & I felt scared without any good reason & 0 & 1 & 2 \\
\hline 21 & I felt that life was meaningless & 0 & 1 & 2 \\
\hline
\end{tabular}


Appendix K; Dimensions of Anger Reactions-5 Scale

Thinking over the past 4 weeks, circle the number under the option that best described the amount of time you felt that way

\begin{tabular}{|c|c|c|c|c|c|}
\hline & $\begin{array}{l}\text { None or } \\
\text { almost none of } \\
\text { the time }\end{array}$ & $\begin{array}{l}\text { A little } \\
\text { of the } \\
\text { time }\end{array}$ & $\begin{array}{l}\text { Some of } \\
\text { the time }\end{array}$ & $\begin{array}{l}\text { Most of } \\
\text { the time }\end{array}$ & $\begin{array}{l}\text { All or almost } \\
\text { all of the } \\
\text { time }\end{array}$ \\
\hline $\begin{array}{l}\text { 1. I found myself getting angry at } \\
\text { people or situations }\end{array}$ & 1 & 2 & 3 & 4 & 5 \\
\hline $\begin{array}{l}\text { 2. When I got angry, I got really } \\
\text { mad }\end{array}$ & 1 & 2 & 3 & 4 & 5 \\
\hline $\begin{array}{l}\text { 3. When I got angry, I stayed } \\
\text { angry }\end{array}$ & 1 & 2 & 3 & 4 & 5 \\
\hline $\begin{array}{l}\text { 4. When I got angry at someone, } \\
\text { I wanted to hit them }\end{array}$ & 1 & 2 & 3 & 4 & 5 \\
\hline $\begin{array}{l}\text { 5. My anger prevented me from } \\
\text { getting along with people as } \\
\text { well as I would have like to }\end{array}$ & 1 & 2 & 3 & 4 & 5 \\
\hline
\end{tabular}


Appendix L: Distress Tolerance Scale

Think of times you feel distressed or upset. Circle the item that best describes your beliefs about feeling distressed or upset. Please answer regarding your feelings of distress 'in general', that is, on the average.

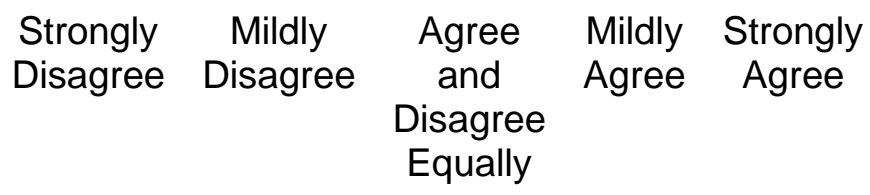
1. Feeling distressed or upset is
1
2
3
4
5 unbearable to me.

$\begin{aligned} & \text { 2. When I feel distressed or upset, } \\ & \text { all I can think about is how bad I } \\ & \text { feel. }\end{aligned}$
$\begin{aligned} & \text { 3. I can't handle feeling distressed } \\ & \text { or upset. }\end{aligned}$

$\begin{array}{llllll}\begin{array}{l}\text { 4. My feelings of distress are so } \\ \text { intense that they completely take } \\ \text { over. }\end{array} & 1 & 2 & 3 & 4 & 5 \\ \begin{array}{l}\text { 5. There's nothing worse than } \\ \text { feeling distressed or upset. }\end{array} & 1 & 2 & 3 & 4 & 5\end{array}$

$\begin{array}{llllll}\begin{array}{l}\text { 6. I can tolerate being distressed or } \\ \text { upset as well as most people. }\end{array} & 1 & 2 & 3 & 4 & 5 \\ \begin{array}{l}\text { 7. My feelings of distress or being } \\ \text { upset are not acceptable }\end{array} & 1 & 2 & 3 & 4 & 5\end{array}$

$\begin{array}{llllll}\begin{array}{l}\text { 8. I'll do anything to avoid feeling } \\ \text { distressed or upset. }\end{array} & 1 & 2 & 3 & 4 & 5 \\ \begin{array}{l}\text { 9. Other people seem to be able to } \\ \text { tolerate feeling distressed or upset } \\ \text { better than I can. }\end{array} & 1 & 2 & 3 & 4 & 5\end{array}$

$\begin{array}{llllll}\begin{array}{l}\text { 10. Being distressed or upset is } \\ \text { always a major ordeal for me. }\end{array} & 1 & 2 & 3 & 4 & 5 \\ \begin{array}{l}\text { 11. I am ashamed of myself when I } \\ \text { feel distressed or upset. }\end{array} & 1 & 2 & 3 & 4 & 5 \\ \begin{array}{l}\text { 12. My feelings of distress or being } \\ \text { upset scare me. }\end{array} & 1 & 2 & 3 & 4 & 5 \\ \begin{array}{l}\text { 13. I'll do anything to stop feeling } \\ \text { distressed or upset. }\end{array} & 1 & 2 & 3 & 4 & 5\end{array}$




$\begin{aligned} & \text { 14. When I feel distressed or upset, } \\ & \text { I must do something about it } \\ & \text { immediately. }\end{aligned}$
$\begin{aligned} & \text { 15. When I feel distressed or upset, } \\ & \text { I cannot help but concentrate on } \\ & \text { how bad the distress actually feels. }\end{aligned}$


Appendix M: Anxiety Sensitivity Index-3

Please select the number that best describes how much you agree with each statement. If any items concern something that you have never experienced (e.g., fainting in public), then answer on the basis of how you think you might feel if you had such an experience. Otherwise, answer all items on the basis of your own experience.

$\begin{array}{ccccc}\begin{array}{c}\text { Agree } \\ \text { very } \\ \text { little }\end{array} & \begin{array}{c}\text { Agree } \\ \text { a little }\end{array} & \begin{array}{c}\text { Somewh } \\ \text { at agree }\end{array} & \begin{array}{c}\text { Agree } \\ \text { a lot }\end{array} & \begin{array}{c}\text { Agree } \\ \text { very } \\ \text { much }\end{array} \\ 0 & 1 & 2 & 3 & 4\end{array}$

1. It is important to me not to appear nervous.

2. When I cannot keep my mind on a task, I

0

1

2

3

4 worry that I may be going crazy.

3. It scares me when my heart beats rapidly.

$\begin{array}{lllll}0 & 1 & 2 & 3 & 4\end{array}$

4. When my stomach is upset, I worry that I

0 1 2 34 might be seriously ill.

5. It scares me when I am unable to keep my 0 mind on a task.

6. When I tremble in the presence of others,

0 fear what people might think of me.

7. When my chest feels tight, I get scared that I

0

1

2

2

3

4 won't be able to breathe properly.

8. When I feel pain in my chest, I worry that I'm

0

1

2

3

4 going to have a heart attack.

9. I worry that other people will notice my

$0 \quad 1$

2

3

4 anxiety.

10. When I feel "spacey" or spaced out I worry

0 1 2 3 4 that I may be mentally ill.

$\begin{array}{lllll}0 & 1 & 2 & 3 & 4\end{array}$

11. It scares me when I blush in front of people.

12. When I notice my heart skipping a beat, I worry that there is something seriously wrong with me.

13. When I begin to sweat in a social situation, I 0 0 2 3 4 fear people will think negatively of me.

14. When my thoughts seem to speed up, I worry 0 1 2 3 4 that I might be going crazy. 
15. When my throat feels tight, I worry that I could

0

1

2

3

4 choke to death.

16. When I have trouble thinking clearly, I worry that there is something wrong with me.

17. I think it would be horrible for me to faint in

0

0

1

2

3

4 public.

18. When my mind goes blank, I worry there is

0

1

2

3

4 something terribly wrong with me. 
Appendix N: Intolerance of Uncertainty Scale, Short Form

Please select the number that best corresponds to how much you agree with each item.

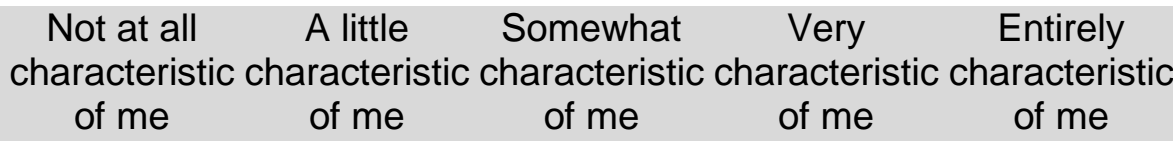

$\begin{array}{llllll}\text { 1. Unforeseen events } & 1 & 2 & 3 & 4 & 5\end{array}$
upset me greatly.

2. It frustrates me not having all the

1

23

4

information I need.

3. Uncertainty keeps me from living a full life.

4. One should always look ahead so as to avoid

12

23

4

5

surprises.

5. A small unforeseen event can spoil everything, even with 1

2

3

4

5 the best of planning.

6. When it's time to act, 1 2

3

4

5 uncertainty paralyses me.

7. When I am uncertain I can't function very well.

8. I always want to know what the future has in store for me.

9. I can't stand being taken by surprise.

10. The smallest doubt can stop me from acting.

11. I should be able to organize everything in

1

2

2

2

2

2

1

1 advance.

12. I must get away from all uncertain situations.

5

5

5

5


Appendix O: The Centrality of Event Scale

Please think back upon the bullying experience and answer the following questions in an honest and sincere way, by circling a number from 1 to 5.

\begin{tabular}{|c|c|c|c|c|c|}
\hline & $\begin{array}{c}\text { Totally } \\
\text { disagre } \\
\text { e }\end{array}$ & & & & $\begin{array}{l}\text { Totally } \\
\text { agree }\end{array}$ \\
\hline $\begin{array}{l}\text { 1. I feel that this event has become part of my } \\
\text { identity }\end{array}$ & 1 & 2 & 3 & 4 & 5 \\
\hline $\begin{array}{l}\text { 2. This event has become a reference point for } \\
\text { the way I understand myself and the world }\end{array}$ & 1 & 2 & 3 & 4 & 5 \\
\hline $\begin{array}{l}\text { 3. I feel that this event has become a central } \\
\text { part of my life story }\end{array}$ & 1 & 2 & 3 & 4 & 5 \\
\hline $\begin{array}{l}\text { 4. This event has colored the way I think and } \\
\text { feel about other experiences }\end{array}$ & 1 & 2 & 3 & 4 & 5 \\
\hline 5. This event has permanently changed my life & 1 & 2 & 3 & 4 & 5 \\
\hline $\begin{array}{l}\text { 6. I often think about the effects that this event } \\
\text { will have on my future }\end{array}$ & 1 & 2 & 3 & 4 & 5 \\
\hline 7. This event was a turning point in my life & 1 & 2 & 3 & 4 & 5 \\
\hline
\end{tabular}


Appendix P: Cognitive Emotion Regulation Questionnaire- Short

\begin{tabular}{|c|c|c|c|c|c|}
\hline & $\begin{array}{l}\text { Almost } \\
\text { never }\end{array}$ & & & & $\begin{array}{l}\text { Almost } \\
\text { always }\end{array}$ \\
\hline $\begin{array}{l}\text { 1. I feel that I am the one who is responsible } \\
\text { for what has happened }\end{array}$ & 1 & 2 & 3 & 4 & 5 \\
\hline $\begin{array}{l}\text { 2. I think that basically the cause must lie } \\
\text { within myself }\end{array}$ & 1 & 2 & 3 & 4 & 5 \\
\hline $\begin{array}{l}\text { 3. I think that I have to accept that this has } \\
\text { happened }\end{array}$ & 1 & 2 & 3 & 4 & 5 \\
\hline 4. I think that I have to accept the situation & 1 & 2 & 3 & 4 & 5 \\
\hline $\begin{array}{l}\text { 5. I often think about how I feel about what I } \\
\text { have experienced }\end{array}$ & 1 & 2 & 3 & 4 & 5 \\
\hline $\begin{array}{l}\text { 6. I am preoccupied with what I think and feel } \\
\text { about what I have experienced }\end{array}$ & 1 & 2 & 3 & 4 & 5 \\
\hline $\begin{array}{l}\text { 7. I think of pleasant things that have nothing } \\
\text { to do with it }\end{array}$ & 1 & 2 & 3 & 4 & 5 \\
\hline $\begin{array}{l}\text { 8. I think of something nice instead of what has } \\
\text { happened }\end{array}$ & 1 & 2 & 3 & 4 & 5 \\
\hline 9. I think about how to change the situation & 1 & 2 & 3 & 4 & 5 \\
\hline 10. I think about a plan of what I can do best & 1 & 2 & 3 & 4 & 5 \\
\hline $\begin{array}{l}\text { 11. I think I can learn something from the } \\
\text { situation }\end{array}$ & 1 & 2 & 3 & 4 & 5 \\
\hline $\begin{array}{l}\text { 12. I think that I can become a stronger person } \\
\text { as a result of what has happened }\end{array}$ & 1 & 2 & 3 & 4 & 5 \\
\hline $\begin{array}{l}\text { 13. I think that it hasn't been too bad compared } \\
\text { to other things }\end{array}$ & 1 & 2 & 3 & 4 & 5 \\
\hline $\begin{array}{l}\text { 14. I tell myself that there are worse things in } \\
\text { life }\end{array}$ & 1 & 2 & 3 & 4 & 5 \\
\hline $\begin{array}{l}\text { 15. I keep thinking about how terrible it is what } \\
\text { I have experienced }\end{array}$ & 1 & 2 & 3 & 4 & 5 \\
\hline $\begin{array}{l}\text { 16. I continually think how horrible the situation } \\
\text { has been }\end{array}$ & 1 & 2 & 3 & 4 & 5 \\
\hline $\begin{array}{l}17 . \text { I feel that others are responsible for what } \\
\text { happened }\end{array}$ & 1 & 2 & 3 & 4 & 5 \\
\hline $\begin{array}{l}\text { 18. I feel that basically the cause lies with } \\
\text { others }\end{array}$ & 1 & 2 & 3 & 4 & 5 \\
\hline
\end{tabular}


Appendix Q: The Brief Resilience Scale

1. Please circle the number that best corresponds to how much you agree with each item.

\begin{tabular}{|lccccc|}
\hline & $\begin{array}{c}\text { Strongly } \\
\text { disagree }\end{array}$ & Disagree & Neutral & Agree & $\begin{array}{c}\text { Strongly } \\
\text { agree }\end{array}$ \\
\hline $\begin{array}{l}\text { 1. I tend to bounce back quickly after } \\
\text { hard times }\end{array}$ & 1 & 2 & 3 & 4 & 5 \\
$\begin{array}{l}\text { 2. I have a hard time making it through } \\
\text { stressful events }\end{array}$ & 1 & 2 & 3 & 4 & 5 \\
$\begin{array}{l}\text { 3. It does not take me long to recover } \\
\text { from a stressful event }\end{array}$ & 1 & 2 & 3 & 4 & 5 \\
$\begin{array}{l}\text { 4. It is hard for me to snap back when } \\
\text { something bad happens }\end{array}$ & 1 & 2 & 3 & 4 & 5 \\
$\begin{array}{l}\text { 5. I usually come through difficult times } \\
\text { with little trouble }\end{array}$ & 1 & 2 & 3 & 4 & 5 \\
6. I tend to take a long time to get over \\
$\quad$ set-backs in my life
\end{tabular}


Appendix R: The Multidimensional Scale of Perceived Social Support

Circle the "1" if you Very Strongly Disagree

Circle the " 2 " if you Strongly Disagree

Circle the "3" if you Mildly Disagree

Circle the "4" if you are Neutral

Circle the "5" if you Mildly Agree

Circle the "6" if you Strongly Disagree

Circle the "7" if you Very Strongly Disagree

$\begin{array}{llllllllll}1 \text { There is a special person who is around when I } & 1 & 2 & 3 & 4 & 5 & 6 & 7\end{array}$ am in need
2. There is a special person with whom I can share
1
$2 \quad 3 \quad 4$
5
6
7

my joys and sorrows

$\begin{array}{lllllll}1 & 2 & 3 & 4 & 5 & 6 & 7\end{array}$

3 My family really tries to help me

\begin{tabular}{|c|c|c|c|c|}
\hline $\begin{array}{l}4 \text { I get the emotional help and support I need from } \\
\text { my family }\end{array}$ & 1 & 2 & 3 & \\
\hline
\end{tabular}

5 I have a special person who is a real source of

$\begin{array}{lllllll}1 & 2 & 3 & 4 & 5 & 6 & 7\end{array}$
comfort to me

6 My friends really try to help me

$\begin{array}{lllllll}1 & 2 & 3 & 4 & 5 & 6 & 7\end{array}$

7 I can count on my friends when things go wrong

$\begin{array}{lllllll}1 & 2 & 3 & 4 & 5 & 6 & 7\end{array}$

8 I can talk about my problems with my family

$\begin{array}{lllllll}1 & 2 & 3 & 4 & 5 & 6 & 7\end{array}$

9 I have friends with whom I can share my joys and
sorrows

10 There is a special person in my life who cares

$1 \quad 2 \quad 3$
about my feelings

11 My family is willing to help me make decisions

$\begin{array}{lllllll}1 & 2 & 3 & 4 & 5 & 6 & 7 \\ 1 & 2 & 3 & 4 & 5 & 6 & 7\end{array}$

12 I can talk about my problems with my friends 
Appendix S: CPT for Social Trauma

\begin{tabular}{|c|c|c|c|}
\hline $\begin{array}{c}\text { Session } \\
\text { Number }\end{array}$ & Session Theme & Session Content & Homework \\
\hline 1 & $\begin{array}{l}\text { Social trauma and } \\
\text { treatment rational }\end{array}$ & $\begin{aligned} & \text { Psychoeducation: } \\
& \text { - } \text { Cognitions, behavior, } \\
& \text { emotions } \\
& \text { - } \text { Social trauma } \\
& \text { - } \text { PTSD } \\
& \text { - } \text { Stuck points } \\
& \text { - } \text { Treatment rational }\end{aligned}$ & $\begin{array}{l}\text { Write an impact statement } \\
\text { outlining the meaning of the } \\
\text { bullying experience }\end{array}$ \\
\hline 2 & Stuck points & $\begin{array}{l}\text { Stuck points: assimilation, } \\
\text { overaccommodation } \\
\text { - Identify stuck points in } \\
\text { impact statement } \\
\text { - } \text { ABC sheets }\end{array}$ & Complete an ACC sheet \\
\hline 3 & $\begin{array}{l}\text { Difference between } \\
\text { cognitions and } \\
\text { emotions }\end{array}$ & $\begin{array}{l}\text { Review homework } \\
\text { Thought challenging and Socratic } \\
\text { questions }\end{array}$ & $\begin{array}{l}\text { Write a trauma account of most } \\
\text { distressing bullying experience } \\
\text { and complete daily } \mathrm{ABC} \text { sheets }\end{array}$ \\
\hline 4 & Trauma account & $\begin{array}{l}\text { Re-write trauma account with an } \\
\text { emphasis of incorporating } \\
\text { emotions. } \\
\begin{aligned}- & \text { Read it aloud } \\
- & \text { Identify stuck points } \\
- & \text { Consider contextual } \\
& \text { factors }\end{aligned}\end{array}$ & $\begin{array}{l}\text { Re-write the trauma account and } \\
\text { complete daily } \mathrm{ABC} \text { sheets }\end{array}$ \\
\hline 5 & $\begin{array}{l}\text { Trauma account with } \\
\text { stuck points }\end{array}$ & $\begin{array}{l}\text { Read trauma account aloud } \\
\text { Challenging questioning sheet }\end{array}$ & $\begin{array}{l}\text { Challenge at least one stuck } \\
\text { point per day }\end{array}$ \\
\hline 6 & $\begin{array}{l}\text { Stuck point } \\
\text { challenging }\end{array}$ & $\begin{array}{l}\text { Review homework } \\
\text { Challenge stuck points } \\
\text { Faulty thinking patterns } \\
\text { introduced }\end{array}$ & Record faulty thinking patterns \\
\hline 7 & $\begin{array}{l}\text { Dysfunctional } \\
\text { beliefs: safety }\end{array}$ & $\begin{array}{l}\text { Themes selected on individual } \\
\text { basis }\end{array}$ & Challenging belief worksheet \\
\hline 8 & $\begin{array}{l}\text { Dysfunctional } \\
\text { beliefs: trust }\end{array}$ & $\begin{array}{l}\text { Themes selected on individual } \\
\text { basis }\end{array}$ & Challenging belief worksheet \\
\hline 9 & $\begin{array}{l}\text { Dysfunctional } \\
\text { beliefs: } \\
\text { power/control }\end{array}$ & $\begin{array}{l}\text { Themes selected on individual } \\
\text { basis }\end{array}$ & Challenging belief worksheet \\
\hline 10 & $\begin{array}{l}\text { Dysfunctional } \\
\text { beliefs: intimacy }\end{array}$ & $\begin{array}{l}\text { Themes selected on individual } \\
\text { basis }\end{array}$ & Challenging belief worksheet \\
\hline 11 & $\begin{array}{l}\text { Dysfunctional } \\
\text { beliefs: esteem }\end{array}$ & $\begin{array}{l}\text { Themes selected on individual } \\
\text { basis }\end{array}$ & Challenging belief worksheet \\
\hline 12 & $\begin{array}{l}\text { Treatment } \\
\text { termination }\end{array}$ & $\begin{array}{l}\text { Review and reflection of } \\
\text { treatment progress. } \\
\text { Relapse prevention }\end{array}$ & \\
\hline
\end{tabular}


Appendix T: SM for Social Trauma

\begin{tabular}{|c|c|c|c|}
\hline $\begin{array}{c}\text { Session } \\
\text { Number }\end{array}$ & Session Theme & Session Content & Homework \\
\hline 1 & $\begin{array}{l}\text { Introduction and } \\
\text { physiology of stress }\end{array}$ & $\begin{array}{l}\text { Treatment rational } \\
\text { Nature and physiology of stress: } \\
\text { - } \\
\text { The fight, flight, freeze } \\
\text { system and bullying } \\
\text { - }\end{array}$ & $\begin{array}{l}\text { Monitor and record stressful } \\
\text { situations and physical stress } \\
\text { responses }\end{array}$ \\
\hline 2 & $\begin{array}{l}\text { Diaphragmatic } \\
\text { Breathing }\end{array}$ & $\begin{array}{l}\text { Breathing to relax } \\
\text { Diaphragmatic breathing } \\
\text { exercises }\end{array}$ & $\begin{array}{l}\text { Daily diaphragmatic breathing } \\
\text { exercises for } 10-15 \text { minutes }\end{array}$ \\
\hline 3 & Guided imagery & Guided imagery for relaxation & $\begin{array}{l}\text { Daily imagery and } \\
\text { diaphragmatic breathing } \\
\text { exercises for } 10-15 \text { minutes }\end{array}$ \\
\hline 4 & Soothing activities & $\begin{array}{l}\text { Engagement of the five senses for } \\
\text { relaxation and soothing }\end{array}$ & $\begin{array}{l}\text { One soothing activity per day. } \\
\text { Daily diaphragmatic breathing } \\
\text { exercises for } 10-15 \text { minutes }\end{array}$ \\
\hline 5 & $\begin{array}{l}\text { Progressive muscle } \\
\text { relaxation (PMR) }\end{array}$ & $\begin{array}{l}\text { Psychoeducation and PMR } \\
\text { exercise }\end{array}$ & $\begin{array}{l}\text { Daily PMR exercises for } 10 \\
\text { minutes. }\end{array}$ \\
\hline 6 & $\begin{array}{l}\text { Progressive muscle } \\
\text { relaxation (PMR) }\end{array}$ & $\begin{array}{l}\text { Psychoeducation and PMR } \\
\text { exercise }\end{array}$ & $\begin{array}{l}\text { Daily PMR exercises for } 10 \\
\text { minutes }\end{array}$ \\
\hline 7 & $\begin{array}{l}\text { Problem-focused and } \\
\text { emotion focused } \\
\text { coping }\end{array}$ & $\begin{array}{l}\text { Introducing flexibility in stress } \\
\text { management }\end{array}$ & $\begin{array}{l}\text { Monitor stress coping } \\
\text { strategies }\end{array}$ \\
\hline 8 & $\begin{array}{l}\text { Stress management } \\
\text { in daily life }\end{array}$ & $\begin{array}{l}\text { Incorporating stress reduction } \\
\text { strategies into daily life }\end{array}$ & $\begin{array}{l}\text { Daily PMR exercises for } 10 \\
\text { minutes }\end{array}$ \\
\hline 9 & $\begin{array}{l}\text { Stress management } \\
\text { in daily life }\end{array}$ & $\begin{array}{l}\text { Incorporating stress reduction } \\
\text { strategies into daily life }\end{array}$ & $\begin{array}{l}\text { Activity selected based on } \\
\text { participant preference }\end{array}$ \\
\hline 10 & $\begin{array}{l}\text { Stress management } \\
\text { in daily life }\end{array}$ & $\begin{array}{l}\text { Incorporating stress reduction } \\
\text { strategies into daily life }\end{array}$ & $\begin{array}{l}\text { Activity selected based on } \\
\text { participant preference }\end{array}$ \\
\hline 11 & $\begin{array}{l}\text { Stress management } \\
\text { in daily life }\end{array}$ & $\begin{array}{l}\text { Incorporating stress reduction } \\
\text { strategies into daily life }\end{array}$ & $\begin{array}{l}\text { Activity selected based on } \\
\text { participant preference }\end{array}$ \\
\hline 12 & $\begin{array}{l}\text { Treatment } \\
\text { termination }\end{array}$ & $\begin{array}{l}\text { Review and reflection of } \\
\text { treatment progress. } \\
\text { Relapse prevention }\end{array}$ & \\
\hline
\end{tabular}




\section{Appendix U: CPT Content Survey}

Please consider each statement and use the scale provided to rate it.

1. How many online lessons of the cognitive processing therapy program did you complete?

\section{$\begin{array}{llllllllllll}1 & 2 & 3 & 4 & 5 & 6 & 7 & 8 & 9 & 10 & 11 & 12\end{array}$}

(qualtrics logic) If only 1 session:

1a: The reason I did not complete more than one online lessons (please choose one or more)

1. I did not find them helpful

2. The online format of the program did not suit me (I prefer to see someone in person)

3. I was enrolled in another type of treatment

4. I was too busy with work/other things in my life

5. I found it too time consuming

6. Other (please describe)

(then Thank you for participating in our program- end of survey)

(qualtrics logic) If completed more than 1 session:

2. Overall, I found the cognitive processing therapy helpful:
a. Strongly agree
b. Agree
c. Somewhat agree
d. Neither agree nor disagree
e. Somewhat disagree
f. Disagree
g. Strongly disagree

3. I found the following helpful (please choose one or more):

a. Impact statement (in the first two sessions where I wrote about how bullying has impacted me) 
b. Written trauma account (in sessions three and four where I identified my worst bullying experience and wrote it down in detail)

c. Common patterns of problematic thinking (introduced in session six. For example: mindreading, jumping to conclusion, emotional reasoning)

d. ACB worksheets (where I learned about how thoughts affect emotions by writing down: activating event, belief/stuck point and consequence)

e. Challenging beliefs worksheet (introduced in session five, where I asked myself questions like "Is my stuck point based on habit or fact, and in what ways is my stuck point based on feelings rather than facts")

f. Learning about safety issues (session seven. For example stuck points like "Other people are always going to hurt me")

g. Learning about trust issues (session eight. For example stuck points like "I am unable to make good decisions/I can never trust others")

h. Learning about power/control issues (session nine. For example "I need to be in total control")

i. Learning about esteem issues (session 10. For example: "There is something fundamentally wrong with me")

j. Learning about intimacy issues (session 11. For example: "No relationship ever works out for me")

k. None of the above (please describe)

4. The length of this treatment program was:
a. Too long
b. Slightly too long
c. Adequate
d. Slightly too short
e. Too short
f. Unsure

5. I found communication with my therapist helpful:
a. Strongly agree
b. Agree
c. Somewhat agree
d. Neither agree nor disagree
e. Somewhat disagree
f. Disagree
g. Strongly disagree

6. I worked hard in this treatment

a. Definitely true 

b. Probably true
c. Neither true nor false
d. Probably false
e. Definitely false

7. I practiced the skills taught in the treatment
a. Definitely true
b. Probably true
c. Neither true nor false
d. Probably false
e. Definitely false

8. I used the skills taught in the treatment in my daily life
a. A great deal
b. A lot
c. A moderate amount
d. A little
e. None at all

9. My beliefs about myself/other people changed in helpful ways from this treatment
a. Strongly agree
b. Agree
c. Somewhat agree
d. Neither agree nor disagree
e. Somewhat disagree
f. Disagree
g. Strongly disagree

10. My beliefs about my bullying experience/s changed in helpful ways from this treatment
a. Strongly agree
b. Agree
c. Somewhat agree
d. Neither agree nor disagree
e. Somewhat disagree
f. Disagree
g. Strongly disagree

11. I would recommend this program to people 

a. Strongly agree
b. Agree
c. Somewhat agree
d. Neither agree nor disagree
e. Somewhat disagree
f. Disagree
g. Strongly disagree

12. Other comments? (optional) 


\section{Appendix V: SM Content Survey}

Please consider each statement and use the scale to rate it.

1. How many online lessons of the stress management program did you complete?

$$
\begin{array}{llllllllllll}
1 & 2 & 3 & 4 & 5 & 6 & 7 & 8 & 9 & 10 & 11 & 12
\end{array}
$$

(qualtrics logic) If only 1 session:

1a: The reason I did not complete more than one online lessons (please choose one or more)

a. I did not find them helpful

b. The online format of the program did not suit me (I prefer to see someone in person)

c. I was enrolled in another type of treatment

d. I was too busy with work/other things in my life

e. I found it too time consuming

f. Other (please describe)

(then Thank you for participating in our program- end of survey)

(qualtrics logic) If completed more than 1 session:

2. Overall, I found the stress management program helpful
a. Strongly agree
b. Agree
c. Somewhat agree
d. Neither agree nor disagree
e. Somewhat disagree
f. Disagree
g. Strongly disagree

3. I found the following helpful (please choose one or more):

a. Identifying and recording stressful situation and my reactions (in the first session)

b. Diaphragmatic Breathing (session two; where you were asked to practice breathing with your diaphragmatic muscle, "belly breathing")

c. Guided Imagery for Relaxation (session three, where you brought your attention to a calming scenario in your mind and imaged what you would hear, smell and feel in that scenario) 
d. Five Senses Practice (session four; where you directed your attention to sensations in your body, to hearing, tasting, smelling)

e. Progressive Muscle Relaxation (in sessions five and six, where you tensed and relaxed your muscles for relaxation)

f.Every Day Strategies for Stress Reduction (in session seven, where you learned about active and passive coping)

g. Learning lifestyle choices and stress (sessions eight, nine and 10. For example how nutrition and exercise can be helpful, and about sleep hygiene)

h. The Hour-by-myself technique (session 11, where you learned about taking time away from stimulation for stress management and relaxation)

i. None of the above (please describe)

7. The length of this program was:
a. Too long
b. Slightly too long
c. Adequate
d. Slightly too short
e. Too short
f. Unsure

8. I found communication with my therapist helpful:
a. Strongly agree
b. Agree
c. Somewhat agree
d. Neither agree nor disagree
e. Somewhat disagree
f. Disagree
g. Strongly disagree

6. I worked hard in this program
a. Definitely true
b. Probably true
c. Neither true nor false
d. Probably false
e. Definitely false

7. I practiced the skills taught in the program
a. Definitely true
b. Probably true
c. Neither true nor false
d. Probably false
e. Definitely false 
8. I used the skills taught in the program in my daily life
a. A great deal
b. A lot
c. A moderate amount
d. A little
e. None at all

9. My beliefs about myself/other people changed in helpful ways from this program
a. Strongly agree
b. Agree
c. Somewhat agree
d. Neither agree nor disagree
e. Somewhat disagree
f. Disagree
g. Strongly disagree

10. My beliefs about my bullying experience/s changed in helpful ways from this program
a. Strongly agree
b. Agree
c. Somewhat agree
d. Neither agree nor disagree
e. Somewhat disagree
f. Disagree
g. Strongly disagree

11. I would recommend this program to people
a. Strongly agree
b. Agree
c. Somewhat agree
d. Neither agree nor disagree
e. Somewhat disagree
f. Disagree
g. Strongly disagree

12. Other comments? (optional) 